PM

1274

B5?

1899

SOA 

hang. 

IIA.

A 



\section{THE G,OSPEL}

ACCORDING TO

\section{SAINT LUKE}

IN

\section{HAIDA *}

Keen, g. H. tr.]

\section{LONDON :}

PRINTED FOR THE

BRITISH AND FOREIGN BIBLE SOCIETY.

1899. 


$$
\begin{gathered}
\text { PM1274 } \\
1357 \\
1899
\end{gathered}
$$

1) 1 : $\{1\} 13$

TRANSLATED BY

'THE REV. ऽ. H. KEEN,

MISAIONARY OF THE CHURCH MISSIONARY SOCIETY. 


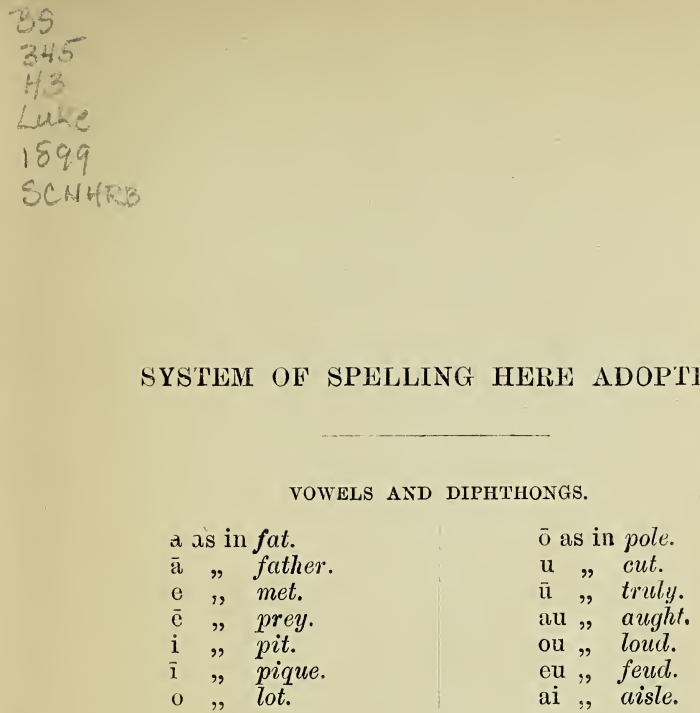

CONSONANTS.

$\mathrm{g}$ hard, as in go.

$\mathrm{j}$ as in jest.

k as ch in loch.

$s$ as in sit.

ch, chip.

w and y are used only as consonants.

lth represents a sound made by expelling the breath while the tip of the tongue touches the palate.

Hyphens are occasionally introduced to aid the reader in dividing the syllables. 



\section{GIALTHANG LA \\ S A I N T L U K E

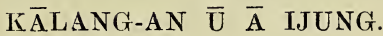

\section{Chapter 1.}

I/LIKU gin itil shu edan tl kwon kālung-ai 1 isdagudung-gun alth- $\overline{\mathrm{u}}$,

2. Kwunstie sta ga keng-gang-an isgien, Shanung-itlagadas kil un ga liplētgāgan ishin, itil ga shudaian ging-an,

3. Kwunstie sta gin wautliwon tliku edangan un yenki di ūnshidulgun alth, duman dung un kālung-ai da di ishin gudung-gun, Theophilus lādsilthīs ;

4. Gin dung tl shkotadaian yenki edan un dung īnshidai una.

5. Herod Judæa tligai un king-gāgan nūt, nung liplētga Zacharias hin kyā jjan, waigien Abijah gia haadgai alth il lthong-gwil-gangan; waigien Aaron gūjang-alung swon il ināgan Elizabeth hin il kyāgan.

6. Waigien il stung-won Shanung-itlagadas hang-gu lā-āwon, waigien Shalana gia king-gogung-ai wautliwon isgien, tliku gin il et-hul- 
gang-an ishin ging-an tlishgūda ădan il etgī$\overline{\mathrm{u}}$-gang-an.

7. Waigien gum il git-kai-ung-āwon, Elizabeth kekulaian altha, waigien il stung-won kē-ēlthāwon.

8. Waigien Shanung-itlagadas hang-gu tliku la tl ethalan ging-an nung liplëtgas gia lthong-gwilie il isdagundān,

9. Tliku ga liplètgasgai edang-an ging-an, Shalana gia temple-gai ai il kudsas gien, shkw ūnūlou il ōgulthdie lāgan.

10. Waigien shkwūnūlou tl ogulthdaiandlu, tl shkūlas wantliwon kyāgūst singlthgang gushu-gang-an.

11. Waigien Shalana gia angel-gai swon shkwūnūlou altar-gai sōolgūst giäns il. kangan.

12. Waigien Zacharias gudung-ai lthkwidaian tlistleuon la il kang-andlu-a, waigien il lthwaugalan.

13. Waigien nung angel-gas hin il shudaian, Zacharias, gum lth lthwaugang: singlthgang dung gushus Shanung-itlagadas guduns altha, waigien dung jā Elizabeth nung îlthinga dung odō sta kai-ishang, waigien John hin la dung kyādā-ashang.

14. Waigien dung gudung-ai las gien, tlika dung gwūlā-ashang; waigien tl kwon la tl kais alth gudung-ai lā-ashang.

15. Shalana hang-gu il eu-on-ses altha, wai- 
gien wine isgien gin dutga ishin gum il nilthang-ashang; waigien ou-ung dulthi sta-hun anung Hants Las alth il stāūgā-ashang.

16. Waigien Israel gitalung kwon Shalana Shanung-itlagadas gwī ung-a il tla-stīlthishang.

17. Waigien Elijah gudung-ai isgien, dugwīgai alth ishin, la kōnast il kā-ashang, tl aungalung gudung-ai gitalung gwi il tla-stilths gien, ga geujiasgai ga lasgai kutung-gai ai ishin il al-istalai una; tl haada Shalana un agung elthgīdas il tla-elthgīgai una, hin la il shudaian.

18. Gushinu adshi un di ūnshidulth-tljūashang ? di kēe-ēlths gien, di jā ishin kē-ēlths altha, waigien hin Zacharias nung angel-gas shudaian.

19. Waigien nung angel-gas hin hanglthang il shudaian, Gabriel ltha-ū ijung, Shanung-itlagadas hang-gu nung giāans-a; waigien dung $\bar{a}$ lth gushue un isgien, a gialthang lā is ishin dung ga lth shudie un di tl kil-jjun.

20. Waigien, gwokeng, dung kilgūūdeāashang, waigien kilthgulie odō dung eshgāashang, ga shantlanai gut a gin is wai ging-an etgai keu-a, time-gai elthgīsdlu tliku lth shūs ging-an etses gum dung yet-dang-gun altha, hin la il shudaian.

21. Waigien tl haadas Zacharias keu edan, 
waigien temple-gai ā il gũ-hunsgadan un 11 kuldung-āgan.

22. Waigien tlistleuon il katla-waugandlu, tla à gushue odō il eshgaian; waigien templegai à gin unkuldung-a il kang-an un tl ünshidan ; waigien tla hung-ā stlang il akaiādaian, waigien hawun il kil-gāwon.

23. Waigien waudlā, thistleuon sing gūit il lthong-gwilang-kashagan tlan èlandlu, giang nai ã il kaidan.

24. Waigien ashgai shantlanai dlā, il jā Elizabeth dulthgièlan; waigien kōng kē-tlēlth gūt ung il salgādaian,

25. Hin Shalana di isdagun, ga shantlanai ai di il keng-gundlu, tl haada shu di ëdans il gīillthdie una, waigien hin il shouon.

26. Waigien kōng kē-tlū-unalandlu, Gabriel nung angel-ga Shanung-itlagadas sta Galilee ilna-gai swon Nazareth hin kyas gu katlagan,

27. Nung dliang nung îlthing-a Joseph hin kias, David gia haadgai swon ijan, inang-kasas un il katlagan; waigien nung dlians Mary hin kiāgan.

28. Waigien la kwulth il kudsaian gien, Dung $\bar{a}$ lth kil-lāgung, yenki dung tl tloulthligulths, Shalana dung alth ijung, hin il shouon. 29. Waigien tliku il shouon alth Mary gudung-ai lthkwīd-euon-an gien, tliku adshi gushue etgai odō il kwu ùlthalan.

30. Waigien nung angel-gas hin il shudaian, 
Gum Ith lthwaugang, Mary; Shanung-itlagadas dung alth gudung-ai las altha.

31. Waigien, gwokeng, dung dulthgiēlthshang, waigien nung ilthing-a dung kai-ishang, waigien JESUS hin la dung kyādāashang.

32. Il euon-shang, waigien sha agwi Nung is Git hin il kya-ashang; waigien Shalana Shanung-itlagadas David il aung gia aulthgahung-we la ga isdāashang;

33. Waigien Jacob gia haadgai un il itlagada-swonung-ashang; waigien lagia kingdomgai gum tlan elth-swonung-ung-ashang, hin nung angel-gas Mary shudaian.

34. Gushinu adshi etishang, gum lth lāinans altha? waigien hin Mary nung angelgas shudaian.

35. Waigien nung angel-gas hin hanglthang il shudaian, Nung Hants Las dung ing-gu isishang, waigien sha āgwī Nung is gia dugwīgai dung à lthkāūjēlthashang; althji alth $\bar{u}$ nung dung kai-ises nung las isgien, Shanungitlagadas Git ishin, hin kyäashang.

36. Waigien, gwokeng, dung tou-i Elizabeth ishin kè-ëlths gien dulthgiēlgung; waigien kekula hin nung kyāgun da kōng kë-tlū-unulgung.

37. Kil wautliwon Shanung-itlagadas sta isis gum dugwīa ädan isang-ases altha, hin nung angel-gas il shudaian.

38. Shalana gitsada di ijung; tliku dung 
shus ging-an Ith akwon di un edi, waigien hin Mary shouon. Waigien nung angel-gas la sta kaidan.

39. Waigien ashgai shantlanai gut Mary giāagan gien, tliga gu tldou kwons ā ung il gūdūlthāgang-an, Judah tligai gu lana jjan ā-a ;

40. Waigien Zacharias kwulth il kudsaian gien, Elizabeth ā il kil-lāgan.

41. Waigien tlistleuon Elizabeth Mary la à kil-las gudang:-andlu, il dul à nung îkutsūs vildang-an; waigien Elizabeth Hants Las alth stahgan ;

42. Waigien kwunan il kilthgūlaian, Tl jaadas shu dung las gien, nung dung kaiases ishin lāgung.

43. Waigien gushintlou giagun Shalana-gai ou di keng-atlang?

44. Gwokeng, tlistleuon di ā dung kil-las lth gudung-gundlu, nung îkutsūs gudung-ai là alth di dul à yildung-gun.

45. Waigien nung yetdagun $\bar{u}$ lāgung; Shalana sta gin tl shudaian wai ging-an etses altha, waigien hin Elizabeth Mary shudaian.

46. Waigien Mary hin shouon, Di althandai Shalana ā kil-]āgung,

47. Waigien Shanung-itlagadas Nung alth di koguns alth di gudung-ai tlika-gūlāgun.

48. Il gitsada jadas het gudung-ai ets il kang-an altha; adshi sta gūdista kang-gai wautliwon, nung las, hin di kyādāashang. 
49. Nung Dugwia-euons di unyāgudungèlthdagun altha, waigien il kyē ù lāgung.

50. Waigien gūdista kang-gai kwon shū sta la ga ga lthwaugasgai kalthshint il gudunggung.

51. Hyang alth dugwia tla il kendaian; ga gudung-ai sha etsgai hawun gin odō kwulthilgundān il hagujang-an.

52. Ga prince-gasgai aulthgahung-we sta hēt ga ung-a il isdaian, hēt ga ga gudung-ai etsgai ishin shi ga il isdaian.

53. Ga k'wōtsgai gin lā alth il skisūdaian; waigien ga gin-lthing-asgai kēstlang-an il kilistiidan.

54. Israel gitsadang da il tlaadan, Abraham un isgien, il gitalung un ishin.

55. (Tliku itil aung-alung ga il shouon gingan) k'ung-gudung-ie ā ung-a il gūshgut-swonung-ai una, hin Mary shouon.

56. Waigien Mary kōng kē-lthōnulth dlu la alth nāgan gien, gyang nai gwī silthgang il stēlan.

57. Waigien Elizabeth kaigai dlu ga èlan; waigien nung ilthing-a il kaian.

58. Waigien la kwulthit ga näansgai isgien il tou-alung ishin Shalana la kalthshint gudungeuon-an $\bar{u}$ gudang-an; waigien la alth tlika tl gwūlāgan.

59. Waigien shin stanshang-āgandlu nung ākntsūs circumcise-die un tl istlagan ; waigien, 
Zarharias, hin la tl kyādang-kashagan, tliku il aung kyāgan ging-an-a.

60. Gāanū ; John hin tlou il kyaaashang, waigien hin il ou hanglthang shouon.

61. Gum dung tou-i swon-hun hin kyā-anggung, waigien hin la tl shudaian.

62. Waigien il aung hung-ã stlang tl akaiadaian gien, tliku il kyāgai da il gudung-ai da la alth tl kiānang-an.

63. Waigien gin kālung-ai gū da il kiānangan gien, John, hinū il kyang, hin il kālang-an. Waigien un tl kuldung-ā-ōdsou-on.

64. Waigien hawidan il helthi helthalan gien, il tong-il ishin ai otadan, waigien il gushouon gien, Shanung-itlagadas à il killāgan.

65. Waigien tla kwulthît ga nāang-an-gai wautliwon lthwaugalan; waigien Judæa tligai tldāwe kwons wautliwon ai tliku tl shouon wautliwon king-ā-koküstlūiaian.

66. Waigien ga gudang-an-gai wautliwon wau $\bar{a}$ guidanung-ēdan gien, Gushinu anung ākntsūs èdung-kasha-alth? hin tl shouon. Shalana stlai la alth ijan altha.

67. Waigien il aung Zacharias Hants Las alth stahgan gien, gin unsta il shouon, waigien hin il shouon,

68. Shalana Israel un nung Itlagadas ĩ iāgung; haadgai un ung-a il katlagan gien, tla il dahgan altha, 
69. Waigien David il gitsada gia nai à kogan nushang-wē itil un il isdaian

70. (Hetligai isīdan sta tliku prophet las helthi alth ung-a il shouou ging-an-a,)

71. Itil telg ga kensgai sta isgien, itil tloulthdā-ung-ie da ga guduńsgai wautliwon stlai sta ishin itil il kogundaian;

72. Itil aung-alung kalthshint il guduns gien, gin lā il shudaian ã ishin ung-a il gudanung-ai una ;

73. Abraham itil aung $\bar{a}$ gushou tladsga alth gin il shudaian-a,

74. Itil telg ga kensgai stlai sta itil kogunsdlu Ithwauga ädan,

75. Hēnung-a lā alth isgien, hēnung-a yā alth ishin, itil hēnung-askialthk la hang-gu lit un talung lthong-gwilie un itil ga il isdie una.

76. Nung ākntsīs, waigien dā-ū sha āgwi Nung ets gia prophetgai hin kyā-ashang; Shalana gia keu-e elthgīdie un la konast dung kāases altha ;

77. Gyang haadgai il kogundāases isgien, tl dãung-as kalthshint ishin il gudung-ases un tla dung ūnshidulthdie una,

78. Itil Itlagida kung-gudung-as alth- $\bar{u}$, sha $\bar{a} g$ wi sta shantlan otulthgwai is itil un katlāashang,

79. Alga ā isgien, kotal algagai à ga tlodas ishin $\bar{a}$ kokeshguts gien, keu dlaia ai itil alistalai una, hin Zacharias shouon. 
80. Waigien nung āḱutsūs innastlaian gien, il gudung-ai tladsgēlan, waigien Israel haade la tl kendie keu, tligunltha il ijan.

\section{Chapter 2.}

WAIGIEN althsgai shantlanai gut hēt-tligai gu tl haada is wautliwon kyē tl kālungai un Cæsar Augustus tuking-gogang-an.

2. Aū tlishinūt tl kyē tlang-a tl kālang-an Quirinius Syria tligai un itladāgandlu-a.

3. Waigien tl wautliwon-hun kyang kālungdie un ilnagai $\bar{a}$ ung-a ijan.

4. Waigien Joseph ishin Nazareth ilnagai Galilee tligai gu is sta, David gia ilnagai Bethlehem hin kyas Judæa tligai gu is a kulthaian, Darid gia haadgai swon il ijan altha ;

5. Kyang isgien, Mary, nung il înā-ung-kashagan kyē ishin il kālung-die una; waudlu Mary dulthgiāgan.

6. Waigien hawun gu il isūgundān, il kaiungkasas waudlu ēlan.

7. Waigien il ketlagang-an lang-a ilthingāgan; waigien la odō il tiithgalan gien, geudan gin ai sta lthdanūs ai la il dlisdlaian, lthdanou nuhgai à gum lūm la un kang-gang-āwon altha.

8. Waigien althji tligai gu lumadō kechitlē- 
alung kwulthkotis à nāaug-an gien, algwau lumadōgai à ung-a tl lthotsaian.

9. Waigien Shalana gia angelgai swon lia kwulth giāāwon gien, Shalana un yāgudunggai la odō kokēshgadāion; waigien il lthwaugulth-euon-âwon.

10. Waigien nung angelgas hin il shudāwon, Gum lth lthwaugang-ū; gwokeng, gialthang: lā, alth tl gudung-ai lā-euon-ai un, dalung ga lth gialthindang, waigien tl haada wautliwon un isishang:

11. Aiat David gia ilnagai gu Nung alth tl koguns, Christ Shalana isis, dalung un tl kais altha.

12. Waigien adshi ī dalung un Kang-ūdsadāguung; Nung ākzutsūus odō gin tilthgalas gien, geudan gin ai sta lthdanīs à il tîdas dalung keäashang, hin anung angelgas il shudāwon.

13. Waigien umlthistan nung angelgas alth sha-tligai haade shkīlilth-euon-an gien, Shanung-itlagadas à tl kil-lāgan gien, hin tl shoun,

14. Shanung-itlagadas sha āgwi is un lth yāgudung-ū, waigien hēt-tligai gu tl haada alth il gudung-ai las shū lth gudung-ai dlaia isdū, hin tl shouon.

15. Waigien tlistleuon ga angelgasgai shi ga la sta isāwon silīd, lumadō kēchitlē-alung hin gu shudaian, Bethlehem ā wēt talung istīidsun, Shalana tliku gin edan un itil ünshi- 
dultbdas talung kang-gai una, hin gu il shudāwon.

16. Waigien hawīdan il istla-âwon gien, Mary isgien, Joseph ishin, nung āknutsūs geudan gin ai sta lthdanūs à tīdas ishin il kengāwon.

17. Waigien tlistleuon il keng-āwondlu, anung āk kutsūs edē gin la ga tl shudāwon unsta il shu-āwon.

18. Waigien lumadō kēchitlë-alung gin shudai-an ga gudang-an-gai wautliwon wau un kuldang-an.

19. Waigien Mary a gin tl shudaian wautliwon duman kang-an gien, odō il kwulthalan.

20. Waigien lumadō këchitlē-alung silthgang stēlan gien, gin il gudung-âwon isgien, gin il keng-âwon wautliwon ishin sing Shanung-itlagadas un il yagudung-äwon gien, li $\bar{a}$ il kil-lā-âwon, tliku la ga tl shu-āwon gingan-a.

21. Waigien la tl circumcise-die un shin stan-shang-ā-ūlthaiandlu, Jesus hin la t̂l kyādaian, la tl dulthgigai konast nung angelgas il kyādaian ging-an-a.

22. Waigien tliku Moses gia king-go-gungai shouon ging-an, tlistleuon ung tl tla-shkwūnagulai shantlanai waudlu èlandlu, Jerusalem à la tl dläalthaian, Shalana ga la tl isdie una,

23. (Tl ilthansida wautliwon tl kedlaguns $\overline{\mathrm{u}}$ Shalana un nung las hin tl kyādāashang, tliku 
Shalana gia king-go-gung-ai kālung-as gingan-a ;)

24. Waigien kaltsada stung isgien pigeon stung ishin ga hun tl hāasie un $\bar{u}$ tl ijan, tliku Shalana gia king-go-gung-ai shūs ging-an-a.

25. Waigien nung îlthing-a Jerusalem gu ijan, Simeon hin ū il kyāgan; waigien anung ilthing-as lāgan gien, Shanung-itlagadas un il gudung-ai lāgan, waigien Israel haade gudungai wau shagadang-ases da il khatsūgang-an; waigien nung Hants Las la ing-gu ijan.

26. Waigien Shalana gia Christgai il kanggai konast gum il kotulthang-ases Hants Las un il unshidulthdaian.

27. Waigien Hants Las alth temple-gai ai il kudsaian ; waigien tlistleuon Jesus yăalung, tliku lau-gai shūs ging-ån la un il wauawē un, ai la il dlistl-dsāwondlu,

28. Itan la il dligigan gien, Shanung-itlagadas à il kil-lāgan, waigien hin il shouon,

29. Shalana, dung shouon ging-an, wèt gitsadang gudung-ai dlaia alth dung kil-kailung;

30. Hung-ung alth dung gia kogunai lth keng-gun altha,

31. Tl haada wautliwon hang-gu gin dung tla-êlthdaian-a ;

32. Alth tliga kulat haade otgēlths gien, Israel dung gia haadgai is alth un yagudunggai una, hin il shouon. 
33. Waigien la edè gin tl shudaian un il aung ifgien il ou ishin kuldung-ãgan ;

34. Waigien Simeon gūshou lā la à shudāwon gien, hin il ou Mary il shudaian, Gwokeng, Israel haade kwon dl-dulriuns gien tl kalthougadung-ai un ishin anung ākutsūs ijung; waigien hanltha tl gūishue un kang-ūdsada ishin il ijung;

35. Waigien shich dung althandai ai tl kïtsāashang; tliku tl kwon kwulthilths kanggēle una, hin la il shudaian.

36. Waigien Asher guaigang-ai swon, Phanuel gūjang-a jada prophetga ijan, Anna hin kyāgan (il kē-îlthdsilthịgan, waigien il lāinēlan sta tuda jikwau tlal-ung kwulth il ijan,

37. Waigien tuda tläalē stanshang-ā waugu stunshung il dlī-ang-ąn), waigien algasdlu isgien shantlansdlu ishin gium temple.gai sta il kaidang-gang-an, waigien kēsal alth isgien, gin ginang alth ishin singlth-gang il güshugang-an.

38. Waigien althsgai ashkoshgadai gut il katlalthaian gien, Shanung-itlagadas à il killāgan, waigien Jerusalem tl kogundie da tl khatsūgang-an wautliwon à la edē il gūshouon. 39. Waigien tlistleuon tliku Shalana gia kingng-go-gung-ai shūs ging-an il wau-gī-āwondlu, Galilee tligai gwī il stīlthāwon gien, gyanğ ilnagai, Nazareth hin kyāgan, un il istla-āwon. 40. Waigien nung ākeutsūs innastlas gien, il 
dugwiēlan gien, kudang alth il stahgan; waigien Shanung-itlagadas gudung-ai ligai la inggu ijan.

41. Waigien tuda wautliwon gut-hun il yãalung passover lagunung-ai ak Jerusálem à ijang-an.

42. Waigien la da tuda tläalth waugu stangandlu, tl lagunung-kāguns ging-an il istalIthäwon;

43. Waigien tlistleuon shantlanai tlan-ëlandlu, silthgang il stīthāwondlu, Jesus nung ilthing-a kutsū isis Jerusalem gu ung tutlistldaian; waigien il yäalung gum un ūnshidangan ;

44. Waigien tla alth-hun il ising il gudungāwon althū, shin shoshgu il istaläwon; waigien tou-alung shū-wìt isgien, gi un il ūnshitwos shū-wìt ishin, la da il dī-ing-āwon;

45. Waigien tlistlenon la da il dī-inchgāwondlu, Jerusalem gwī silthgang il stîlthāwon gien, la da il dī-ing-āwon.

46. Waigien shin lthōnulth-ēlandlu, templegai à la il kēi-āwon, gin shkotadālē-alung shū il kou-os gien, tl kil à il geulans gien, tla alth il kiānung-gang-an ;

47. Waigien il kil ga güdang-an wautliwon gin un il unshits isgien, tliku hanglthang il shūs ishin un kuldang-an.

48. Waigien tlistleuon la il keng-āwondlu, la un ill kuldung-äwon; Lthken, gushintloụ 
tliku itil un dung ëdung? gwokeng, dung aung isgien lthā ishin gudan stĩ alth dung da di-ing-gun, waigien hin il ou il shudaian.

49. Gushintlou di da dalung dī-ing-ūjang? gum gu Aung-ung gia nai $\bar{a}$ lth itsgai las un dalung unnshidang-üja? waigien hin la il shudāwon.

50. Waigien gin il shudas gum un lang-a il ūnshidang-āwon.

51. Waigien la alth il kaidalthāwon gien, Nazareth gu il istla-âwon; waigien la kilth ging-an il etūgang-an; waigien il ou a gin il shudagang-an wautliwon gudung-ung alth un ung ünshitgang-an.

52. Waigien Jesus kudung-ēlan gien, il dlakonēlan, waigien Shanung-itlagadas isgien tl haada ishin la alth gudung-ai lāgan.

\section{Chapter 3.}

WAIGIEN Tiberius Cesar itlagadēlan sta tuda tlāalth waugu tlēlthaiandlu, Pontius Pilate ishin Judæa un governor-gagandlu, Herod ishin Galilee un tetrarch-gagandlu, waigien Philip il dōn ishin Ituræa isgien Trachonitis un ishin tetrarch-gagandlu, Lysanias ishin Abilene un tetrarch-gagandlu,

2. Annas isgien Caiaphas ishin liplēt un itlagadagandlu, tligunltha John, Zacharias git ijan, à Shanung-itlagadas kil ijan. 
3. Waigien Jordan untlai odō tliga wautliwon is un il katlagan gien, tl dāung-as da kēshgadēlē un gudung-ung stīlthdie baptismgai alth tla geu gu il gushugang-an;

4. Tliku Isaiah nung prophetgas gia kogīnē $\bar{a}$ kil kālung-as ging-an-a ; Shalana gia keu-e lth êlthgìdū, waigien lth lang-a tlayägulthī, hin nung kil swonshung tligunltha gudungägung.

5. Tladanai wautliwon tl stahūdäashang, waigien tldou wautliwon isgien, tliga kēāwos wautliwon ishin, lētga tl isdäashang; ken kitshkājūlung-as ishin yāgulthshang, waigien keu dāung-as ishin ligulthshang;

6. Waigien ga hēnung-asgai wautliwon Shanung-itlagadas gia kogunai keng-ashang, hin kālung-ägung.

7. Althji alth tla il baptize-die un tl shkūleuon la $\bar{a}$ istīidan hin il shudaian, Sik gitalung dalung isis, gīshdu kalthi-yilthdang isang-kasas sta dalung istiid-halang ?

8. Althji alth gudung-ung stilthda un ān las lth inastldū, waigien, Abraham $\bar{u}$ itil aung ijung, gum lth hin gudung-ung-ī ; Shanungitlagadas ashgai kwau-e sta Abraham un gitalung tla-kalthoulthing-ā-gung, hin dalıng lth shudas altha.

9. Waigien wët-hun $\bar{u}$ keutljou-e gin lthkai shkūshang-wē gu tlōdang; althji alth gin Ithkai wautliwon gut gum ān lā innastlans tl 
shkikundals gien, tsanūsi tl sisagung:gung, hin tla il shudaian.

10. Gūsh tlou talung isdie lagung? waigien hin ga shkīlasgai la alth kianang-an.

11. Kodats chi-stung nung dāasdlu, gum nung ga dāans ga il isdie lāgung; waigien tou nung dāas ishin ging-an il ūgai lāgung, waigien hin hanglthang tla il shudaian.

12. Waigien giashouganung-lē-alung ishin tla il baptize-die un istlagan, Nung itlagadas, gūshū talung isdie lāgung? waigien hin la tl shudaian.

13. 'Tliku king-gogung-ai shis telg lth gum tl giashoudang-ī, waigien hin tla il shudaian.

14. Waigien soldier haade ishin la alth kiānang-an, Waigien talung ishin, gushin talung etgai $\bar{u}$ laggung? hin tl shouon. Gum lth tl haada dāa-gīdang-ung-ū, gum lth ging-gangan gin isdang- $\bar{u}$, waigien lth giashougai ung-a gwīlāū, waigien hin tla il shudaian.

15. Waigien tl haada nung da khatsãwon gien, tl wautliwon John Christ itsgai odō kwuIthilgundāu,

16. John hanglthang hin tl wãutliwon shudaian, Lthā ù undl alth dalung baptize-dung; di telg nung dugwias tlou katlang, waigien stashgagai ai sta lang-a tlāadai un n̄ gum di lā-ung-gung; lā $\bar{u}$ Hants Las alth isgien das alth ishin dalung baptize-dāashang ;

17. Waigien il stlai à yhitāwē lang-a ijung, 
yenkien wheat-nai stling-kou-e ung-a il tlashkwuvagulths gien, wheat-nagai ai ishin wheat-gai gudā ung-a il isdie una; wheat-gai kul tlou tsanu gum tl kial-lthingans alth il oggulthdāashang, hin tla il shudaian.

18. Waigien gushou äda $\mathrm{kwon}$ alth isgien, gialthang lā alth ishin 11 haada geu gin il gushou-on ;

19. Waigien Herod nung tetrarch-gas Herodias kwai-ung jã sta isgien, gin däung-a wautliwon il isdaian sta ishin John il stidaiandlu,

20. Herod housen wautelg gin dāung-a isdaian gien, John këchitnai ai il isdaian.

21. Waigien tlistleuon tl haada wautliwon baptize-diēlandlu, Jesus ishin baptize-diēlan gien singlthgang il gushouondln, sha tligai helthalan,

22. Waigien nung Hants Las, kaldsida ging-an ets, la ing-gwī ijan, waigien kil ishin sha sta ijan, Di Git kwōyas ì dung ijung; dung alth di gudung-ai lägung, waigien hin kil shouon.

23. Waigien Jesus, tlistleuon gin tla il shkota-dìdandlu, la da tuda tlāale lthonālan, waigien Joseph git il ising tl gudung-gangan ;

24. Waigien Joseph Heli git ijan, Heli ishin Matthat git ijan, Matthat ishin Levi git ijan, Levi ishin Melchi git ijan, Melchi ishin Jannai git ijan, Jannaị ishin Joseph git ijan, 
25. Joseph ishin Mattathias git ijan, Mattathias ishin Amos git ijan, Amos ishin Nahum git ijan, Nahum ishin Esli git ijan, Esli ishin Naggai git ijan,

26. Naggai ishin Maath git ijan, Maath ishin Mattathias git ijan, Mattathias ishin Semein git ijan, Semein ishin Josech git ijan, Josech ishin Joda git ijan,

27. Joda ishin Joanan git ijan, Joanan ishin Rhesa git ijan, Rhesa ishin Zerubbabel git jjan, Zerubbabel ishin Shealtiel git ijan, Shealtiel ishin Neri git ijan,

28. Neri ishin Melchi git ijan, Melchi ishin Addi git ijan, Adlli ishin Cosam git ijan, Cosam ishin Elmadam git ijan, Elmadam ishin Er git ijan,

29. Er ishin Jesus git ijan, Jesus ishin Eliezer git ijan, Eliezer ishin Jorim git ijan, Jorim ishin Matthat git ijan, Matthat ishin Levi git ijan,

30. Levi ishin Symeon git ijan, Symeon ishin Judas git ijan, Judas ishin Joseph git ijan, Joseph ishin Jonam git ijan, Jonam ishin Eliakim git ijan,

31. Eliakim ishin Melea git ijan, Melea ishin Menna git ijan, Menna ishin Mattatha git ijan, Mattatha ishin Nathan git ijan, Nathan ishin David git ijan,

32. David ishin Jesse git ijan, Jesse ishin Obed git ijan, Obed ishin Boaz git ijan, Boaz 
ishin Salmon git ijan, Salmon ishin Nahshon git ijan,

33. Nahshon ishin Amminadab git ijan, Amminadab ishin Arni git ijan, Arni ishin Hezron git ijan, Hezron ishin Perez git ijan, Perez ishin Juda git ijan,

34. Juda ishin Jacob git ijan, Jacob ishin Isaac git ijan, Isaac ishin Abraham/ git ijan, Abraham ishin Terah git ijan, Terah ishin Nahor git ijan,

35. Nahor ishin Serug git ijan, Serug ishin Reu git ijan, Reu ishin Peleg git ijan, Peleg ishin Eber git ijan, Eber ishin Shelah git ijan,

36. Shelah ishin Cainan git ijan, Cainan ishin Arphaxad git ijan, Arphaxad ishin Shem git ijan, Sbem ishin Noah git ijan, Noah ishin Lamech git ijan,

37. Lamech ishin Methuselah git ijan, Methuselah ishin Enoch git ijan, Enoch ishin Jared git ijan, Jared ishin Mahalaleel git ijan, Mahalaleel ishin Cainan git ijan,

38. Cainan ishin Enos git ijan, Enos ishin Seth git ijan, Seth ishin Adam git ijan, Adam ishin Shanung-itlagadas git ijan.

\section{Chapter 4.}

W AIGIEN Jesus Jordan sta silthgang stēlan gien, Hants Las alth il stā-ūgãgan, waigien shin tläale stunshung gut tligunltha-et nung Hants Las il al-kägan, 
2. Waigien hetgwaulana la edẽ kil-kang-an. Waigien althsgai sing-ai gut gum il lthdanūung-gang-an; waigien tlistleuon tlan ëlandlu, il kwotagalan.

3. Shanung-itlagadas Git dung isdō lth, adshi kwau-ē, Sllibli-gēltha, hin shudi, waigien hin hetgivaulana il shudaian.

4. Gum rung haada shibli alth swonan hēnung-ang- fasbang, hinū kālung-āgung, hinū Jesus hanglthang il shudaian.

5. Waigien shi ga la il al-kulthaian gien, moment swonshung gut kingdomgai wautliwon hêt-tligai gu isis la il kendaian.

6. Waigien hetgwaulana hin il shudaian, Adshi dugwigai wautliwon is isgien, un yāgūdung-gai ishin, dung ga lth isdāashang; di gia tl isdaguni altha: waigien nung ga isdie da di guduns ging-an wàu ga lth isdalthingāgung.

7. Althji alth di hang-gu singlthgang dung gushusdlu, wautliwon dung gia isisang, hin la il shudaian.

8. Shalana Itlagadang ā singlthgang dung gushus gien, la un swonan dung lthong-gwilie lāgung, hinū kālung-āgung, hin Jesus hanglthang il shudaian.

9. Waigien Jerusalem ā la il āl-kaidan gien, templegai tla's ing-gu la il isdaian, waigien hin la il shudaian, Shanung-itlagadas Git dlung isdō lth, sta agung kātãalth ; 
10. Gyang angels-gai duman dung il kengalthshang ;

11. Waigien stlang alth dung tl dligungashang, gum kwau ai dung stai stōwong-ai una, hin kālung-āsi altha, hin la il shudaian.

12. Shalana Itlagàdang edè dung kil-kanggai gum lä-ung-gung, hin tl shugung, waigien hin Jesus hanglthang il shudaian.

13. Waigien tlistleuon hetgwaulana il edè kilkang - gai wautliwon tlan èlthdaiandlu, kwai-umdsiwun la sta il kaidan.

14. Waigien Jesus Hants Las gia dugwigai alth Galilee tligai gwi stēlan; waigien la alth gialthing-e tligai ashkwau-un gùt king-agan.

15. Waigien tlagia synagogne-nagai $\bar{a}$ gin tla il shkotadagang-an gien, tl wautliwon la un yāgudang·an.

16. Waigien Nazareth gu il katlagan, gyagu il inastlaian-a ; waigien tliku il edang-an gingan sabbath shantlanai ijandlu synagogue-nagai ai il kudsaian, waigien kōgīn il līdidie un il giāagan.

17. Waigien Isaiah nung prophetgas gia kōgīnē la ga tl isdaian. Waigien kōgīnē il tla-yāgālan gien, gy'a gu hin kālung-āsi il kēaian,

18. Shalana Hants di ing-gu ijung, gialthang lā alth ga kong-ushgitaigasgai geu gu lth gushue un di kuts il tōdagun altha; ga kechidasgai kil-isāgai un isgien, ga hang-ka- 
gasgai gin keng-ashang ga lth shougai un ishin, ga tligiasgai lth istiidai un ishin,

19. Shalana gia tudie alth gudung-ai ligai unsta lth shougai un ishin, di il kil-kaidun, hin kālung-āgan.

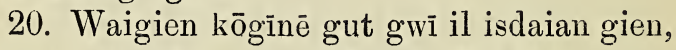
duman nung kens ga silthgang il isdaian gien, il kāwon; waigien synagogue-nagai à ga isgai wautliwon il khatsgadan.

21. Aiat tliku adshi kālung-e shūs ging-an ets dalung gudung-gung, waigien hin tla ga il shu-īdan.

22. Waigien tl wautliwon tliku il shouon guvūlāgan, waigien gushou lā il helthi sta ijan un tl kuldung-āgan; Gum gu unis Joseph git isang-üsh? waigien hin tl shouon.

23. Waigien hin tla il shudaian, Tla nung tl-ligal-leggas, agung 1th tl-ligulth; tliku Capernaum gu gin edan talung gudung-gun ging-an lth adlun tlig-ang alth wau, hin gushou et alth di dalung shudāases un di unshidung, hin tla il shudaian.

24. Waigien hin ishin il shouon, Yenkien dalung ga lth shugung, Gum nung prophetga tligang gu tl gūsānāwaugung-ung-gung.

25. Waigien yenkien $\bar{u}$ hin dalung ga lth shugung, Elijah nūt Israel tligai gu tl dsūlthin-ga kwonan, tuda lthōnulth isgien, khōng kē-tlū-unilth ishin sha-tligai wau tl shkoshgadan gien, tligai ash-kwau-hun gut tou hīlāwondlu-a ; 
26. Waigien gum ashgai siron-ā-hun Elijah tl kaidang-an, nung dsūlthin-ga Zarephath, Sidon tligai gu is, a swonan la tl kil-kaidan.

27. Waigien Elisha nung prophetgas nūt tligai gu leprosy alth tl kwon stigan; waigien gum tl swon-liun shkwunagulthang-an, tlan Naaman, nung Syria haadas, swonan shkwunagalan, waigien hin tla il shudaian.

28. Waigien tl wautliwon synagogue-nagai $\bar{a}$ ijan kalthi-yilthd-euonan, adshi tl gudangandlu-a :

29. Waigien tl tljūgiāgan gien, ilnagai sta kyā ga la tl dang-an gien, gya ing-gu ilnagai althin-daian stulai kwul à la tl kāgan, sta la tl kātāalai una.

30. Waigien tla kalthgut-hun il kāgan gien, il kai-dan.

31. Waigien Capernaum ilnagai, Galilee tligai gu ijan, un il katlatalan. Waigien sabbath shantlanai ai gin tla il shkotadagang-an;

32. Waigien gin tla il shkotadaian un $\mathrm{tl}$ kuldang-an ; dugwia alth il gushouon altha.

33. Waigien nung ilthing-a synagoguenagai $\bar{a}$ jjan, waigien hants gum shkwūnang la à ijan; waigien hin kwunan il shouon,

34. Güshāku itil un dung katlang, Jesus, Nazareth haadas? itil hīlūdie un gu dung katla-üsh? nung dung isis un ū di ünshidung; Shanung-itlagadas gia Nung Las dung ijung, hin kwunan il shouon. 
35. Waigien Jesus il stidaian, Tlan th gushu, waigien la sta lth kāgwulth, waigien hin il shouon. Waigien tlistleuon nung hants däung-as tla shu il tl-dildulthdaiandlu, la sta il kāgwaulan, waigien gum tligwon la il edang-an.

36. Waigien tl kuldung-ā-ōdson-on gien, gudā tl gushouon, Gūsh kīl $\bar{u}$ à jung? dugwìa alth hants gum shkwunans tliku il shudas. ging-an tla sta kāgwulgung-gung, waigien hin tl shouon.

37. Waigien la alth gialthing-e tliga wautliwon wau odō ijan ai king-āgan.

38. Waigien synagogue-nagai sta il kāgwaulan gien, Simon gia nai ai il kudsaian. Waigien Simon jūnan sti kina alth sti-euonan; waigien la sing la à tl gushouon.

39. Waigien la sha il giāagan gien, sti kīnas il stidaian, waigien lang-a gū-ēlan; waigien hawīdan il kalthāwon gien tla il lthdanū-daian.

40. Waigien tlistleuon kokēlthagadalandlu ga tou-i sti gut īlā et alth stis la à tl kelthilang-an; waigien tl wautliwon gu-hun il tltulthdagang-an, waigien tla il tl-ligalan.

41. Waigien tl kwon sta ishin hants dāunga ijan, waigien kwunan tl gushouon gien, Shanung-itlagadas Git $\bar{u}$ dung ijung, hin tl shouon. Waigien tla il stidaian gien, tl gushue $\bar{a}$ il kēgadalan, nung Christgas il is un tl ūnshidan altha. 
42. Waigien tlistleuon sh:ıntlanandlu, il kāgwulthāgan gien, tligunltha à il kaidan; wdigien tl shkūlas la da kang-an gien, la un tl istlagan, waigien stagang il kaidie da gum tl gudung-ang-an.

43. Waigien hin tla il shudaian, Lana kulatit ishin Shanung-itlagadas gia kingdomgai alth gialthing-e las alth tla geu gu lth gushue lāgung; un di tl kil-kaidun altha, hin tla il shudaian.

44. Waigien synagogue-nai Galilee tligai gu ijan à tla geu gu il gushugang-an.

\section{Chapter 5 .}

WAigies la odō tl shkūlēlan gien, Shanung-itlagadas kil tl gudang-andlu, Gennesaret shu-e jin-gu il giāang-an;

2. Waigien kontlu kê-stung shu-e $\bar{a}$ gīhaguns il kang-an; ga ãada-lēgasgai tlou wau sta istāalan gien, āade ung-a tl dlan-an.

3. Waigien kontlū-e ke-swon Simon gia ijan gwe il katligan, waigien kotgwī tligai sta la il tlū-kusalan. Waigien il kouon gien, kontlū-e gwau sta tl shkūlas gin il shkotadaian.

4. Waigien tlistleuon tlan il gushouondlu, Kotga lthdīing-gai ā lth tlū-kusi, waigien chān ga āade ung-a isdi, dung āadie una, hin Simon il shudaian.

5. Nung itlagadas, al shoshgu talung āadaHA. 
gun, waigien gum gin talung kntliidang-gun ; waikianan tliku dung shūs ging-an chān ga àade ung-a lth isdāashang, waigien hin Simon hanglthang shouon.

6. Waigien tlistleuon hin il wau-awondlu, shkolthung kwon-euon il chitlāwon, waigien ãade lang-a adsilthidawon;

7. Waigien kontlū kulat gwau tou-alung isāwon hung-a il tlū-uldung-āwon, la da il tlāadawe una. Waigien il tlū-katla-âwon gien, kontlū-e ke-stung-un il sta-hūdāwon, waigien chahatēidan.

8. Waigien tlistleuon Simon Peter kangandlu, Jesus kwolī hetgu il tīgūstlīi-aian, Shalana, di sta lth kaid, nung ilthing-a dāung-a di isis altha, waigien hin il showon.

9. La isgien, la alth ga ijan wautliwon ishin, shkolthung kwon-enon tl isdaian un kuldungägan altha;

10. Waigien James isgien John ishin, Zebedee gitalung ijan, Simon alth gudī āadlēgagan ishin kuldung-âgan. Gum lth lthwaugang; adshi sta tl hãada dung isdāashang, waigien hin Jesus Simon shudaian.

11. Waigien tlistleuon kontlue dit-gwi ga ung-a il isdāwondlu, gin wautliwon il tlistlāawon gien, la dlā il istiidāwon.

12. Waigien bawun ilnagai swon gu il ijundān, nung îlthing-a leprosy alth stāingăgan gu ijan ; waigien tlistleuon Jesus il kang-andlu, 
il tīgīistlīaian gien, la à singlthgang il gushouon, Shalana, dung gwīlasdlu di dung tlashkwunagal-lthing-āgung, waigien hin il shouon.

13. Waigien stlang il jistlaian gien, la inggu il tl-dulthdaian, Di givūlāgung; shkwunagul-ltha, waigien hin il shouon. Waigien hawīdan leprosy-gai la sta gūēlan.

14. Waigien gum nung ga il shudalthangan, Kaid-ltha, waigien lth nung liplētgas agung kendi, waigien tliku Moses tuking-go-gang-an ging-an lth dung shkwunagulths alth gin isda, dung ligulths un tl ūnshidai una, waigien hin il shouon.

15. Waigien wautelg āgwī la alth gialthing-e king-ā-kokūstlu-aian ; waigien tl shkūl-euon il kil gudung-ai un isgien, tla il tl-ligulai un ishin, gudā ijan.

16. Waigien tla sta tligunltha à il kaidan gien, singlthgang il gushonon.

17. Waigien althsgai shantlanai swon gut gin tla il shkotadaian; waigien Pharisee haade isgien, king-goguns shkotadālē-alung ishin gu tiō-daian, waigien Galilee gu isgien, Judæa gu ishin, Jerusalem gu ishin, lana ijan wautliwon sta u tl ijan; waigien tla il tl-ligulai un Shalana gia dugwigai la alth ijan.

18. Waigien tl ilthansida sti dliida alth nung stis ūnchāang-ū ing-gu isis la à tl isdaian; waigien anā ga la tl isdas gien, la hetgu il āügai da tl gudang-an. 
19. Waigien tl shkūlas alth gum tliku anā ga la tl isdalthing-e kang-gang-andlu, nas inggwì ga tl isdlalthaian gien, Jesus tla yākosĩa ijan hetgu nas ing-gu sta ūnchāangwē dungalth la tl yhitadsaian.

20. Waigien tl yetdas il kang-andlu, Nung îlthing-as, dung dāung-as gagakung-gung, hin il shouon.

21. Waigien tukālung-lēalung isgien, Pharisee haade ishin, ede $\bar{a}$ kāūgìidan, Gishd $\bar{a}$ is $\bar{u}$ gushou dāung-a shudang? Gishdū tl dāung-as gakang-lthing-āgung, tlan Shanung-itlagadas swonan-a? waigien hin tl shouon.

22. Waigien gin à tl kāūgas un Jesus unshidalandlu, hin hanglthang tla il shudaian, Gūsh odō dalung kwūlthilgung ?

23. Dung dāung-as ga gakung-gung, hin shougai isgien, Kālthūltha waigien lth $k \bar{a}$, hin shougai ishin, gīshgai-ū yang-alang?

24. Waigien nung haada Git hët-tligai gu tl dāung-as gakang-gai un dugwias un dalung unshidai un lth shugung, hin tla il shudai-an; Kālthūltha, waigien lth ünchäang-we ung-a isdi, waigien lth nai $\bar{a}$ ung-a kaid, hin dung lth shudang, (waigien hin nung sti dlïidas il shudaian).

25. Waigien hawīdan tla hang-gu il kālthāwon gien, gin ing-gu il tīdaian ung-a il isdaian gien, nai à ung-a il kaidan, waigien Shanungitlagadas un il yāgūdang-an. 
26. Waigien tl wautliwon kuldung-āgan, waigien Shanung-itlagadas un tl yāgūdang-an ; waigien tl lthwaugulth-euon-an, Aiat gin akaia talung keng-gun, waigien hin tl shouon.

27. Waigien adshi dlā il kagwaulan, waigien gya gu tl giashous gu nung giashouganung-lēga kou-os il kang-an, Levi hinū il kyāgan; Di dlā lth kā, waigien hin la il shudaian.

28. Waigien gin il tustl-ōdsou-on gien, il giāagan, waigien la dlā il kaidan.

29. Waigien Levi gyang nai ä lagunung-euon la un tloulthaian; waigien ga giashouga-nunglēga shkūl-euon isgien, tl kulat ishin, lthdanūe un tla alth tlō-daian.

30. Waigien Pharisee haade isgien, tlagia tukālung-lēalung ishin lagia disciplesgai un gudung-ai stigan, Gushintlou giashouganunglê-alung isgien, ga dāung-asgai ishin alth dalung lthdanūgung? waigien hin tl shouon.

31. Waigien Jesus hin hanglthang tl shudaian, Ga althinsgai $\bar{u}$ gum tla nung tl-ligallēgas ga stalthagung-ung-gung; ga stisgai tlou ga stalthagung-gung.

32. Gum ga lasgai un ai-ing-ai un lth katla-ang-gung ; ga dāung-asgai gudung-ung stilthdie un $\bar{u}$ lth katlang, hin tla il shudaian.

33. Waigien hin la tl shudaian, John gia disciplesgai kesalgìgung-gung, waigien singlthgang tl gushugigung-gung; Pharisee haade gia disciplesgai ishin ging-an êdung-gung; 
dung gia disciplesgai tlou Ithdanñgung-gung, hin la tl shudaian.

34. Waigien hin Jesus tl shudaian, Hawun nung jatanēlths tou-alung wau kwulth iskialthk gu tla dalung kēsal-ralthing-ā-üsh ?

35. Waigien nung jatanêlths tla sta tl isdāases shantlanai isisang, gai sing-ai gut tl kēsalashang, hin Jesus tl shudaian.

36. Waigien kēgang alth tla à il gushouon; Gum nung swonshung-hun kōdats outla sta gin dung-gitlas gien, kodats kēga à il gūshgīdung-ung-gung; ging-an il wausdlu, nung outlas il dung-ilth-gwai-ishang, waigien nung outlas sta ga isgai ishin gum nung kēgas un lä-ungashang.

37. Waigien gum nung swonshung-hun wine outla gin kul kēga ai isdagung-ung-gung; ging-an il wausdlu, wine outlas gin kul is tla-kwong-ashang, waigien $\overline{\mathrm{u}}$ ging-gūashang; waigien gin kul is ishin güashang.

38. Waigien wine outla gin kul outla ai tl isdie $\bar{u}$ lāgung.

39. Waigien gum nung swonshung-hun wine kēga nīlthsdlu, ga outla ga stalthagungung-gung; ga kēgas lang il shūs altha, hin il shouon. 


\section{Chapter 6.}

WAIGiEN sabbath shantlanai ai com kwulthkotajai kalthgut il kāgan; waigien lagia disciplesgai corn-gai dung-gïidan gien, gutgwī il tla-hanang-wos gien, il tã-āwon.

2. Gushintlou tuking-go-gung-ai shus ilā sabbath shantlanai ai dalung waugung? waigien Pharisee haade ilthi hin shouon.

3. Waigien Jesus hin hanglthang tl shudaian, Tlistleuon David kwōtagālandlu, tliku il waugan gien, tliku la alth ga ijan-gai ishin waugan, gum gu dalung lididang-üja?

4. Shanung-itlagadas gia nai ai il kudsaian, waigien shibli las il isdas gien, il tāgan, waigien la alth ga ijan-gai ga ishin il isdaian; althji tlan ga liplètgas swonan tugai lang tuking-go-gung-ai shouon, hin Jesus shouon.

5. Nung haada Git $\bar{u}$ sabbath shantlanai un itlagadāgung, hin ishin tla il shudaian.

6. Waigien housen sabbath-gagandlu, synagogue-nagai ai il kudsaian gien, gin tla il shkotadaian; waigien nung îlthing-a sōolgūust stlai yhilgala à ijan.

7. Waigien tukālung-lêalung isgien, Pharisee haade ishin, la à lthotsaian, sabbath shantlanai ai la il tl-ligulai tl kang-gai una; tliku la hanltha gushoulthing-e tl kēie una.

8. Waigien tliku tl guduns un il ūnshidan; 
Giāaltha, waigien lth tla yakosīa giāia, waigien hin nung stlai yhilgalas il shudaian.

9. Waigien il giāagan gien, tla yakosia il giāagan. Waigien hin Jesus tl shudaian, Sabbath shantlanai ai gin lā isdie gu lā ? gin dāung-a isdie gu lā? nung hēnung-gai kngundie gu lā? nung tỉie gu lā? dalung alth lth kiānung-gung, hin tla il shudaian.

10. Waigien $t l$ wautliwon gut il kendagwongan gien, Stlang lth jistla, hin la il shudaian. Waigien ging-an il waugan, waigien il stlai langa ligalan.

11. Waigien la un tl kalthi-yilthd-euonan, waigien tliku Jesus tl edalthing-ai à gūtga tl kāūgaian.

12. Waigien althsgai shantlanai gut singlthgang gushue un tldāwe à il kaidan; waigien al shoshgu Shanung-itlagadas a singlthgang il gushouon.

13. Waigien shantlandlu, gyang disciplesgai un il lthai-aian; waigien tla sta tl tlāalth waugu stung il kenstaian, waigien apostles hin la il kyādāwon;

14. Simon Peter hin ishin la il kyadaian isgien, Andrew il don ishin, James ishin, John ishin, Philip ishin, Bartholomew ishin,

15. Mat thew ishin, James Alphæus git ijan ishin, Simon Zealot hin kyāgan ishin,

16. Judas James git ijan ishin, Judas Iscariot la alth nung giadahgan ishin; 
17. Waigien la alth il kāalțìawon gien, tliga yā gu il giāang-an, waigien lagia disciplesgai shkūl-euon-an, waigien Judæa tligai ashkwauhun sta isgien, Jerusalem sta ishin, Tyre isgien Sidon ishin ai-awe gust ijan sta ishin, tl shküleuon il kil gudung-ai un isgien, tl stis tlang-a il tla-ngīstlie un ishin istlagan ;

18. Waigien hants gum shkwunans alth ga gudung-ai lthkwīdas ishin ligalan.

19. Waigien tl shkūlas wautliwon il tl-tlie da gudang-an; dugwia la sta ijan gien, tl tlangīstla-ōdsou-on altha.

20. Waigien gyang disciplesgai il khatsgīlthdaian gien, hin il shouon, Dalung kongushgitaigas ū lāgung; Shanung-itlagadas gia kingdomgai dalung gia isis altha.

21. Wèt dalung kwots ī lāgung, dalung skisūases altha. Wēt dalung saigds ū lāgung, dalung kah-ases altha.

22. Nung haada Git agunan tl haada dalung telg kens gien, stagang dalung tl dans gien, ishin dalung tl kil-ēdans gien, gin dāung-a tl dang-guns ging-an dalung kyē ishin tl dansdlu, dalung ù. lāgung.

23. Gai sing-ai gut lth tlika gwūlā-ū ang-a kai-ang-ū; sha-tligai gu dalung gia giashougai euonsi altha, wai ging-ān tl aung-alung ishin ga prophetgasgai etdagang-an altha.

24. Dalung itlagadas un tlou gin ga lthwaugīgāashang! dalung gudung-ai wau shatusgīs altha. 
25. Wèt dalung skisawos un gin ga lthwaugīgäashang! dalung kwotagulthses altha. Wèt dalung khas un gin ga lthwaugịan-ashang! dalung kogun-inas gien ishin dalung saigãases altha.

26. Dalung alth tl haada wautlwon gialthindie lasdlu, dalung un gin ga lthwaugiganashang! ging-an tl aung-alung ga prophetga kilthgadung-as un etdang-an altha.

27. Waikian di kil dalung guduns hin Ith shudang, Dalung ga hānāgādasgai lth kwoyādì, dalung telg ga kensgai un lth là-un,

28. Dalung ga lānūsgai à lth gushou là shīdù, dalung ga dāagudans sing lth gushūū.

29. Dung kunsut nung shkwudasdlu, inawē ishin lth la gwi èlthda; waigien kōdats chikōnas dung sta nung isdasdlu, akwon lth kōdadsai ishin ung-a il isdada.

30. Dung alth gin ga tl kiānuns wautliwon ga lth gin isda ; waigien gin dung dāas dung sta nung isdasdlu lth, gum silthgang ginung-ung.

31. Waigien tliku dalung un tl etgai da dalung guduns ging-an lth tla un etū.

32. Dalung ga kwōyādasgai swonan dalung kwoyādasdlu, ging-gang-an $\bar{u}$ èdung, ga dăung-asgai ishin tl kwoyādasdlu, gai ishin tl kwoyädagung-us altha.

33. Dalung un ga lasgai swonan un dalung. lasdlu, ging-gang-an $\bar{u}$ ēdung, ga dāung-asgai ishin ging-ān èdung-us altha. 
34. Waigien dalung ga ga giashou-ashang dalung guduns swonan dalung gia-shildadasdlu, ging-gang-an $\bar{u}$ èdung; ga dāungasgai ishin ga dāung-asgai gia-shildadagunggung, housen silthgang tl da-êlē una.

35. Dalung ga hānāgādasgai lth kwoyãdı ang-a tla un lā-ù, ishin lth tl gia-shildadū, gum lth kai-inslang-ī; waigien dalung gia gia-shougai euon-shang, waigien sha āg wi Nung ets gitalung $\bar{u}$ dalung isisang; gum ga kil-lansgai isgien, ga dāung-asgai ishin un il kung-gudung-as altha.

36. Tla kalthshint th gudung-ī, dalung Aung tla kalthshint gudung-guns ging-ān-a.

37. Waigien lth gum tu-ging-kilisalung.. ung- $\bar{u}$, waigien dalung ishin gum tl gingkilisalung-ung-ashang; gum lth tl tlü-unshkotalthang- $\bar{u}$, waigien gum dalung ishin tl tlūunshkotalthang-ashang; tla kalthshint lth gudung-u, waigien dalung kalthshint ishin $t$ gudung-ashang;

38. Tlla ga lth gin isdu, waigien dalung ga ishin gin $\mathrm{tl}$ isdāashang; gin kwīdie las isgien, ing-gu tl-duldung-as ishin, giidias ishin, wau sta knu-ūdas ishin, dalung shkeu ai tl isdāashang. Tliku tla un gin dalung kwidas ging-an dalung un ishin tl kwìdāases altha, hin Jesus shouon.

39. Waigien kēgang alth ishin tla āil gushouon, Ga hang-kagasgai gu ga hang-kaga 
al-istal-lthing-a ? gum gu tl stung-hun gin hēla ai hāwīdsang-ashā-ūsh ?

40. Nung disciple-ga gum itlagidang telg èdung-ung-gung; nung un gin shkot-ilthdasdlu tlou, ittlagidang ging-an etishang.

41. Gushintlıu dung kwai hung-i ā shkelgìishguns dung khatsgidung, waigien dung hungi a tlimit isis tlou gum un dung ūnshidanggung?

42. Di kwai is, dung hung-i à shkel-gīishguns wau sta dang-a di isdada, gushintlou hin kwai-ung dung shudalthing-āgung, waigien dung hung-i à tlimīt isis gum dung kengung-gung? Dung gudung-ai stung-gung! hung-ung sta lth tlimadai dang-tlagang, waigien itan dung kwai hung-i à shkelgi-ishguns tlāalgwĩ dang-gai un dung hung-ā kang-ūlāgulthshang.

43. Gum gin lthkai lā gut ān dāung-a innastlagnng-unsi altha; gin lthkai dāung-a gut ishin gum ān lā inastlagung-ung-gung.

44. Gin lthkai wantliwon ānē alth un tl unshidung-us altha. Gum gin lthkai ki sta tl hāada fig isdagung-ung-gung, gum gin lthkai ki sta ishin grape tl isdagung-ung-gung.

45. Nung hāada lā il gudung-ai ā gin kwōyã lā isis sta gin lā isdagung-gung; nung hāada dāung-a ishin gin kwōyā dāung-a sta gin dāung-a isdagung-gung; gin alth il gudung-ai stā-ū-gas sta gin unsta il shugung-us altha. 
46. Shalana, Shalana, gushintlou hin di daling kyädas gien waigien gum tliku lth shūs ging-an dalung waugung-ung-gung ?

47. Nung-hun di un katlas gien, di kil il guduns gien, ishin ging-an il wausdlu, tliku il ets dalung lth kendãashang;

48. Nung ilthing-a nuh tloulthas gien, chān ga il lthaidsas gien, tīsh ing-gu kwau-wong-ai il kwūnlthin-gadaian ging-ān-ū il ēdung ; waigien tlistleuon untlai kãgan gien, nai ai kwahodāwon-dlu, gum ù ging-yilthdalthing-ang-an; tloulthie lāgani altha.

49. Waigien di kil nung guduns gien, gum ging-an il wau-unsdlu, nung ilthing-a kwauwong-ai ädan tligai ing-gu nuh tloulthaian ging-an $\bar{u}$ il ēdung; waigien ai kwau-odāwon dlụ, hawīdan hündsaian, waigien nai yenkien gàwon, hin Jesus shouon.

\section{Chapter 7.}

TL haada geu gu il gushu-elthdaiandlu, 1 Capernaum ai il kudsaian.

2. Waigien nung centurion-ga gitsadang kwōyādaian stigan gien kotulthdalan.

3. Waigien tlistleuon Jesus alth gialthing-e il gudang-andlu, Jews haada k'èas la à il kilistiidan, waigien il gîtsada il kogundie da la alth il kiānang-an.

4. Waigien tlistleuon Jesus un il istla-āwon- 
dlu, kwunan la alth il kiānung āwon, Adshi la un dung isdie un il lāgung ;

5. Itil gia gwai-gang-ai il kwôyādas gien, la kodan ishin itil gia synagogue-nagai tloulthagun altha, waigien hin il shu-äwon.

6. Waigien Jesus la alth kaidāwon. Waigien tlistleuon nai un il dōng-êlandlu, nung centurion-gas tou-alung la à kilistïidan, waigien hin la il shudāwon, Shalana, gum lth gudung-ung lthkwidadang; di kwulth dung kudsie un gum di lāans altha;

7. Althji althū ltha kodan dung a a kogai un gum di lâ-ung-ung lth gudung-gun; hing-an Ith gushu, waigien di gitsada ngistlāashang.

8. Di un ishin tl itlagadas altha, waigien di ishin soldier haade un itlagadāgung; waigien, Kaidltha, hin nung swon lth shudasdlu, il kaidung-gung; Halüt, hin ishin nung swon lth shudasdlu, il katlagung-gung; Adshi lth isdi, hin ishin gitsadang lth shudasdlu, il isclagung-gung, hin il shu-āwon.

9. Waigien tlistleuon Jesus adshi gudangandlu, la un il kuldung-ägan, waigien ga shkūla la dla istals gwī ung-a il dlisdlaian, Israel haade shu-hun gum hin nung yetdatlju lth keng-ung-gun dalung ga lth shudang, waigien hin tla il shudaian.

10. Waigien ga il kilistīidan-gai nai $g w \bar{i}$ silthgang stēlan, waigien nung gîtsadas ligulths il keng-âwon. 
11. Waigien gum jīng-ang-gundān Nain hin lana kyā à il kaidan; waigien lagia disciplesgai isgien tl shkūl-euon ishin la alth ijan.

12. Waigien ilnagai keu-e un il dongēlandlu, nung kotalā tl ādlāwaugan, waigien tlan la swonan il ou kaian gien, il on dliangan ; waigien ilnagai haade kwon la alth ijan.

13. Waigien Shalana il kang-andlu, la kalthshint il gudang-an, Gum lth sailthang, waigien hin la il shudaian.

14. Waigien äanan il katlagan gien, gin alth la tl ahgundals ing-gu il tl-dulthdaian, waigien ga ahgundalan-gai tljügiāagan. Nung itaninās, kalthultha, hin dung lth shudang, waigien hin il shouon.

15. Waigien nung kotulāgan yā kouton gien il gushu-ïan. Waigien il ou ga la il isdaian.

16. Waigien tl wautliwon lthwaugalan; waigien Shanung-itlagadas un tl yăgudang-an, Nung prophetga euon ù itil shu isilgung, hin tl shouon gien, Shanung-itlagadas gyang haadgai keng-atlang, hin ishin tl shouon.

17. Waigien adshi alth gialthing-e Judæa tligai ashkwau-hun gut isgien, odō tliga ijan wautliwon èt ishin lang-a king-ā-kokūstlūaian.

18. Waigien John gia disciplesgai a gin is wautliwon la ga shudaian.

19. Waigien John gyang disciples-gai stung un aiang-an gien, Shalana à la il kilistiidāwon, 
Nung katla-ung-kasas g" dung is? nung kulat ga gu talung khatsū-ash ? waigien hin il shu$\bar{a}$ won.

20. Waigien tlistleuon tl ilthansidas la un istlagandlı, hin il shū-äwon, John Baptist dung $\bar{a}$ itil kilistīidun, Nung katla-ıng-kasas gu dung is? nung kulat ga gu talung khatsūash? hin il shugung, hin il shūāwon.

21. Althsogai ashkoshgnt güt tl sti kwon i-gien, tl stigaga ishin, hants dāung-a gi à isisgai ishin, il tl-ligalan; waigien ga hangkaga kwon ishin hung-i il tla-helthilthgaian.

22. Waigien hin hang-lthang la il shudāwon, Istiidultha, waigien gin dalung kens isgien, gin dalung guduns ishin John ga lth shūdū ; ga hang. kagasgai $\bar{u}$ hung-i helthilthgang, ga kwolū kōdas istalgung-gung, leprosy alth ga stisgai shkwūnagulgung, ga geudanasgai gin gudung-gung, ga kotulthgiasgai kālthougadung-gung, waigien ga kong-ushgitaigasgai geu gu gialthang lä alth $t l$ gushug'mg.

23. Waigien gum di nung gū-dāung-uns $\bar{u}$ lā-gung, hin hanglthang la il shudāwon.

24. Waigien tlistleuon John la à ga kilis.. tīidan-gai istīidandlu, ga shkūlasgai ga John alth il gialthindiidan, Güsh dalung kang-gai un $\bar{u}$ tligunltha $\bar{a}$ dalung istīidang? kuncbich tudsou alth hu-yhilduns gwau?

25. Gūsh kang-gai un tlou dalung ijang? nung ilthing-a gin lthtuna giandas gwau? 
Gwokeng, gin lā ga giandasgai isgien, tou lā ga tasgai ishin, ga king-gas gia nai à ijung.

26. Güsh kang-gai un tlou dalung ijang? nung prophetga gwau? Ang, nung prophetga telg āgwi nung et dalung keng-āgan dalung: ga lth shudang.

27. Althunis edē $\bar{u}$ hin kalung-ägung, Gwokeng, dung konast gitsadang lth kilkaidung, waigien dung gia kell-e dung konast il elthgīdā-ashang, hin kālung-āgung.

28. Tl jāada ga kaiwosgai shū gum John telg nung edang-gung; waikian Shanungîtlagadas gia kingdomgai gu nung îkutsū isis hawun la telg edung dalung ga lth shīgung, hin Jesus shouon.

29. Waigien tl haada wautliwon isgien, giashouganung-lēalung ishin, tlistleuon tl gudangandlu, Shanung-itlagadas tl yetdaian, John gia baptism-gai alth tl baptize-diāgan altha.

30. Waigien Pharisee haade isgien, tukinggo-gang-lēalung ishin, Shanung-itlagadas kil ga gwou-aian, waigien gum tla il baptizedang-an.

31. Waigien Jesus hin $t l$ shudaian, Gūsh ging-an $\bar{u}$ wēt hāade lth etdāashang, waigien güsh ging-an $\bar{u}$ tl èdung?

32. Gyā gu tl wahdas gu tl āknutila tlō-wons gien, gūt ga kiāgang-guns ging-an tl ëdung; Dalung un talung hüking-ang-gun, waigien gum dalung hyalthang-gun; talung kīlthdsū-

HA. 
gun, waigien gum dalung saigang-gun, hin tl shugung-gung; āū tliku wēt haade ishin ēdung.

33. John Baptist katlagundlu, gum la ga tā-ung-gīni, wine ishin gum il nịlthang-gīni ; waigien hants dāung-a la $\bar{a}$ ising dalung shugun.

34. Nung haada Git katlas gien, il lthdanūgung-gung; waigien nung ilthing-a kwotaga isgien, wine-nēlēga ishin, giashouganung-lēalung isgien ga daung-asgai tou-i ishin il ising dalung shugung!

35. Waigien nung kutung-as gitalung wautliwon il kutung-as kendagung-gung, hin Jesus shoun.

36. Waigien Pharisee haade swon la alth il lthdanīe da il gudang-an. W'aigien nung Pharisee haadas kwulth il kudsaian gien, lthdanūe un il kou-on.

37. Waigien ilnagai gu nung jada ijan dāung-aian; waigien nung Pharisee haadas gia nai ā il lthdanūs un il ūnshidalandlu, tō alabaster kulg à ijan il shkugang-an,

38. Waigien il stai sha il giāagan gien il sailthai-an, waigien il stai hung-ung hou alth il tîlthdiidan gien, kuts-ung alth lang-a il gīshouon, waigien il stai il shkwuntlaian gien, tō alth lang-a il tōdaian.

39. Waigien nung Pharisee haada la un aiang-an kang-andlu, hin il gudang-an, Anung 
ilthing-as prophetgasdlu, tliku nung jada et la ing-gu tl-dulthdas un il unshit-lthing-ãgan, il daung-as altha, hin il gudang-an.

40. Simon, dung ga gin shudie da di guldung-gung, waigien hin Jesus hanglthang il shudaian. Di ga lth shudi, nung itlagadas, waigien hin il shouon.

41. Waigien Jesus hin il shudaian, Nung giashildadalegga tl stung giashildadaian; nung swon pence hundredgai tlēlth shildaian, nung swon gia ishin tläale-tlēlthaian.

42. Tlistleuon gin alth la ga giashoulthinge gum kang-gang-andlu, il stung-won ga il dang-âwon. Gīnasū il kwōyädä-dsilthīishang? hin la il shudaian.

43. Nung gia kwon wau ga il dang-an il kwōyādädsilthīishang lth gudung-gung, hin Simon hanglthang shouon. Dung gū-yetdang; waigien hin la il shudaian.

44. Waigien nung jadas gwī il kīaian gien, hin Simon il shudaian, Dung kwulth lth kudsagundlu, gum di stai un untl di ga dung isdang-gun; la tlou hung-ung hou alth di stai tîlthildagun gien, kuts-ung alth il gīshūgun.

45. Gum di dung shkwuntlang-gun; la tlou lth kudsagun sta gum hawun tlan di stai shkwūuakung-gung.

46. Gum di kuts dung tôdang-gun; la tlou di stai tō alth tōdagun.

47. Althji alth il dãung-ie kwons-kianan 
lang-a ga gakung-ung dung ga lth shugung; di il kwōyād-euon-gun altha; lthing-an nung dāung-as gagakunsdlu tlou, althunis lthing an tl kwōyädagung-gung, hiı Simon il shudaian.

48. Dung dâung-as gagakung-gung, waigien hin nung jadas il shudaian.

49. Gishdu tl däung-as gakung-gung ? waigien hin ga lthdanāwon-gai gudang-an.

50. Dung yetdie $\bar{u}$ dung kogundang; gudung-ai dlaia alth lth kaid, waigien hin nung jadas il shudaian.

\section{Chapter 8.}

WAIGIEN hawidan wau dlă lana isgien lana knutila ai ishin il kāwong-an, waigien tla geu gu il gushus gien, Shanungitlagadas gia kingdomgai alth gialthing-e las alth tla ga il gialthindaian, waigien ga tlāalth wau gu stuns-gai la alth ijan.

2. Tl jāada ilthi hants dāung-a sta isgien sti sta ishin koganan, Mary Magdaleue hin kyāgan nung sta hants dāung-a jikwau ijan isgien,

3. Joanna Herod gia nai duman kang-lē Chusa hin kyāgan jā ishin, Susanna ishin, tl kulat kwon ishin, la alth isāwon, waigien gin il dāawos alth la da il tlāadūgang-an.

4. Waigien tlistleuon tl shkūl-euon gudā ijan gien, lana wautliwon sta ishin la un istlagandlu, tla ga il kègang-an ; 
5. Nung tutl-dsālēga tutldsã un kāgwaulan; waigien il tldsagundān, tīch keu-e in kūgaian; waigien tl tutldang-an gien, siet hetit is $\bar{u}$ tagan.

6. Waigien tīch ishin tĩjai ing-gut kügaian ; waigien īnastlaiandlu, hyilgälan, gum tîlthangani altha.

7. Tìch ishin gin lthkai ki shü-wīt kugaian; waigien gin lthkai kis wau alth inastlaian gien, $\bar{u}$ kou-hyistlaian.

8. Tich ishin kōi lä ai knūgaian gien, ĩuastlaian, waigien wau gut hundred-gagang-an, hin il shouon. Waigien hawun ashgai il shudagundān, Gin gudung-ai un nung geu-wos lth akwon il gudunda, hin kwūnan il shouon.

9. Waigien lagia disciplesgai tliku adshi kēgang-ai etgai da la alth kiānang-an.

10. Waigien hin il shouon, Shanung-itlagadas gia kingdomgai salgagai un unshidai dalung ga isdiāgung; tl kulat tlou kēgang. alth swonan un ünshidung; gin tl kens-kianan gum tl keng-uns gien, gin tl gudunsdlu ishin gum un tl unshidang-ai una.

11. Hinū kēgang-ai èdung; Malthē ù Shanung-itlagadas kil ijung.

12. Keu-e in ga isgai $\bar{u}$ Shanung-itlagadas kil gudung-gung; itan hetgwaulana katlas gien, tl gudung-ai sta kil il isdagung-gung, gum tl yetdans gien ishin gum tl kogun-angai unna. 
13. Waigien tījai ing-gu ga isgai ū tlistleuon kil tl gudunsdlu, gudung-ai lã alth tl isdagung-gung; althsgai ù gum shkūshang-wauung-gung, waigien kwai-umdsiwon tl yetdagung-gung, waigien tlistleuon tl edē tl kilkensdlu tl dl-dul-dung-gung.

14. Waigien gin lthkai kis shūwī ga kn̄īgasgai $\tilde{u}$ guduns gien, hawun tl istiidund̄an gudan lthkwīda isgien, ginlthing-a ishin, adshi henung-gai gudung-ai ligai ishin $\bar{u}$ kou-hyistlagung-gung, waigien gum wau gut ānē ālinstlagung-ung-gung.

15. Waigien kōi lā à ga isgai $\bar{u}$ gudung-ai yã alth isgien, gudung-ai lā alth ishin, kil guduns gien, duman $\bar{u}$ keng-euon-gung, waigien gudung-ai tladsga alth ān tl inastldagunggung.

16. Waigien nung haada kasodou ōgulthdasdlu, gum këlthga hetgwīi il isdagung-ung-gung, ishin gum tīdan hetgu il isdagung-ung-gung; kasodou keusi ing-gu tlou il isdagung-gung, ai ga isdsasgai wantliwon otgagai kang-gai una.

17. Gin saldas wautliwon kang-gēlthses altha; waigien gin salgas wautliwon un tl ūushits gien, ishin otgagai $\bar{a}$ isisang.

18. Althji alth lth tliku gin dalung guduns duman keng-ū ; gin nung dāas ga gin isdiāases altha; waigien gum gin nung dãansdlu, gin il dāung il guduns-hun la sta isdiāashang, waigien hin il shouon. 
19. Waigien il ou isgien il dōnalung ishin la un istlagan, waigien tl shkūlas alth gum tliku la à il et-waulthing-e kang-gang-an.

20. Dung ou isgien, dung dōnalung ishin kyā tjjūgiang-gung, waigien dung kang-gai da il gudung-wong, waigien hin la ga tl shouon.

21. Shanung-itlagadas kil ga guduns gien, ging-an tl ets $\bar{u}$ di ou isgien, di donalung ishin ijung, waigien hin hanglthang tla il shudaian.

22. Waigien althsgai sing-ai swon gut lã isgien, lagia disciplesgai ishin, kontlū gwē istligan; Shü-ē telg talung tlü-kātusdsun, waigien hin la il shudāwon; waigien il thūkaidāwon.

23. Waigien il hū-istalūgun-dan il kotīgłn; waigien shū-e $\vec{a}$ tudsou otōtalan; waigien otan wau kwon-ilths gien, il hastl-dalāwon.

24. Waigien la un il istla-āwon gien, lā il tla-shkināwon, Nung Itlagadas, nung Itlagadas, itil gudalgung, waigien hin il shuäwon. Waigien il shkinaian gien, tudsue isgien aiawe ishin il stidaian; waigien gü-ëlan gien, dlaiadan.

25. Gitlanu dalung yetdie ijung? waigien hin la il shudāwon. Waigien il lthwaugāwon gien il kuldung-āwon, Gīshdu à ijung, tudsue isgien aiawe ishin il shudas la kilth ging-an èdung? waigien hin gu il shudāwon.

26. Waigien Gerasene haade gia tligai, Galilee hanltha ijan, gu il tlū-katla-äwon. 
27. Waigien tlistleuon il kāalandlu, nung illthing-a à hants dāung-a ijan ilnagai sta la un katlagan; waigien jīng-a gum gin giandalthing-a il giandang-an, ishin gum nuh à il nāang-an, shalthung-nai ā tlou il nāgang-an. 28. Waigien tlistleuon Jesus il kang-andlu, il sailthaian gien, la hetgu il tīgūstlū-aian, Gūshāku di un dung katlang, Jesus, Shanungitlagadas sha āgwi isis Git $\bar{u}$ dung ijung? Gum di dung whaugung-dang-ai da dung alth lth kianung-gung, waigien hin kwūnan il shouon.

29. Hants gum shkwunans nung ilthing-as sta il kil-kāgwulthāgan altha. Nung hants dāung-as il gits-gilthdagigang-an altha; waigien la $\bar{a}$ tl lthotsagang-an, waigien stakwunsgulthda isgien yads alth ishin la tl kēchgīwaudagang-an; waigien gin alth il kēchgurwos ai il hyitidang-an, waigien hants dāung-as tligunltha à il ging-kāgang-an.

30. Gushinū dung kyang? waigien hin Jesus la alth kianang-an. Legion, waigien hin il shouon; hants dāung-a kwon la ai ijan altha.

31. Waigien gum hetgwaugai ai tla il kil-istiidang-ai da la alth tl kianang-an.

32. Waigien gaigu gwoshou kwon tldāuē ing-gut lthdanū-whūndalgang-an ; waigien gwoshougai kālthi ai tla il kil-itsgai da la alth tl kianang-an. Waigien il lādaian, 
33. Waigien hants dāung-as anung ilthingas sta is gien, gwoshou-gai kālthi ai ijan ; waigien gwoshou-gai stulai hunsta kuūstalan gien, shu-e ai kzūjang-gaian, waigien okētūgaian.

34. Waigien tlistleuon gwoshou-gai ga lthdanü-dagang-an-gai tliku gin ets kangandlu, tl kūstaian, waigien ilnagai gu isgien, ilnagai kiāgust ishin unsta tl shouon.

35. Waigien tliku gin edan tl keng-tlāwaugan; waigien Jesus un tl istlagan, waigien nung ilthing-a hants dāung-a sta ijan gin giandēlan gien, kudung-ëlan gien, Jesus hetgu kou-os tl kang-an; waigien tl lthwaugalan.

36. Waigien tliku hants dāung-a nung $\bar{a}$ ijan ligalan ga kang-an-gai tla ga shudaian.

37. Waigien Gerasene hāade gia tligai gu ga ijan wautliwon tla sta il itsgai da la alth tl kianang-an; tl lthwaug-euon-an altha; waigien kontlū gwë il katlīgan gien, il stēlan.

38. Hants dāung-a nung sta ijan tlou la alth itsgai da la alth il kianang-an; waikian la il kilkaidan,

39. Gyang nai gwi lth stîlth, waigien tliku Shanung-itlagadas gin euon dung un isdagun unsta Ith shū, waigien hin il shouon. Waigien il kaidan gien, ilnagai ashkwau-hun gut tliku gin euon Jesus la un isdaian alth il gialthindaian.

40. Waigien Jesus silthgang stēlan gien, ga shkūlasgai la alth gudung-ai lāgan; la keu tl et-ôdsou-on altha. 
41. Waigien nung îlthing-a Jairus hin kyāgan katlagan, synagogue-nagai un il itlagadāgan; waigien Jesus hetgu il tīgūstlü-aian gien, lagia nai ai il kudsie da la alth il kian. ang-an ;

42. Il gũjang-a swonshang-an da tuda tlāalth wau gu stang-an kotulthdalan altha. Waigien à Jesus kāgandlu, la odō gu tl hyitsgut-euon-an.

43. Waigien nung jada tuda tläalth waugu stung lthū i sta aian, waigien gin il dägan wautliwon tla-hyildālẽ ga il isdaian gien, gum tliku la tl tl-ligal-lthing-e kang-gang-an,

44. La dsiga katlagan, waigien gin il giandaian kiai il gitsgilthdaian; waigien hawīdan il lthu ì sta aian tlan-ēlan.

45. Gìshdū di tl-tlang? waigien hin Jesus shou-on. Waigien tl wautliwon ga kudaiandlu, Nung Itlagadas, tl shkūlas dung odō gu hyitsgidung, hin Peter isgien la alth ga ijan ishin shouon.

46. Di nung tl-tlagun, dugwia di sta ijun un di inshidun altha, waigien hin Jesus shouon.

47. Waigien tlistleuon nung jadas gum salgans kang-andlu, il katlagan gien, il dlīdaian gien, la hetgu il tīgūstlī-aian, waigien tl haada wautliwon hang-gu gin agunan la il tl-tlaian gien, tliku hawidan il ligalan ishin unsta il shouron.

48. Di gūjang-a isis, dung yetdas $\bar{u}$ dung 
tl-ligulgung; gudung-ai dlaia alth lth kaid, waigien hin la il shudaian.

49. Hawun il gushugundān, synagogue-nagai un nung itlagadas gia nai sta nung katlagan, Dung gujjang-a kotulgung; gum lth nung Itlagadas gudung-ai lthkwīdadang, waigien hin il shouon.

50. Waigien Jesus gudang-andlu, Gum lth lthwaugang; hing-an lth yetdi, waigien il ligulthshang, hin hanglthang il shudaian.

51. Waigien tlistleuon nai un il katlagandlu, gum nung haada kotdung ai il kudsadang-an, tlan Peter isgien, John ishin, James ishin, waigien nung ä-jadas hāt ishin, waigien il ou hunisun kotdung ai il isdsadaian.

52. Waigien tl wautliwon saigaian gien, tl kilthdsāwon; Gum lth saigang- $\bar{u}$, gum il kotulthans altha, lā $\overline{\mathrm{u}}$ kotdang, waigien hin il shouon.

53. Waigien la un tl kah-euon-an, il kotalan un tl unshidan altha.

54. Waigien il stlai lang-a il gitsgilthdaian gien, la ga il kiāgang-an, Nung à-jadas, kālthūltha, waigien hin il shouon.

55. Waigien il althandai la gwī stēlan gien, hawidan il kālthā-won; waigien gin tālthing-e la ga il kil-isdaian.

56. Waigien il yāalung kuldung-ägan; waigien tliku gin edan gum nung swonshung-gahun la il shudalthang-āwon. 


\section{Chapter 9.}

WAIGIEN ga tlāalth wau gu stuns-gai un il ai-ang-an, waigien hants dāung-a wautliwon un isgien, sti tla-ngistlie un ishin dugwīa la ga il isdāwon.

2. Waigien Shanung-itlagadas gia kingdomgai alth tla geu gu il gushū-awe un isgien, tl stis il tla-ngistlawwe un ishin, la il kil-istiidäwon.

3. Waigien liin la il shudāwon, Dalung istīidsdlu lth gum gin isdang-wong, tushka isgien, gwau-alth ishin, shibli ishin, dāla ishin lth gum isdang-wong; lōdats chi-stung ishin lth gum isdang-wong.

4. Waigien nuh ai dalung isdsasdlu lth, sta dalung istîidai keu à nā-ung-wong.

5. Waigien gum dalung $t \mathrm{l}$ isdsadansdlu, tlistleuon ilnagai sta tlang-a dalung istiidsdlu lth, stang sta kōi-e giidwong, tla hanltha witnessgagai una, waigien hin la il shudāion.

6. Waigien il istīidāwon, waigien lana ai il istalgung-āwon gien, gialthang las alth tla geu gu il gushu-âwon, waigien tlìian tla il tlligulthūgang-an.

7. Waigien Herod nung tetrarch-gas tliku gin edan wautliwon gudang-an; waigien il gudung-ai lthkwīd-euon-an, John kotal sta kālthū-ung tl ìlthi shouon altha;

8. Elijah tl kang-ung tl îlthi ishin shouon; 
tlidlu ga prophetgagan swon kālthū-ung tl kulat ishin shouon.

9. John kuts wau sta lth shkiketlagun; gishd edē tlon hin gin et lth gudung-gung? waigien hin Herod shouon. Waigien il kanggai da la il gudang-an.

10. Waigien ga apostlesgasgai tlistleuon silthgang stēlandlu, tliku gin il edāwon la ga il shudāwon. Waigien la il isdāwon gien, lana Bethsaida hin kyāgan a la alth il kaidāwon.

11. Waigien ga shkūlasgai un ünshidalandlu, la dla tl istiidan; waigien tla alth il gudung-ai lagan gien, Shanung-itlagadas gia kingdomgai alth tla à il gushonon, waigien ligulai da ga gudunsgai il tl-ligalan.

12. Waigien sing-i-aian; waigien ga tlaalth wangu stunsgai istlagan gien, Ga shkūlasgai lth kil-istiid, waigien lana isgien, tliga odō is ishin, $\bar{a}$ tl istīids gien, gu nuh $\bar{a}$ tl jsis gien, tou tl isdie una; adlun tligunltha talung isis altha, waigien hin la il shudāwon.

13. Dalung lth tl lthdanūdū, waigien hin la il shudāwon. Shibli kū-tlēlth swonan talung dāung, shkolthung stung ishin; tla un tou talung dahwosdlu tlon, tla dlū èt-lthing-āgung, waigien hin il shu-âwon.

14. Tl ilthansida thousandgai tlētth dlu edan altha. Tläale tlēlth $\bar{a}$ lth gut alth kil-tlā-ùdalü, waigien hin gyang disciplesgai il shudaian. 
15. Waigien ging-an il wau-āwon gien, tl wautliwon il tlā-ū-dāwon.

16. Waigien shibligai kū tlēlth isgien, shkolthung-ai stung ishin il isdaian, waigien shi ga il kēilthaian gien sing il kil-lāgan, waigien ai il ginanang-an ; waigien gyang disciplesgasgai ga il isdaian, ga shkūlasgai hetgu il isdāawe una.

17. Waigien tl lthdanāwon gien, tl skisūōdsou-on; waigien kwōkung-awe wau silīa ijan gudā tl isdaian gien, basket a-tlāalth wau gu a-stung sta-hāwon.

18. Waigien la swonan singlthgang gushouondlu, lagia disciplesgai la alth ijan; Gìshdū di ising ga shkūlasgai shugung ? waigien hin la alth il kianung-āwon.

19. John nung Baptistgas-a; tl ilthi ishin Elijah dung ising shugung; waigien tl ilthi ishin tlidlu ga prophetgagan-gai swon kālthūs dung ising shugung, waigien hin hanglthang il shūāwon.

20. Gisshd tlou di ising dalung shugung, waigien hin la il shudāwon. Shanung-itlagadas gia Christgai $\bar{u}$ dung ijung, waigien hin Peter hanglthang shouon.

21. Waigien la il stidāwon gien, gum nung haada ga adshi la il shūdalthang-āwon;

22. Waigien hin il shouon, Nung haada Git gin shing-idsa kwon āndung-ashang, waigien ga kēasgai isgien, liplēt un ga itlagadasgai 
ishin, tukālung-lē-alung ishin la ga gwou-wauashang, waigien la tl tīāashang, waigien shin lthonulthsdlu il kālthū-ashang, hin il shouon.

23. Waigien hin ishin tl wautliwon il shudaian, Di dlā kagai da nung gudunsdlu, agung il chāa-gudang-das gien, shantlan wautliwon shkaul-a-magai ung-a il shkeulthgaguns gien, di dlā il kagai ū lagung.

24. Hēnung-gang kogundie da nung gudunsdlu, il gūdāases altha; di agunan hēnunggang nung gudasdlu tlou, ung-a il kogundāashang.

25. Gūshū nung haada giwī kāalgung, hēttligai ashkwau-hun la gwi kāalths gien, agung il gudasdlu-a?

26. Di alth isgien, di kil alth ishin, nung êdasdilu, la alth ishin nung haada Git èdāashang, tlistleuon gyang unyägudung-gai isgien, nung Aung-as unyāgudung-gai ishin, angels las unyāgudung-gai dung-alth ishin, il katlasdlu-a.

27. Waigien yenkien dalung ga lth shugung, Adlun ga tljūgians îlthi, Shanungitlagadas gia kingdomgai kang-gai keu, gum kotulthga-swonung-ung-ashang, hin tl wautliwon il shudaian.

28. Waigien adshi il shudaian sta shin stanshang-a èlandlu, Peter isgien, John ishin, James ishin il isdaian, waigien singlthgang il gushue un tldāwe gwīi il kulthaian. 
29. Waigien hawun singlthgang il gushugundān, il hung-i āda èlan, waigien gin il giandaian otgālan gien, à dsū-i-hung-i-āgan.

30. Waigien tl îlthansida stung la à gushouon, Moses isgien Elijah ishin $\bar{u}$ ijan;

31. La unyāgudung-āwos kang-gas gien, Jerusalem gu il kotalang-kasas edē il kāūgāivon.

32. Waigien Peter isgien, la alth tl ijan ishin, kung-ä-euonan; waigien tlistleuon il shkin-jēlthā-wondlu, la un yagudung-as isgien, tl ilthansida stung la kwulth tljūgians ishin il keng-awon.

33. Waigien la sta il istīidūgundān, hin Peter Jesus shudaian, Nung Itlagadas, adlun talung itsgai $\bar{u}$ lāgung; giang-ū-nai tī-lthonulth talung tloulthadsun; tî-swon dung un isgien, tī-swon Moses un ishin, 1̄i-swon ishin Elijah un isisang, hin il shouon; waigien gum tliku il shūs un il ūnshidang-an.

34. Waigien hawun adshi il shudagundān, yanung-ēlan gien, la ga saldāwon; waigien yanung-ai ai il isāwondlu, il lthwaugāwon.

35. Waigien yanai sta kil ijan, Di Git $\overline{\mathrm{u}} \overline{\mathrm{a}}$ ijung, nung lth gwūlas-a ; il kil lth gudung- $\bar{u}$, waigien hin kil shouon.

36. Waigien tlistleuon kil ijandlu, Jesus swonan gu ijan. Waigien il kwūlgundāwon gien, gin il keng-āwon wautliwon gai nūt gum nung hãada ga il shudang-āwon. 
37. Waigien waudālēkw tldāive sta il istallthāwondlu, tl shkūl-euon la un istlagan.

38. Waigien nung ilthing-a shkūlie shu sta kiā-gang-an gien hin il shouon, Nung Itlagadas, di git dung kang-gai da dung alth lth kianung-gung; tlan di git swonshuns il isis altha;

39. Waigien hants $\overline{\mathrm{u}} \mathrm{il}$ isdas gien, umlthistan il kung-adagung-gung; waigien la il ging-kēù-nuns gien, il helthi ai sta sōlū ijung-gung ; waigien la sta kāstie-jīng-as gien, ishin ung il kedligadang-euon-gung-gung.

40. Waigien wausta tl dang-gai da dung-gia disciplesgai alth lth kianung-gun; waigien a il dsigî-ui-gun, hin il shonon.

41. Gūdista kang-gai gum yetdang isgien, genjia ishin dalung isis, gisdlu dalung kwulth lth isis gien, dalung keu lth etishang? althgwi lth gitang isdi, waigien hin Jesus hanglthang shouon.

42. Waigien hawun il kāgundān, hants dāung-as il tl-dl-dulthdaian gien, il kē-ñnung-euonan. Waigien Jesus hants gum slıkwunans stidaian gien, nung ilthing-a kntsuis il tl-ligalan, waigien aung-ung ga silthgang la il isdaian.

43. Waigien Shanung-itlagadas unyāgutdung-gai un tl kuldung-̄a-ōdsou-on. Waigien gin wautliwon il isdaian hawun tl wautliwon unkuldung-āgundān, hin gyang disciplesgai il shudaian,

HA. 
44. Adshi gushue ai duman dalung geuying-ai lāgung; nung haada Git tl haada stlai ai tl isdāases altha, hin la il shudāwon.

45. Waigien adshi gūshūe un gum il ūnshidang-āwon, waigien gum un il īnshidang-awe un la sta salgāawon; waigien adshi gushue tliku etgai da la alth kianung-ai ga il lthwaugāwon.

46. Waigien la shu nung itlagadawau-ases edē il kāūgìdāwon.

47. Waigien gin edē il kwulthilthāwon Jesus kang-andlu, nung a-krutsū il isdaian gien, kwulthgang la il isdaian, waigien hin la il shudāwon,

48. Nung-hun anung ā-kutsūis di kyē agunan isdasdlu, di $\bar{u}$ il isdagung-gung; waigien nunghun di isdasdlu, di nung kil-kaidun $\bar{u}$ il josdagung-gung; dalung wautliwon hang-ast nung ets itlagadas altha, hin la il shudāwon.

49. Nung Itlagadus, dung kyē alth hants dāung-a tla sta nung dans talung keng-gun, waigien la talung stidagun, gum itil alth il stung-uns altha, waigien hin John hanglthang: shouon.

50. Gum lth il stidang-īi ; gum dalung hanltha nung edansdlu, dalung gūst il ets altha, waigien hin Jesus il shudaian.

51. Waigien tlistlewon shi ga la tl isda-ungkasas shantlanai waudlu èlthdalandlu, Jerusalem ā il kogai un il gudung-ai elthgīgan, 
52. Waigien kōnastgang gītsadalung il kilistriidan; waigien il istīidāwon gien, Samaria haade gia ilnagai swon ai il isdsäawon, la keu gin il èlthgīdawe una.

53. Waigien la ga tl gwou-aian, Jerusalem à kagai un il gudung-ai elthgigan altha.

54. Waigien tlistleuon James isgien John ishin lagia disciplesgai ijan adshi kang-andlu, Shalana, sha-tligai sta das talung kil-is gien, tl ōgulai da gu dung gudung? hin il shuāwon.

55. Waigien la gwĩ il kēi-āwon gien, la il stidāwon.

56. Waigien lana kulat ā il istiidāwon.

57. Waigien hawun keu-e gūt il istalügundān, Tlijan ga-hun dung kas ging-an dung dlä lth kàashang, hin nung ilthing-a swonshung il shudaian.

58. Nukudsĩ ū nēē dāung, siet hetit is ishin lthtelg dāung, nung haada Git tlou gum tlijan il kuts kē-ū-dalthing-e dā-ung-gung, waigien hin Jesus il shudaian.

59. Di dlä lth kā, waigien hin Jesus nung kulat shudaian. Shalana, kwai lth aung-ung di lthēa-wau-tlagang-di, waigien hin il shouon.

60. Ga kotulthgiasgai lth akwon ga kotulthgiasgai ung-a lthēôda, da tlou Shanung-itlagadas gia kingdomgai tla ga shudãagai lāgung, waigien hin la il shudaian.

61. Dung dlä lth kāashang, Shalana; kwai 
lth giagun nai à ga isgai à di kil-lā-tlagang-di, waigien hin nung kulat ishin shouon.

62. Nung hāada tliga-uldsalawe gitsgīlthdas gien, silthgang il kensdlu, gum Shanung-itlagadas gia kingdomgai un il lā-ung-gung, waigien Jesus hin il shudaian.

\section{Chapter 10.}

TY AIGIEN adshi dlā Shalana ga kulat tlāale jikwau kenstaian, waigien lana wautliwon isgien, tliga wautliwon ai ishin il kāwongung-kasas ā stung-ā kōnastgang il kilistiidāngan.

2. Waigien hin tla il shudaian, Tukwūlāenon-gung, ga lthong-gwilasgai tlou gū-kutsūgung; althji alth lth tukwūlā un nung Itlagadas alth kianung-ī, tukwīlas à ung-a tla il kilistiidai una.

3. Istiidūltha; ōch shū-wī lumado tl istiidaguns ging-an ū dalung lth isdang.

4. Dala gwau-ale isgien, āltliga ishin, stushgaga ishin lth gum isdang-wong; waigien dalung istalsdlu lth gum nung haada à kil-1īung-wong.

5. Waigien tliku nuh et ai-hun dalung isdeasdlı, Akwon lth gudung-ai dlaia adshi nai à isdi, hin lth shu-tlagang-wong.

6. Waigien nung gudung-ai dlaia git gu isisdlu, dalung gudung-ai dlai-e la ing-gu isi- 
sang; gum gu il isansdlu tlou, dalung gwi silthgang stilthishang.

7. Waigien gai nai à swonan Ith iswong, waigien dalung ga gin tl isdas ging-an lth tawong; nung lthong-gwilasdlu, giashougai unga il isdie las altha. Gum lth gut giāgūst nuh ai istalung-wong.

8. Waigien tliku lana et ai-hun dalung isdsas gien, dalung tl isdsadasdlu, dalung hetgu gin tl isdas ging-an lth tâ-Iwong;

9. Tl sti gu isisdlu lth, tl-ligulthwong, Shanung-itlagadas gia kingdomgai dalung un āanēl-gung, waigien lth hin tl shudawong.

10. Waigien tliku lana et ai-hun dalung isdsas gien, dalung ga tl gwou-osdlu lth, keue à isāwong,

11. Waigien lth hin shū-wong, Dalung-gia ilnagai kōiē hun itil stai ga dsilthguts dalung hanltha etgai un wausta talung gīshūgung; waigien Shanung-itlagadas gia kingdomgai aanēlths un tl lth ung ünshitwong, hin lth shū-wong.

12. Gai shantlanai ai Sodom ilnagai un shing-idsas telg, gai ilnagai un shing-idsãashang dalung ga lth shugung.

13. Chorazin, dung un gin ga lthwaugigàashang! Bethsaida, dung un gin ga lthwaugīgāashang! dalung shu lthong-gwila euonda ijun ging-an Tyre isgien Sidon gu ishin ijasdlu, tlidleu-un tl gudung-ai stilths gien, whansda 
tl giandîlths gien, aiid à tl kouons-gaia-hunggung.

14. Waigien tla tl ging-kilisalunsdlu, Tyre isgien Sidon un ishin shing-idsas telg dalung un shing.idsāashang.

15. Waigien dā ishin, Capernaum, shatligai à gu dung edalt] ãash? hët ga Hades ā dung tl isdāashang.

16. Dalung kil nung gudunsdlı, di kil ū il gudung-gung; waigien dalung ga nung gwouosdlu, di ga $\bar{u}$ il gwou-gung; waigien di ga nung gwou-osdlu, di nung kilkaidun ga ù il gwou-gung, waigien hin tla il shudaian.

17. Waigien ga tlāale jikwausgai gudan lā alth silthgang stēlan, Shalana, hants dāung-as hun dung kyē agunan itil kilth ging-an èdung, waigien hin il shu-âwon.

18. Waigien hin la il shudāwon, Sē-à-ūlthda ging-an sha-tligai sta Satan dlū-is lth kenggun.

19. Sik isgien, scorpion ishin ing-gu tudul dungai un isgien, dalung telg nung kens gia dugwigai wautliwon telg ishin dalung lth tldugwiāgun; waigien gum gin hun dalung un dāung-ung-ashang.

20. Hants dalung kilth ging-an ets alth lth gum gudung-ung lādang-ū; sha-tligai gu dalung kyē kälung-as alth tl lth gudung-ung lādū, waigien hin la il shudāwon.

21. Gai ashkosbgadai gut Hants Las alth il 
gudung-ai lāgan, waigien hin il shouon, Aunga, sht-tligai isgien hēt-tligai ishin un nung Itlagadas, ga kutung-asgai isgien, gin un ga ünshitsgai ishin sta adshi dung salgun gien, tl ākutila dung kendagun sing dung à lth killāgung; āng, Aung-a; $\bar{a} \bar{u}$ tliku etgai dung givillagun altha.

22. Gin wautliwon di Aung di ga isdagun; waigien gum nung-hun nung Git-as un inshidang-gung, tlan il Aung swon-an-a; waigien gum nung-hun nung Aung-as un ūnshidanggung, tlan il Git swonan la un ūnshidung, waigien nung-hun nung Git-as il kendas $\overline{\mathrm{u}}$ la un ūnshidung, hin il shouon.

23. Waigien gyang disciplesgai gwī il kēaian gien, hin il shū-kwolthdaian, Gin dalung kens ga kensgai ũ lãgung;

24. Ga prophetga kwon isgien, ga king-ga kwon ishin, gin dalung kens kang-gai da gudang-an, waigien gum tl keng-ang-an; waigien gin dalung guduns gudung-ai da tl gudang-an, waigien gum tl gudung-ang-an, hin il shouon.

25. Waigien nung king-gogung-lēga giāagan gien, il edē il kilkang-an, Nung Itlagadas, di hēnung-a-swonung-ai un gūshū lth isdāashang? waigien hin il shouon.

26. Gushinu king-go-gung-ai ëdung? Gushinu dung lidida-tljū-ūjung? waigien hin la il shudaian. 
27. Waigien hin hanglthang il shouon, Gudung-ung shashkwau-hun alth isgien, althandang dlishkwau-hun alth ishin, dugwigang wautliwon alth ishin, kwulthilang wautliwon alth ishin, Shalana Shanung-itlagadas dung kwō-yādie lāgung; waigien ltbū-ung dung kwōyādas ging-an dung kwulth-it tl nāans dung kwōyādie lagung, hin hanglthang il shouon.

28. Dung kil-lthyetdang; ging-an lth et, waigien dung hēnung-āashanğ, waigien hin la il shudaian.

29. Il las tlou il kendie da il gudang-andlu, Gīshdin di kwulth nung nas ijung? hin Jesus il shudaian.

30. Waigien Jesus hin hanglthang shouon, Nung ilthing-a Jerusalem sta Jericho ã kāālan; waigien kwolthdālē-alung un il katlagan, waigien gin il giandas la sta tl isdas gien, la tl shkidang-an, waigien il kotulthdalsdlu la sta tl istīidan.

31. Waudlu nung liplētga gai keu-e gut kāalan; waigien tlistleuon la il kang-andlu, la hanlthīt il kā-swonang-an.

32. Waigien nung Levite-ga ishin ging-an ijan, tlistleuon un il katlagan gien, la il kangandlu, la hanlthīt il kā-swonang-an.

33. Waigien nung Samaria hāada hawun kāgundān, giāgu il is un il katlagan; waigien tlistleuon la il kang-andlu, la kalthshint il gwaulan. 
34. Waigien la un il katlagan, waigien il kosilas gwī tō isgien wineishin il giadāwon gien, lang-a il kiāwon; waigien gyang gin tīigagai gwau la il koudujan, waigien lthdanou-nai ai la il isdaian gien, duman la il kang-an.

35. Waigien waudālēkw penny kēstung il isdaian gien, nung nuh-lēgas ga il isdaian gien, hin il shouon, Duman lth il keng-ung; ashgai telg lē gin dung bīlūdasdlu, tlistleuon silthgang lth katlasdlu, dung ga lth shou-ashang, lin il shonon.

36. Kwolthdālē-alung un nung katlagan un ga lthonulth istlagan-gai gīnasu la un kunggudung-aiang dung gudung-gung ? waigien hin Jesus il shudaian.

37. La kalthshint nung gudang-an-a, waigien hin il shouon. Kaidltha, waigien lth ging-an wau, waigien hin Jesus il shudaian.

38. IVaigien hawun il istalīgundān, lana a il isdsāwon; waigien nung jada Martha hin kyāgan kwulthgang il isdaian.

39. Waigien dōn il dāgan, Mary hin il kyāgan; althunis Shalana hetgu kou-aian gien il kil lang-a il gudang-an.

40. Martha tlou lthong-gwil-euon-an gien, il gudung-ai lthkwīdaian; waigien la un il katlagan gien, Shalana, di dōn di swonan lthong-gwildas gu gum alth dung ai-ī-angūsh ? di da lth il tlāadalth, hin il shouon.

41. Waigien Shalana hin hanglthang il shu- 
daian, Martha, Martha, gin kwon alth dung gudung-ai lthkwīdang.

42. Gin swonshung swonan gu stalthīgāgung; Mary gin las isdagun altha, waigien gum la sta isdiang-ashang, hin la il shudaian.

\section{Chapter 11.}

W AIGIEN tliga swonshung gu singlthgang il gushouon; waigien tlistleuon tlan il shouondlu, Shalana, singlthgang gushou lth itil shkotadi, John gyang disciplesgai gin shkotadagun ging-an-a, hin lagia disciplesgai swon il shudaian.

2. Waigien hin la il shudāwon, Singlthgang dalung gushusdlu lth hin shūū, Aung-a, akwon lth kyang kwoyãdi. Akwon lth kingdom-gai althg" i ga ung-a isdi.

3. Shantlan wautliwon gut lth sing gut tou itil ga isdi.

4. Waigien lth itil dāung-as kalthshint gudung; itil un ga dāung-asgai kalthshint talung gudung-gung-us altha. Waigien lth gum edē kil-kang $\bar{a}$ itil istaldang, hin la il shudāwon.

5. Waigien hin ishin la il shudāwon, Gīshdū dalung shu tō dāung? waigien aliako la à il kaids gien, hin la il shudalthing-āgung, Di tō-i, shibli kū-lthōnulth lth di shilda-di;

6. Di tô-alung swon tliga jīng-a sta di un 
katlas altha, waigien gum gin la lth tādalthing-e kang-gang-gung, hin la il shudalthing-āgung; ;

7. Gum lth di gudung-ai kil-lthkwīdang; keu-e wau shkotajāgung, waigien gitalung dung-alth lth tidang; gum tliku dung ga isdie un lth kālthoulthing-e kang-gang-gung, waigien anā nung is hin hanglthang shoulthingàgung.

8. Il tō-i il isis alth $\bar{u}$, il kālthūe isgien, la ga isdie ishin ga il gwou-os-kianan, la alth il kianung-hunsguts alth $\bar{u}$ il kālthūs gien, tlistleu ga il stalthasi ging-an la ga il isdāashang dalung ga lth shugung.

9. Waigien hin ishin dalung ga lth shugung, Kianung-ūltha, waigien dalung ga isdiāashang; da lth keng- $\bar{u}$, waigien dalung kēi-āashang; shkusdagā-ūltha, waigien dalung un wau sta ostlāashang.

10. Tl wautliwon hun gin da kiānuns $\bar{u}$ isdagung-gung; waigien gin da nung kens $\overline{\mathrm{u}}$ $\bar{u}$ këi-gung-gung; waigien nung shkusdagasi un wausta ostlāashang.

11. Dalung swon aung-asdlu, waigien il git shibli knūga da la alth kiānunsdlu, kwau gu la ga il isdāash? shkolthung da ishin il kiānunsdlu, shkolthung shoutga gu sik la ga il isdāash?

12. Khou da ishin il kiānunsdlu, scorpion gu la ga il isdāash? 
13. Dalung dāung-askianan, gin lā gitalung ga isdie un dalung ūnshitsdlu, gīsdlu wau telg dalung Aung sha isis la alth ga kiänunsgai ga Hants Las isdāashang? hin tla il shudaian.

14. Waigien hants dāung-a nung à ijan kilgãwon wau sta il dang-an. Waigien tlistleuon hants dāung-as la sta ijandlu, nung kil-gāwon gushouon; wàigien ga shkn̄lasgai kuldung-ägan.

15. Beelzebub, hants dāung-as un nung itlagadas, alth hants dāung-as tla sta il danggung-gung, waigien hin tl ilthi shonon.

16. 'Il ilthi ishin il edē kil-kang-an gien, sha sta gin kang-īdsada da la alth tl kiānang-an.

17. Waigien tliku th kwīthilths un il ünshidan gien, hin tla il shudaian, Tliga wautliwon gu hun gut hanltha tl etsdlu, tl hīlīgung-gung; waigien nuh ti-swonshung $\bar{a}$ ga isgai gut hanltha ēlthsdlu, tl hīlūgung-gung.

18. Waigien Satan ishin hanlthalthgang ëlthsdlu, gushinu lagia kingdomgai dugwiatljū-ashang? Beelzebub alth hants dāung-as tla sta th dang-ung dalung shūs altha.

19. Waigien Beelzebub alth hants dāung-as tla sta lth dansdlu, gishd-alth-ī dalung gitalung ishin tla sta dang-gung-gung? althji alth $\overline{\mathrm{u}}$ dalung gitalung dalung un ging-kilisalang-lëgāashang.

20. Ltha tlou Shanung-itlagadas stlukung-i 
alth hants dāung-as tla sta dansdlu, Shanungitlagadas gia kingdomgai dalung ing-gu isilgung.

21. Tlistleuon nung ilthing-a dugwias tlāwatt-ānī ung-a gits-guts gien, nai duman ung-a il kensdlu, gin il dāas ū duman lang-a èdung;

22. Waigien tlistleuon la telg nung dugwir la un katlas gien, la il dukw-āadasdlu, tlāwanāni wautliwon alrh il kwauguts la sta il isdas gien, ginagai lang-a il tlāadīgung-gung.

23. Gum di alth nung isans $\bar{u}$ di hanltha èdung; waigien gum di alth gudā gin nung: isdāans $\bar{u} \bar{u}$ hagūjang-gung.

24. Nung hants gum shkwīnans nung îlthing-a sta isisdlu, tliga gu untl gū kalthgūt il kas gien, unshandsuda-danai da il kenggung-gung; waigien gum il kēi-ans gien, Gyang nai gwī lth stîlthshang, gyā sta lth ijun à-a, hin il shugung-gung.

25. Waigien tlistleuon il katlasdlu, lthkioudalas gien, duman gin thïishkutung-as ishin il keng-gung-gung.

26. Itan il kaids gien, hants la telg dāung-a jikwau il isdagung-gung; waigien ai tl isdsas gien $\bar{a}$ tl nāung-gung-gung; waigien nung: îlthing-as kwūuāil dāung-aian telg il dāungèdung-gung, hin tla il shudaian.

27. Waigien hawun adshi il shudagundān, nung jada shkūlie shu sta kilthgulaian, Dung nung dulthgang-an isgien, nung tlinū-e 
dung tlanan ishin yenkien lägung! waigien hin la il shudaian.

28. Ang, Shanung-itlagadas kil ga guduns gien, duman $\bar{u}$ kens tlou wautelg lāgung, waigien hin il shouon.

29. Waigien tlistleuon tl shkülas la à gudā ijandlu, hin il shū-īdan, Adshi gūdista kanggai güdista kang-gai dāung-a ijung; gin kangüdsada da tl keng-gung-gung; waigien gum gin kang-üdsada tla ga isdiang-ashang, tlan Jonah gia kang-üdsadagai swonan-a.

30. Nineveh haade un Jonah kang-ūdsēlan ging-an, nung haada Git ishin adshi gūdista kang-gai un etishang.

31. Ging-kilisalang shantlanai ai ousik haade un nung queen-gas adshi gūdista kanggai alth giāang-ashang, waigien tla ga ēdā il idāashang; tliga jīng-ā sta Solomon kutung-as kil gudung-ai un il katlagan altha; waigien Solomon telg nung ets adlun ijung.

32. Nineveh haade ishin ging-kilisalang shantlanai ai adshi gūdista kang-gai alth tljūgiāng-ashang, waigien êdā tla ga isdāashang; Jonah tla geu gu gushouondlu, gudung-ung tl stîlthdaian altha; waigien Jonah telg nung ets $\bar{u}$ adlun ijung.

33. Nung haada tlistlewon kāsōdou ōgulthdasdlu, lthē-ang kol ai isgien, kwīdou hetgwī ishin gum isdagung-ung-gung; keusi ing-gu tlou il isdagung-gung, ga isdsasgai otgagai ù kang-gai una. 
34. Dung lthī un ī dung hung-i kãsōdouwaugung; dung hungi lasdlu, dung lthū dlishkwau-hun ishin otga alth stāñgāashang; dung hung-i dā-ung-asdlu tlou, dung lthū ishin alga alth stāūgāgung.

35. Althji alth otga dung $\bar{a}$ is algagai lth keng.

36. Althji alth dung lthũ dlishkwau-hun otga alth stāūgas gien, gum lthing-an algāansdluyenkien otga alth stāūgäashang, kāsōdou ōgai otdasdlu dung ga otgāguns ging-an-a, hin il shouon.

37. Waigien hawun il gushugundān, nung. Pharisee haada kotdung il lthdanü-halan; waigien il kudsaian gien, lthdanue un il kouon.

38. Waigien il lthdanue konast gum il stlandlinang-an nung Pharisee haadas kang-andlu, il kuldung-āgan.

39. Waigien Shalana hin il shudaian, Dalung Pharisee haadas shkutlang-we kul isgien kēilthgagai kyagūstsi ishin tla-shkwūnagung-gung; dalung kalthi tlou kwolthda alth isgien, gin dāung-a alth ishin stāīgãgung.

40. Dalıng kōnuns, gum gu kyagūstsi nung tloulthaian nāgūstsi ishin tloulthang-ūja?

41. Nägūst gin is tl lth Shanung-itlagadas ga isdū; waigien gin wautliwon dalung un shkwūnagulgung.

42. Dalung Pharisee haadas un gin ga lth- 
wau-gīgāashang! mint isgien, rue ishin, gin inastlas wautliwon ishin tich Shanung-itlagadas ga dalung isdagung gung; ging-kilisalang isgien, Shanung-itlagadas kwōyālie ishin tlou da dalung kēshgìdung-gung; a gin isis dalung isdie lāgung, waigien ga kulat da dalung kēs':garlai tlou gum lā-ung-gung.

43. Dalung Pharisee haadas un gin ga lthwaugīgāashang! synagogue-nagai $\bar{a}$ aulthgahung-u tudsgwau is dalung knoyādas gien, gyāgu tl wahdasgai gu ishin dalung hung a tl kwahgulthdie ishin dalung kwōyālagung-ns altha.

44. Dalung un gin ga lthwaugigaaashang! tl ltl ēāwau gum kang-gans gien, tl haada ing-gut istalguns gien, gum wau un unshidung-uns ging-an dalung ets altha, hin la il shudaian.

45. Nung Itlagadas, hin dung shūsdlu, itil ishin dung kil-ēdāng-gung, waigien hin kinggogung-lëalung swon hanglthang il shudaian.

46. Waigien hin il shouon, Dalung kinggogung-lëgas un ishin gin ga lthwangigaaashang! gin ken-euon tl haada dalung giing-wūnidungus altha, dalung tlou guin stlukung-ung skaswonshung alth-hun tla da tlä-ēdung-unggung.

47. Dalung un gin ga lthwangigoasashang! gyagu ga prophetgas lthēāwos dalung tloulthas altha, waigien diiung aung-alıng $\overline{\mathrm{u}}$ tl tldaian. 48. Althji alth tliku dalung aung-alung 
waugan un dalung witness-gas gien, dalung gwūlāgung; tla tl tldas gien, dalung ishin tl lthēàwos tloulthas altha.

49. Althji alth $\bar{u}$ hin Shanung-itlagadas gia kutung-gai shugang, Ga prophetga isgien, ga apostlesga ishin tla ā lth kil-isisang; waigien tl ilthi tl tldas gien, ishin gin shing-idsa tl āndung-dāashang;

50. Het-tligai elthgigan sta ga prophetgasgai wautliwon ai wausta kwah-yandaian tāa adshi gūdista kang-gai tl tlū-unshkotdie una.

51. Abel ai wausta kwah-yandaian gien, Zachariah altargai isgien nuh kwōyas ishin yākosīa tl tiiiaian ai wausta kwah-yandaian ishin, tl kulat wautliwon ai wausta tl kwah-yandaian ishin tāa, adshi gudista kang-gai tl tlū-unshkotdāashang dalung lth shudang.

52. Dalung king-gogung-lēgas, dalung un gin ga lthwaugīgāashang! gin un tl ünshitlthing-as kikostlāwe tla sta dalung isdagun altha; dalung $\overline{\mathrm{u}}$ gum ai isdsa-ang-gun, waigien ai ga isdsa-ungkasasgai ishin ā dalung kẹigìdun, waigien hin il shouon.

53. Waigien tlistleuon sta il kägwaulandlu tukālung-lëalung isgien, Pharisee haade ishin la hanltha èlth-euon-īdan gien, gin kwon edē la tl ging-güshouon;

54. Waigien gin dāung-a il shudie keu tl edang-an.

IIA. 


\section{Chapter 12.}

WAUDLU tl shkūl-euon gudā ijan gien, gūt ing-gūt-hun tl isīdandlu, hin gyang: disciplesgai il shuda-tlagang-idan, Pharisee haade gia tlakulthdāwe sta lth ga gūtgung-ñ, gudung-ai stung is altha.

2. Waigien gin salgas wautliwon kang-gelthshang; gin kwulgun-gas wautliwon ishin un ūnshidēlthshang.

3. Althji alth tliku hun algwau dalung shūs otgasdlu tl gudung-ashang; waigien nahgwau àgwī gin tl geu-i dalung shūd-kwōlth-das nas ing-gu unsta tl shu-ashang.

4. Waigien hin dalung lth shudang, di tōalung isis, 'Tl lthū tl tldas gien, waudlā gum tliku tl waulthing-e kang-gans ga lth gum lthwaugang-ü.

5. Nung ga dalung lthwangalthing-as tlou dalung ga lth shudāashang; tla nung tildas dlā hetgwaugai ai tl dang-gai un nung dugwīas ga lth lthwaugū; āng, althunis ga dalung lthwaugie lang lth shugung.

6. Gum gu kwūtsagū tlēlth farthing gūstung-āgung-ung-ūsh? waigien gum swonshungga-hun Shanung-itlagadas kēshgīdung-unggung.

7. Waigien dalung kuts ou wautliwon kwaiindiāgung. Gum lth lthwaugang-u ; kwūtsagū kwon telg dalung kwōyāgung. 
8. Waigien hin dalung ga lth shugung, tl haada hang-gu di unsta nung shusdlu, Shanung-itlagadas gia angels-gai hang-gu la unsta ishin nung haada Git shu-ashang;

9. Tl haada hang-gu di da nung kudasdlu tlou, Shanung-itlagadas gia angels-gai hang-gu la da ishin lth kudāashang.

10. Waigien nung haada Git hanltha nung gushusdlu, la kalthshint tl gudung-ashang; Hants Las hanltha gushou dāung-a nung shudasdlu tlou, gum la kalthshint tl gudung-ungashang.

11. Waigien tlistleuon synagogue-nagai un ga ītlagadasgai isgien, ga îtlagadasgai ishin, ga dugwigasgai bang-gu ishin, dulung tl isdasdlu, tliku hang-gang dalung shūases ai gum gudung-ung tl lthkwīdadang-ūga ;

12. Tliku dalung shougai las nung Hants Las waudlu élthsdlu dalung shkotadäases altha, hin Jesus shouon.

13. Nung İtlagadas, gin talung dā-ungkashagun tīch lth di kwai di ga isdadi, waigien hin ga shkūlasgai swon il shudaian.

14. Nung îlthing-as, gīshdu dalung un di ging-kilisalang-lēgadas gien, gut āada ishin gin di tladīgälēgadaiang? waigien hin la il shudaian.

15. Gulth-gūt-gung-ū, waigien gin ga staltha wautliwon sta lth duman agung keng- $\bar{u}$; nung haada hēnung-gai gin kwon il dāas alth 
gum dugwīans altha, waigien hin tla il shudaian.

16. Waigien tla ga il këgang-an; Nung ginlthing-a gia tligai gu gin kwon inastlaian ;

17. Waigien odō il kwūlthalan gien, hin il shouon, Gushinū lth edāsīnī, gum tlījan gin inastlas ung-a lth isdalthing-e kang-gans altha?

18. Hin $\overline{\mathrm{u}}$ lth wau-ashang; corn-nagai ai ung-a lth tlinanung-ashang, waigien wau telg ga euonda Ith tloulthäashang; waigien gai ā corn-gai wautliwon isgien ginagai wautliwon ishin ung-a lth isdāashang.

19. Waigien hin althandang lth shudāashang, Althandai, tuda kwon dlu gin dung un kwon-gung; gudung-ung 1th wau shatusdi, Ithdanū ang-a lth tlika gwīlā, hin il shouon.

20. Dung kōnuns, algwau un dung althandai dung sta isdiāashang; waigien gīshdū gudā gin dung isdagun dāashang? waigien Shanung-itlagadas hin il shudaian.

21. $\bar{A}-\bar{u}$ tliku nung haada ēdung, gin kwōyā kenung un gūdā il isdas gien, gum Shanungitlagadas un il ginlthing-ansdlu-a, hin Jesus shouon.

22. Waigien hin gyang disciplesgai il shudaian, Althji alth hin dalung ga lth shugung, Gum lth hēnung-gang ai gudung-ung lthkwidadang-u, gin dalung tāases-a; gum lth lthüung ai ishin gudung-ung lthkwidadang- $\bar{n}$, gin dalung giandãases-a. 
23. Tl hēnung-gai torı telg ets gien, tl lthī ishin gin giandalthing-e telg ets altha.

24. Yelth odō lth kwūlthilthü; gum gin tldsans gien, ishin gum gin tlïidung-ung-gung ; gum tou-nai dāans gien, ishin gum corn-nai dāang-gung; waigien Shanung-itlagadas in lthdanūdagung-gung; hetît telg āgwī $\bar{u}$ dalung kwōyāgung!

25. Waigien gīshdū cubit swonshung dsīung dsing-illthdalthing-āgung, gudung-ung il lthkwidadas-kianan-a?

26. Gum gin dsūdsū-hun dalung isdalthingansdlu, gushintlou gin āda ai gudung-ung dalung lthkwidadagung-gung?

27. Stlukèstā odō lth kwulthilthū, tlikn inastlagung-us-ī-a ; gum lthong-gwilans gien, ishin gum gin lthgilgung-ung-gung; waigien hin dalung ga lth shugung, Solomon un yāgn̄dung-ägandlu, gum ashgai swon ging-an gin il gianda-tljū-ang-gang-an.

28. Kwulthkotajai $\bar{a}$ kun is $\overline{\mathrm{u}}$ aiat kougung, waigien adalth tsanusi tl isdāashang; waikian Shanung-itlagadas gin giandadasdlu, gum gu wau telg gin dalung il giandadang-ashā-ash, lthing-an dalung yetdas-a?

29. Waigien gin dalung tãases isgien, gin dalung nīlthises ishin lth gum da dî-ing-ung-î̀, ishin lth gum gudung-ung kwondang-ū.

30. A gin isis wautliwon da gwaigang hētligai gu isis da dí-ing-gung-sĩ altha; waigien 
a gin isis ga dalung stalthas un $\overline{\mathrm{u}}$ dalung Aung unshidung.

31. Waigien lth kingdomgai da lang-a dìing-ū, waigien a gin isis dalung gia isīlthishang.

32. Lumado gūi-knutsū dalung isis, gum lith lthwaugang-ū ; kingdomgai dalung ga isdie un dalung Aung gudung-ai las altha.

33. Gin dalung dāas alth giadahū-ū, waigien alth tla da tlāadū, dāla gwau-alē gum kēgēlthang-ases lth kenung un tloulthū, gin kwōyā gum tlan ellthang-ases lth shi ga isd $\bar{u}$, gaigu gum kwolthdale katlans gien, ishin gum stluknm gin tl-dāung-ëdung-ung-gung.

34. Gyāgu gin kwōyā dalang-a isis gu dalung: gudung-ai ishin isises altha.

35. Ung dalung dl-juts gien, kāsōdou-e ishin dalang-a ōgai lāgung;

36. Waigien tl îtlagīda gūinēl lagunung-ai sta silthgang stēlgai da tl haada khatsū-gungus ging-an lth etdu; tlistleuon il katlas gien, keu-e il shkustagasdlu, hawīdan la keu sta wau sta tl da-ostlie una.

37. Tlistleuon nung itlagadas katlas gien, il gītsādalung khatsūs il kensdlu, yenki tla un lā-gung-gung: yenkien dalung ga lth shugung, ung il dlj-gūsdlā-ashlang, waigien tl lthdanūe un tla il kil-tlâ- $\bar{u}$-ashang, waigien il katlas gien tla alth il dai-ing-ashang.

38. Waigien shkiana-stang ai il katlas gien, shkiana-lthōnulth ai ishin il katlas gien, hi 
tl ets il kensdlu, a ga gitsadasgai un yenki lāashang.

39. Waigien 1th adshi un ūnshidū, ashkoshgut gūt nung kwolthdalēga katla-ung-kasas un nung nuh-légas ūnshidasdlu, il shkianasgaia-hung-gung, waigien gum nai lang-a tl tla-helthilè keu il tutīdlishga-ung-ahung-gung. 40. Dalung ishin lth ung elthgīdū; giadlu gum il katla-ung-ashang dalung gudunsdlu, nung haada Git katlā-ases altha, hin Jesus shouon.

41. Shalana, itil un swonan gu adshi kēgangai dung shudi, tl wautliwon-hun un gwau? waigien hin Peter shouon.

42. Waigien hin Shalana shouon, Gīshdū nuh duman kang-lē lā isgien ishin kutung-a ijung, il îtlagīda gyang nai duman il kendas gien, time-gai wandlu èlthsdlu tla ga il gĩdie una?

43. Il itlagīda katlas gien, hin il ets lang-a il kensdlu, yenki wunung gìtsadas un lāashang.

44. Yenki dalung ga lth shugung, gin il dāas wautliwon duman la il keng-ilthdāashang.

45. Di itlagīa katlagai jing-ā-gung, hin wunung gitsadas gudunsdlu tlon, waigien ga gītsada îlthansidasgai isgien, ga gitsada jāadasgai ishin il shkidung-īdsdlu, waigien il lthdanū-ids gien ishin il lum-gasdlu,

46. Wunung gitsadas gum khatsui-uns gien, ishin gum time-gai un il ūnshidang-gundān, il itlagida katlāashang, waigien la il shkìdsulth- 
euon-shang, waigien gum ga lāansgai shu-wī la il isdāashang.

47. Waigien wunung gitsadas itlagidang gudung-ai un ūnshits gien, waigien gum ung il ëlthgi-dans gien, ishin gum il gudung-ai ging-an il edansdlu, la tl shkidung-euonshang;

48. Waigien gum un il unshidans gien, gin agunan la tl shkidung-ai las il isdasdlu, lthing-an la tl shkidung-ashang. Waigien nung-ga-hun gin kwon isdiasdlu, la sta gin kwon ga tl stalthāashang; waigien nung-gahun gin tl isda-kwonsdlu, wan telg la alth gin tl ginung-ashang.

49. Hêt-tligai ing-gu das lth kātie un lth katlang; waigien wèt hun ōgulthsîdlu di gudung-ai lādādung-gung!

50. Baptism alth di tl baptize-die lagung; waigien ëlthgīgai keu di gudung-ai lthkwīdewon-gung!

51. Hêt-tligai gu gudang dlaia lth isdie mn lth katlang gu dalung gudung-ūsh ? Gãanū, gut hanltha tla lth tla-elgai un lth katlang dalung ga lth shugung;

52. Adshi sta nuh tî-swonshung à tl tlēlth naansdlu, gūt ãada agung tl tlāadses altha, tl lthonulth tl stung hanltha ets gien, tl stung ishin tl lthonulth hanltha etishang.

53. Gūt āada agung tl tlāadshang, nung aung-as git-ang hanltba ets gien, nung git-as ishin aung-ung hanltha etishang; nung oul-as 
gī-jang-ung hanltha ets gien, nung gūjas ishin oul-ung hanltha etishang; nung jūnas git-ang jā hanltha ets gien, il git jā ishin jūn-ung hanltha etishang, hin Jesus shouon.

54. Waigien tl shkī'as hunisun hin il shudaian, Tlistleuon jâ. gwau yan shi ga is dalung: kensdlu, Haskädalgung, hawìdan hin dalung shugung-gung; waigien wai ging-an ijunggung.

55. Waigien tlistleuon ousik tudsūs dalung: kensdlu, Tukhousda-ung-kashang dalung shugung-gung; waigien wai ging-an ijung-gung.

56. Dalung gudung-ai stuns, hêt-tligai hung isgien, sha-tligai hung ishin tliku ets un dalung inshidung-gung; gushintlou gum adshi timegai tliku ets un dalung unshidang-gung?

57. Gushintlou dalung kodan gum gin lā un innshidung-ung-gung?

58. Dung hanltha nung ets alth nung gingkilisalang-lēgas à dung kasdlu, hawun dalung istalgundān gin agunan dung hanltha il ets tlan êlthdie dung isdagudung-enon-ai lāgung; gum ging-an dung edans gien, nung gingkilisalang-lègas à dung il isdàashang, waigien nung ging-kilisalang-lēgas nung kēchitlēgas ga dung isdāashang, waigien nung kēchitlēgas kēchitnai ai dung isdāashang.

59. Gin wantliwon dung shougai keu gum tliku sta dung kāgwaulthing-e kang-gang-ung lung ga lth shugung, hin Jesus tl shudaian. 


\section{Chapter 13.}

GALILEE haade hawun gin tīiga dlāasagundān, gin tīigagai ai alth Pilate tl ai gu shu-wìt tla-ēlan waudlu gu tl ijan la ga alth gialthandai-an.

2. Waigien hin hanglthang tla il shudaian, Galilee haade a gin isis andang-an alth gu Galilee haade wautliwon telg dāung-aiang dalung gudung-ūsh ?

3. Gãanū; gum gudung-ung dalung stīthdansdlu, wai ging-an dalung wautliwon gīashang dalung ga lth shugung.

4. Siloam gu nuh jing ga tlāalth waugu stanshang-a giwī whōndsaian gien kōtulthgaian gu Jerusalem gu tl nāans wautliwon telg tl dāung-ai-ang dalung gudung-īish ?

5. Gāanū; gum gudung-ung dalung stīlthdans-dlu, wai ging-an dalung wautliwon ginashang dalung ga lth shugung, hin tla il shudaian.

6. Waigien adshi kēgang-ai alth il kēgangan; Nung īlthing-a gia vine-lthkai kwulthkotajai ā fig lthkai lthkajāwon; waigien ān gut kougai il keng-atlagan gien, da il dì-inchgaian.

7. Gwokeng, tuda lthōnulth gut adshi fig. lthkaigai gut ān kougai da lth keng-tlagunggung, waigien da lth di-inch-gagung-gung ; la lth shkika; gushintlou adshi kōie ishin 
ging-gang-an gougä-gung? waigien hin vine lthkai duman kang-lē il shudaian.

8. Waigien hin hanglthang la il shudaian, Nung itlagadas, kwai lth housen kenèt swonshung wau gu isda, waigien la odōsi lth ltbais gien, Ith chinhädāashang;

9. Waigien waudla ān la gut inastlas gien, läashang; gum la gut inastlansdlu tlou, la dung shkikāashang, hin hanglthang il shouon.

10. Waigien sabbath shantlanai ai synagogue-nagai tī-swon ā gin tla il shkotadaian.

11. Waigien nung jada tuda tläalth waugu stan-shang-a hants alth stigan gu ijan; waigien il iskup-jäwon gien, gum tliku agung il yädalthing-e kang-gang-an.

12. Waigien tlistleuon Jesus il kang-andlu, la un il ai-ang-an, Nung jadas, stigai sta ung-a dung kogun-gung, waigien hin la il shudaian.

13. Waigien la ing-gu stlang il isdaian, waigien hawidan il yägalan, waigien Shanungitlagadas ä il kil-lägan.

14. Waigien Jesus sabbath shantlanai ai nung tl-ligalan alth synagogue-nagai un nung: itlagadas kalthi-yilthdaian, waigien hin ga shkūlasgai il shudaian, Tl haada gut lthonggwilie un shin tlū-unilıh ijung; althji alth althsgai shantlanai gut dalung ligulai un adlun dalung istlagai lagung, sabbath shantlanai tlou gum un lāung-gung, hin tla il shudaian. 
15. Waigien Shalana hin hanglthang il shudaian, Dalung gudung-ai stuns, gum gu dalung wautliwon hun sabbath shantlanai ai mishmush isgien, giūdan ishin gyagu keu-giāang-as sta ai ung-a dalung tlā-ads gien, gyagu hūtlāases à dalung al-istalgung-ung-ūsh ?

16. Waigien gum gu anung jadas, Abraham güjang-a isis, Satan tuda tlāalth waugu stanshang-a këchguwaudaian, sabbath shantlanai ai ai otadai lāung-ush? hin il shouon.

17. Waigien hin il shūgundān, la hanltha gd edan-gai wautliwon èdastlaian; waigien gin unyãgudung-a wautliwon il isdaian alth ga shkīlasgai wautliwon tlika gwīlāgan.

18. Waigien hin il shouon, Gīsh ging-an $\bar{u}$ Shanung-itlagadas gia kingdomgai ëdung? waigien gīshgū lth kha-äashang?

19. Mustard malthe skā-swonshung nung haada isdaian, tōkanai ã ung-a il tldsaian gingan $\bar{u}$ edung; waigien inastlaian gien, gin lthkai isēlan; waigien siet hetit isis tlats ing-gut naang-gang-an, hin il shouon.

20. Waigien hin housen il shouon, Güshgin Shanung-itlagadas gia kingdomgai lth khaàashang?

21. Nung jada tlukulthdou isdaian gien, shibli kwīda lthōnulth à il salan gien, ì tlukulth-ōdsūe keu wau ã ijan ging-an $\bar{u}$ èdung, hin il shouon.

22. Waigien lana isgien, lana kntila ishin 
kalthgut il kāgan gien, gin tla il shkotadagangan, waigien Jerusalem gwī ga il kāgang-an.

23. Shalana, ga kogunsgai gu gū-kutsū? waigien hin la nung shudaian. Waigien hin tla il shudaian,

24. Keu tumdala ai isdsie lth isdagudungeuon-ū; tl kwon $\bar{u}$ ai isdsie da guduns gien, wau odō eshgāashang dalung ga lth shus altha.

25. Tlistleuon nung nuh-lēgas giāas gien, keu-e wau ung-a il da-ashgutgīsdlu, kyāgūst dalung tljūgiang-īds gien, keu-e dalıng shkwīdagas gien, Shalana, itil keu sta lth wau sta da-astli, hin dalung shū-ash-ang; Gum dalung un di ūnshidans gien, tligist dalung is hunisuu gum un di ūnshidang gung, waigien hin hangIthang dalung il shudāashang;

26. Dung hang-gu talung lthdanūgun, waigien keu kitadias a ishin gin itil dung shkotadagini, hin itan dalung shū-ìdshang;

27. Gum dalung un di unshidang-gung, tlijī sta dalung isis ishin gum un di unshidang-ung dalung ga lth shugung; di sta lth issū, gin dāung-a isdalēalung dalung isis wautliwon-a, waigien hin il shuashang.

28. Gaigu tl saigas gien ishin tsing-ung tl kōkou-nanung-ashang, thistleuon Abraham isgien, Isaac ishin, Jacob ishin, ga prophetgasgai wautliwon ishin, Shanung-itlagadas gia lingdomgai gu isis gien, dalung tl kyā ga dang-gas dalung kensdlu-a. 
29. Waigien hēōgūst isgien, jāgūst ishin, kāāūsta ishin, ousik yā sta ishin, tl istlas gien, Shanung-itlagadas gia kingdomgai gu tl tlōwong-ashang.

30. Waigien òtgwau ăgwī ga isisgai îlthi kwūniāashang, waigien ga kwūniasgai ìlthi ishin ōtgwau āgwī isisang, waigien hin tla il shudaian.

31. Waudlu Pharisee haade ilthi istlagan, Kägwalth ang-a lth kaid, Herod dung tīie da guduns altha, waigien hin la tl shudaian.

32. Waigien hin tla il shudaian, Istīidū ang-a lth hin nukudsigai shudu, Aiat isgien adalth ishin hants dāung-as kyā ga lih dangwos gien, ishin tla lth tla-ngistlä-ashang, waigien shin lthonulthsdlu di elthgīishang,

33. Waikian aiat isgien, adalth ishin, adalth dāalīst ishin keu-e gut ung-á lth kogai lāgung; gum tliku Jerusalem kyägust nung prophetga goulthing-e kang-gans altha.

34. Jerusalem, Jerusalem, ga prophetgasgai dung tldas gien, dung ā ga kil-itsgas-gai ishin kwau alth dung kunlthūgung-gung! gīshīdlu shkou jada gitalung hyang hetgwī gudā isdaguns ging-an dalung gitalung guda lth isdalthing-āgun, waigien ga dalung gwou-gun !

35. Dalung gia nai hìlūgung; Shalana kyē $\bar{a}$ nung katlas $\bar{u}$ lāgung, hin dalung shougai keu, gum di dalung keng-ung-ashang dalung ga lth shugung, waigien hin tla il shudaian. 


\section{Chapter 14.}

WAIGIEN tlistleuon sabbath shantlanai ai Pharisee haade un ga itlagadasgai swon gia nai ai lthdanou un il kudsaiandlu, la à tl lthotsagang-an.

2. Waigien sti dunlthia alth nung stigan la hang-gu ijan.

3. Waigien tuking-gogung-lēalung isgien Pharisee haade $\bar{a}$ ishin Jesus gushouon, Sabbath shantlanai ai nung tl tla-ngīstlie gu lā? waigien hin il shouon.

4. Waigien gum tl kìlang-an. Waigien la il isdaian gien la il tla-ngīstlaian, waigien la il kaidaian.

5. Dalung swon gia geudanai isgien, mishmüsh-gai ishin, untl hēla ai dlūīdsasdlu, gum gu hawīdan sabbath shantlanai ai sta la il dung-dlistang-ash? waigien hin tla il shudaian.

6. Waigien gum tliku ashgai hang-ga tl shoulthing-e kang-gang-an.

7. Waigien kēgung alth gia un tl ai-ang-an ga il kegang-an, tlistleuon tudsgwaugai aulthgahung-we tl isdas il kang-andlu-a; waigien hin tla il shudaian,

8. Tlistleuon gū ineël lagunung-ai un dung un nung ai-insdlu, gum tudsgwaugai aulthgahung-we ing-gu tl kou-ang-ga; ging-an dung; 
etsdlu, dung telg nung unyāgudung-a un il ai-ins gien,

9. Waigien dalung un nung ai-ang-an katlas gien, Unis lth adlun kouda, hin dung il shudalthing-ā-gung; waigien èda dung-alth lth kyāgwau āgwī dung kou-ashang.

10. Waigien tlistleuon dung un tl ai-insdlu lthkyāgwau āgwī tl kouga; tlistleuon dung un nung ai-ins katlasdlu, Di tō-wī, tudsgwau lth kou-i, hin dung il shudie una; wandlu dung alth ga lthdanūgaai wautliwon hang-gu dung uny āgudung-āashang.

11. Sha ung nung edasdlu, hēt ga il èlthses altha; waigien hēt ung nung edasdlu, shī ga il èlthshang, hin tla il shudaian.

12. Waigien la un nung ai-ang-an ishin hin il shudaian, Tlistletzon lthdanou un tla un dung ai-insdlu, tō-alung isgien, kwai-alung ishin, tō-alung yikĩ ishin, dung odō ga itlagada nāans ishin gum un tl ai-ing-ung-ga; ging-an dung etsdlu, gai ishin dung un ai-ing-ashang, waigien dung ga giashougèlthshang.

13. Waigien tlistleuon tla un dung ai-insdlu, ga kong-ushgitaigasgai isgien, ga tlikiasgai ishin, ga kwolū kōdasgai ishin, ga hangkagısgai ishin un tl tl ai-ing-ga.

14. Waigien dung un lāashang; gum dung ga gin alth giashoulthing-e kang-gans altha ; ga lasgai kotal sta kalthougadunsdlu, dung ga giashou-gãases altha, waigien hin la il shudaian. 
15. Waigien tlistleuon la alth ga lthdanouon-gai swon adshi gudang-andlu, Shanungitlagadas gia kingdomgai gu nung lthdanūsdlu, yenkien la un lāashang, hin la il shudaian.

16. Waigien hin la il shudaian, Awulth nung haada tou kwon tling-alang-an gien, tl kwon un il ai-ang-an;

17. Waigien lthdanue waudlu êlandlu, gìtsadang il kil-kaidan, Ūtlāū, gin wautliwon èlthgìs altha, hin giā un il ai-ang-an il shudie una.

18. Waigien tl wautliwon gut ging-an ga gwaugalan. Kwulthkotis lth dahgun, waigieu lth keng-āgai lāgung; gum à kogai gwi di edang-gung, hin nung swon il shudaian.

19. Mishmūsh lthong-gwila-aia tlāalth 1 th dahūgun, waigien la lth isdagudung-a-ungkashawong; gum à kogai gwī di edang-gung, waigien hin ishin nung swon shouon.

20. Lth jadanēlgun, althji alth gum ā lth kalthing-ang-gung, waigien hin ishin nung swon shouon.

21. Waigien nung gītsadāgan katlagan gien; adshi itlagidang ga il shudaian. Waudlu nung nuh-lēgas kalthi-yilthdaian, Ilnagai keu-e kitadias a lth ga kong-ushgi-taigasgai isgien, ga tligiasgai ishin, ga hangkagasgai ishin, ga kwolū kōdasgai ishin althgwī isdi, waigien hin gītsādang il shudaian. 
22. Nung itlagadas, tliku di dung shudagun ging-an lth waugun, waigien hawun tālägung, waigien hin nung gitsadas shouon.

23. Waigien nung itlagadas hin gītsädang shudaian, Keu ālthas isgien, kwulthkotajai a ishin lth kagwulth, waigien anä ga tl isdie lth isdagudung-euon-ung, nai dinna stahgai una.

24. Gia un lth lthaigun-gai swon-hun gum lthing-an giagun tou-e tâ-ung-ashang dalung ga lth shūs altha, hin il shouon.

25. Waigien tl shkūl-euon la alth ijan; waigien tla gwī il hung-âwon gien, hin tla il shudaian,

26. Dì un nung katlas gien, gum aungung isgien ou-ung ishin, jā-ung ishin, gitalung: ishin, kwai-alung ishin, jas-alung ishin telg il keng-uns gien, gum hēnung-gang telg ishin il keng-unsdlu, gum tliku giagun disciplegai il islthing-e kang-gang-gung.

27. Gum gyang shkau-amagai nung isdans gien, gum di dlā ishin il kā-unsdlu, gum tliku giagun disciplegai il islthing-e kang-gang-gung.

28. Dalung swon nuh jing tloulthie da gudunsdlu, gum gu il kou-tlagans gien, tlistleu ai gin hīlūases il kwai-indagung-ung-ūsh, waudlu dālē lang-a etgai un il ūnshidai una?

29. Gum ging-an il wau-unsdlu, keusi il tloulthas gien, elthgīdie à il dsigiasdlu, ga kensgai wautliwon la alth kilth-nang-īds gien, 30. Anung ilthing-as nuh tloulthiēedun, wai- 
gien èlthgìdie à il dsigīigun, hin tl shugunggung.

31. Waigien nung king-ga nung king-ga kulat un ailthda-ungkasasdlu, gum gu il kous gien, soldier haade thousand tlāalth alth ga thousand tlāalē stung dung-alth la un nung katlas un ailthdie un il dugwīgai odō il kwūlthilth-tlagang-gung-ung-īsh ?

32. Waigien gum un il dugwiansdlu, hawun la un nung ailthda-ung-kasas jīng-āgundān, la $\bar{a}$ gushou il hundsũdas gien, gum la un il ailthda-ung-ai sing tlistleu gin ga il stalthie da la alth il kiānung-gung-gung.

33. Althji alth dalung swon gin il dāas wautliwon ga gum gwaugulthansdlu, gum tliku giagun disciplegai il islthing-e kang-ganggung.

34. Tung ù gin lā ijung; tung-ai tung-hōgie guīlthsdlu tlou, guish alth $\bar{u}$ gin tl tung-adāashang?

35. Tligai un isgien, chin-hā un ishin gum lāung-gung; hing-an tl dang-gung-gung. Gin gudung-ai un nung geu-wos lth akwon gin il gudunda, hin tla il shudaian.

\section{Chapter 15.}

W AIGIEN giashouganung-lēalung isgien ga dāung-asgai wautliwon ishin il kil gudung-ai un aānan la un istlagan. 
2. Waigien Pharisee haade isgien, tukālunglēalung ishin guâung-ai stigan, Anung îlthingas ga dāung-asgai isdas gien, tla alth il lthdanūgung-gung, waigien hin tl shouon.

3. Waigien adshi kēgang-ai alth tla ga il kēgang-an,

4. Dalung swon lumadō hundredgai dāas gien, swon il gūdasdlu, gum gu tlāale tlāalthswonshung-ḡi waugu tlāalth-swonshung-gū tligunltha il inas gien, nung gāwon il kēie keu da il dì-ing-gung-ung-ūsh ?

5. Waigien tlistleuon la il kēas gien, shkeuung gwī la il isdas-dlu, tlika il gwwūlāgunggung.

6. Waigien tlistleuon il katlasdlu, tō-alung isgien, la kwulth-it ga nāansgai un ishin il ai-ins gien, Di alth lth tlika gwūlāū, lumadō dina gūgun lth kēigun altha, hin tla il shudagung-gung.

7. Tl haada lā tlāale tlāalth-swonshung-gū waugu tlāalth-swonshung-gū gum gudung-ung stīlthdie ga stalthans telg, nung dāung-a swonshung gudung-ung stīlthdasdlu, sha-tligai gu tlika tl gwūlang dalung ga lth shugung.

8. Waigien nung jada dāla kē-tlāalth daas gien, kē-swon ung-a il gūdasdlu, gum gu kāsōdou il ōgulthdas gien, nai kālthi il lthkyoudals gien, ung-a il kēie keu, da il dī-ing-euongung-ung-ūsh?

9. Waigien thistleuon il kē-asdlu, tō-alung 
isgien, la kwulth-it ga nāansgai ishin un il aiins gien, Di alth lth tlika gwūlāū, dāla lth gudagun ung-a lth kēigun altha, waigien hin il shugung-gung.

10. Wai ging-an nung dāung-a swonshung gudung-ung stilthdasdlu, Shanung-itlagadas gia angel-gai hang-gu tlika gwülā ising dalung: ga lth shugung, hin Jesus shouon.

11. Waigien hin ishin il shouon, Nung îlthing-a gittalung stang-an ;

12. Aung-a, gin dung dāas tīch lth dāungkashagun lth wêt di ga isdi, waigien hin nung dōnas aung-ung shudaian. Waigien gin il dägan gut ãada la ga il tlāadāwon.

13. Waigien gäwun èt-lang-gun-dān nung dōnas gin wautliwon gudä isdaian, waigien tliga jīng-a $\bar{a}$ il kaidan; waigien gaigu gin dãung-a il isdas ai gin il dāgan ung-a il tlahīlāwon.

14. Waigien tlistleuon ung-a il tla-hîlūōdsou-ondlu, gai tligai gu yenki tou hīlāwon; waigien il kong-ushgitaigèlan.

15. Waigien il kaidan gien, althsgai tligai haade swon ga il dlishgadan; waigien gwoshou il lthdanūdie un kwulth-kotajai à ung-a la il kil-kaidan.

16. Waigien gwoshou gin kul tas tugai da il gu-dang-an; waigien gum nung swonshunghun gin la ga isdang-gang-an.

17. Waigien tlistleuon aigang agung il gî- 
kēidandlu, hin il shouon, Di aung gitsadalung kwon tou tas gien, wausta ishin kāwīdung-gung, waigien adlun kwit alth di gūgung!

18. Lth kālthūs gien, aung-ung a lth kaidshang, waigien hin la lth shudāashang, Aung-a sha-si un di dāung-gun, waigien dung hanggu ishin ;

19. Gum housen, Di git, hin di dung kyādie un di lā-ung-gung; gìtsadalung swon ging-an Ith di elthdi, hin la lth shudāashang, hin il shouon.

20. Waigien il giāagan gien, aung-ung un il katlagan. Waigien hawun il jīng-āgundan il aung il kang-an, waigien la kalthshint il gwaulan, waigien il odan gien, il hel ga agung il shkwōdlishgadan gien, la il shkwūntlaian.

21. Waigien hin il git il shudaian, Aung-a, shasi un di dāung-gun, waigien dung hang-gu ishin; waigien gum housen, Di git, hin di dung kyādie un di lāung-gung, hin il git il shudaian.

22. Waigien il aung hin gitsadalung shudaian, Hawīdan lth althgwī kodats lādsilthīs isdū, waigien lth ai il tla-kudsū; waigien lth stlīilthū il stlai ai isdū, waigien lth stushgaga ai ishin il tl-tulthū ;

23. Waigien lth mishmūsh giti tla-ai-istlias althgwi isd̄i ang-a tîi-ū; waigien talung tas yien, tlika talung gwūladsun;

24. Di git isis kotulgun housen hēnanstlas 
altha; il gūgun, waigien la talung kēiang, hin il shouon. Waigien tlika tl gwūlā-ēdan.

25. Waigien il git kwai-asi kwulth-kotajai à ỉjan; waigien il kāgundān nai un il dōngèlandlu, gin tl tla-king-uns gien tl hyalths il gudang-an.

26. Waigien ga gitsadasgai swon un il aiang-an gien, tliku adshi etgai da la alth il kianang-an.

27. Dung dōn $\bar{u}$ katlang, waigien dung aung mish-mūsh gîti tla-ai-istlias tiigun, silthgang duman la un il koguntlas altha, waigien hin la il shudaian.

28. Waigien il kalthi-yilthdaian gien, kudsie ga il gwou-aian; waigien il aung katla-waugan gien, la un il lthai-idsaian.

29. Waigien hin hanglthang aung-ung il shudaian, Gwokeng, tuda kwon gut dung un lth lthong-gwilgun, waigien gum dung gia king-gogung-ai ilā lth edang-gun; waigien tō-alung alth tlika lth gwūlagai un gum kimdai gîti swonshung-hun di ga dung isdanggun ;

30. Waigien tlistleuon a dung git is, gin dung dāas jā-ung-lēalung ai tla-hīlāwon, katlagandlu, mishmüsh giti tla-ai-istlias la un dung tii-aian, hin il shouon.

31. Lthken, di kwulth dung isgigung, waigien gin lth dāas wautliwon dung gia ijung.

32. Tlika talung gwīlas gien, ishin itil gu- 
dung-ai ligai ū lāgung; a dung dōn is kotulgun gien, housen il hênanstlas altha; waigien il gügun, waigien la talung kēiang, waigien hin la il shudaian.

\section{Chapter 16.}

WAIGIEN ga disciplegasgai ishin hin il shudaian, Nung itlagada duman gin kang-lēga dãagan; althunis $\overline{\mathrm{u}}$ gin $i l$ daas ging-gang-an lang-a tla-hīlūgung-ung tl haada la ga shou-on.

2. Waigien la un il ai-ang-an, Gūsh dung isdaian $\bar{u}$ tl shudas lth gudung-gung ? lthonggwilie tliku etsi wautliwon lth ung-a di ga shudi; gin duman dung kens tlan dung elthdie las altha, waigien hin la il shudaian.

3. Waigien duman gin nung kang-lēgas hin gudang-an, Gushinu lth etishang, tlan di îtlagìda di lthong-gwildas altha? gin Ithaigai un gum di dugwīāng-gung; gin ginung-ai à ishin di èdang.

4. Tlistleuon tlan di il lthong-gwildasdlu, gyang nai $\bar{a}$ di tl isdie un tliku lth etses un di inshidung, hin il gudang-an.

5. Waigien il itlagida alth ga gia-shildiāgan swōnā-hun il ai-ang-an gien, Gishìdlu di îtlagida alth dung gia-shildiägung? Hin nung swon il shudaian.

6. Kwīdou hundredgai swonshung ā tō lth 
shildiāgun, waigien hin il shouon. Kōgīnē lth ung-a isda, waigien lth kou ang-a tläale tlēlth kālung, waigien hin la il shudaian.

7. Gisshīdlu da ishin gia-shildiägung? waigien hin nung kulat ishin il shudaian. Kwidou hundredgai swonshung $\bar{a}$ wheat lth shildiāgun, waigien hin il shou-on. Kōginē lth ung-a isda ang-a tlāale stanshang-a kālung, hin la il shudaian.

8. Waigien duman gin nung kang-lëgas kutung-ā-gan il itlagỉda gwūlägan ; otgagai gitalung telg adshi hēt-tligai gitalung duman ung kang-gai un kutung-as altha

9. Waigien hin dalung ga lth shugung, Ginlthing-a dāung-a alth tō-alung kenung un Ith tloulthū; tlistleuon hīlūsdlu, giang-u-nai et swonuns ai dalung tl isdie una.

10. Gin dsūdsū duman nung kang-gai lasdlu, gin kwon ishin duman il kang-gai lāgung, waigien gin dsūdsū gum. duman nung kanggai läunsdlu, gin kwon ishin gum duman il kang-gai lā-ung-gung.

11. Althji alth ginlthing-a dāung-as gum duman dalung kang-gai lāunsdlu, gīshdù ginlthing-a yiki isis duman dalung kendāashang?

12. Waigien gum tl kulat gia duman dalung: kang-gai lāunsdlu, gīshdū dalung ga gin dalang-a isdāashang?

13. Nung gitsada gum tliku tl itlagada stung un lthong-gwilalthing-e kang-gang- 
gung; nung swon telg il kens gien, nung swon il kwōyā-dãases altha; gum ging-an il edansdlu, nung swon il gwũlas gien nung swon ga ishin il gwou-wau-ashang. Shanungitlagadas un isgien, ginlthing-a un ishin gum tliku dalung lthong-gwilalthing-e kang-ganggung, hin Jesus shouon.

14. Waigien Pharisee haade dāla kwōyãdagang-an adshi wautliwon gudang-an; waigien la alth tl kilthnang-an.

15. Waigien hin tla il shudaian, Dadung $\overline{\mathrm{u}}$ tl haada hang-gu agung lädagung-gung; Shanung-itlagadas tlou dalung gudung-ai un ūnshidung; tl haada shu gin lā-ewon isis Shanung-itlagadas un dā-ung-euons altha.

16. John katlagai keu king-gogung-ai isgien, ga prophetgasgai ishin ijan; althsgai sta Shanung-itlagadas gia kingdomgai gialthing-e alth tla geu gn tl gūshūs gien, tl haada wautliwon ai lthkwid-hī-jandsagung-gung.

17. King-gogung-ai à gin ltholth kutsū knuswonshung isis ging-gang-an elgai telg, shatligai isgien hêt-tligai ishin hīln̄è yang-• alang.

18. Jā-ung nung dans gien, nung kulat il inēlthsdlu, hing-an la alth il tīgādang; waigien jā-ung nung dans gien, nung kulat il jā inēlthsdlu, hing-an la alth il tịgädang, hin Jesus gyang disciples-gai shudaian.

19. Waigien hin ishin Jesus shouon, Nung 
ilthing-a itlagadāgan, waigien gin ölthulth isgien, giang- $\bar{u}$ la ishin il giandagang-an, waigien tou lā shan-tlan wautliwon il tāgangan ;

20. Waigien nung kong-ushgitaiga, Lazarus bin kyāgan, keu-e à lang-a dlūdagang-an, waigien lè gin koutljāwon,

21. Waigien nung itlagadas gia table-gai sta kwô-kung-ou kūgas tugai da il gudunggang-an; waigien ha istlas gien, lè gin kous tang-gang-an.

22. Waigien gum et-lang-gundân nung kong-usbgi-taigas kotalan, waigien ga angelgasgai Abraham kun à il isdaian; nung itlagadas ishin kotalan waigien la tl lthēôwaian.

23. Waigien Hades à il waugang-andlu shi ga il këilthaian, waigien wochgwau āgwi Abraham isgien, Lazarus il kun a isis ishin, il kang-an.

24. Waigien il sailth-kiägang-an, Aung-a, Abraham, di kalthshint lth gudung, waigien Ith Lazarus di ā kil-is, undl ai stlukung-ung $\mathrm{kwūn}$ il isdas gien, di tong-il il tl-tutulthdie una; adshi ōsi à di wauguns altha, waigien hin il shouon.

25. Waigien Abraham hin shouon, Lthken, dung hēnung-ägan nūt dung chäagai-lāgan, svaigien Lazarus chäagüdang-gang-an * ai lth kēid; waigien wēt adlun il chãagai-lāgung, dung tlou waugung-gung. 
26. Waigien adshi wautliwon ilā, itil isgien dalung ishin äadsi tligai lthdingn-â-evon-gung, adshi sta dalung $\bar{a}$ itsgai da ga guduns gum wai ging-an isans gien, houshgai sta ishin gum itil à tl isang-ai una, hin il shouon.

27. Althji alth di aung gia nai $\bar{a}$ la dung kil-kaidai da dung alth lth kianung-gung;

28. Di dōnalung tlēthas altha; la $\bar{a}$ il gushu-wos gien, la ishin adshi tligai gu tl wauguns un gum istla-ung-awē una, hin il shouon.

29. Moses gia kōginē isgien, ga prophetgasgai gia kōginē ishin il dāawong; akwon lth tl kil il gudung-dū, waigien hin Abraham shoun.

30. Gāanū, aung-a Abraham, kotal sta la à. nung kaidwosdlu tlou, gudung-ung il stîlthdawau-ashang, waigien hin il shouon.

31. Moses kil isgien, ga prophetgasgai kil ishin gum il gudung-ung-wosdlu, kotal sta nung kalthūs-kianan gum il kil il yetdawaulthing-ang-gung, waigien hin la il shudaian, hin Jesus shouon.

\section{Chapter 17.}

WAIGIEN hin gyang disciplesgai il shudaian, Gin $\mathrm{tl}$ ging-dldulthdalthing-e gum tliku gū-êl-lthing-e kang-gang-gung; nung agunan isĩ un tlou gin ga lthwangigāashang! 
2. A ga ikutilasgai swon'nung ging-dldulIthdie telg, mūl a a kwau-e il heli sta tl keukēous gien, chān ga la tl kātagie $\bar{u}$ la un lagung.

3. Duman lth agung keng-gung-u; dung dōn gin dāung-a isdasdlu, la tl stidaga; waigien gudung-ung il stilthdasdlu, la kalthshint tl gudung-ga.

4. Waigien shantlan swonshung gut dung il tloulthdāung-a-dsikwausdlu, waigien dung gwì housen il stêl-dsikwaus gien, Alth di gudungai stigung, hin il shusdlu, la kalthshint dung gudung-ai lāgung, waigien hin gyang disciplesgai il shudaian.

5. Talung yetdie lth wauga agung kashgada, waigien hin ga apostlesgasgai Shalana shudaian.

6. Waigien hin Shalana shouon, Mustard malthē ska-swonshung dlu dalung yetdasdlu, Agung lth dunlth-kostad ang-a chān agung tldsada, hin a sycamine lthkai isis dalung shuda-lthing-àgung, waigien dalung kilth ging-an et-lthing-āgung.

7. Dalung swon gitsada tliga uldsulths gien, ishin lumadō duman il kensdlu, tlistleuon kwulthkotajai sta il katlasdlu, Hawidan lth kou ang-a lthdanū, hin gu la il shudalthing-a?

8. Tou lth di un tling-älung ang-a un dljgūs-tla, waigien lth Ith lthdanū-ēlthgigai keu duman di keng; waigien waudla dā ishin lthdanū-ashang, gum gu hin la il shudang-ash ? 
9. Nung gitsadas la kilth ging-an etsdlu gu, la à il kil-lāgung?

10. Wai ging-an dalung ishin, tlistleuon gin alth tuking-gogung-as wautliwon dalung elthgīdasdlu, Gītsada dāung-a ū itil ijung; gin talung isdie las swonan talung isdagun, hin tl shu-üga, waigien hin Shalana shouon.

11. Waigien hawun Jerusalem $\bar{a}$ il istalūgundān, Samaria tligai isgien, Galilee tligai kalthgūt ishin il kāgan.

12. Waigien lana ai il kudsang-kashagandlu, tl illthansida tläalth leprosy alth stigan la un istlagan, waigien wochgwau-hun la sta il tl-jügiang-āwon ;

13. Jesus, nung Itlagadas, itil kalthshint lth gudung, waigien hin kwunan il shu-āwon.

14. Waigien tlistleuon la il keng-āwondlu, Istīidū ang-a lth ga liplētgasgai agung kendū, hin la il shudāwon. Waigien hawun il istalūgundān il shkwunagulthāwon.

15. Waigien tl swon, tlistleuon il ngīstlas il kang-andlu, silthgang il stēlan gien, kwünan Shanung-itlagadas ā il kil-lāgan ;

16. Waigien il stai hetgu il tīgūstlū-aian gien, la à il kil-lāgan; waigien lā-ū Samaria haadāgan.

17. Gum gu tl tlāalth shkwūnagulthangüja? gitlan tlou tl tläalth-swonshung-gü ijung?

18. A gu tlan anung tliga kulat haadas swonan Shanung-itlagadas $\bar{a}$ kil-lagai un 
stîlthūja? waigien hin Jesus hanglthang showon.

19. Giā ang-a lth kaid; dung yetdas alth ũ dung ligulgung, waigien hin la il shudaian.

20. Waigien tlistleuon Shanung-itlagadas gia kingdomgai katlagai da Pharisee haade la alth kianang-andlu, hin hang-lthang tla il shudaian, Shanung-itlagadas gia kindomgai katlasdlu, gum kang-gang-ashang;

21. Gwokeng, adlun ijung! Waudlun ijung! gum hin tl shu-ung-ashang; Shanung-itlagadas gia kingdom-gai dalung kalthi $\bar{a}$ isis altha, hin il shouon.

22. Waigien hin ga disciplesgasgai il shudaian, Shantlanai waudlu ëlthsdlu, nung haada Git gia shantlanai swon kang-gai da dalung gudung-ashang, waigien gum dalung keng-ungashang.

23. Gwokeng, waudlun ijung! hin dalung tl shudas gien, Gwokeng, adlun ijung! hin ishin dalung tl shudāashang; gum tl istiidangüga, waigien gum tla dla tl istalang-ūga;

24. Yanai kāan ai sta sē-au-ūlthdas gien, wau hanltha ga otgēlthdalguns ging-an, nung haada Git gyang shantlanai gut etishang.

25. Waigien wau konast gin shing-idsa kwon il ānduns gien, adshi gūdista kang-gai ishin la ga gwau-gulthshang.

26. Waigien Noah nūt tliku gin edan gingan nung haada Git nüt ishin edishang. 
27. Noah arkgai ai kudsaian shantlanai keu tl lthdanūs gien, ishin gin tl nilgang-an, ishin tl jada-nēlths gien, ishin tl lā-inēlgang-an, waigien gīlthīdan, waigien tl tla-hīlū-ōdsou-on. 28. Lot nūt ishin wai ging-an edan; tl lthdanūs gien, ishin gin tl nilgang-an, gin tl dahwos gien, ishin gin alth tl giadahū-gang-an, gin tl tldsas gien, ishin nuh tl tloulthagang-an;

29. Waigien Lot Sodom ilnagai sta kāgwaulan shantlanai ai das isgien brim-kwau-e ishin sha-tligai sta gū-aian, waigien tl tla-hīlūòdsou-on ;

30. Nung haada Git kang-gēlungkasas shantlanai ai wai ging-an etishang.

31. Gai shantlanai ai nas ing-gu nung isis gien, nai à gin lang-a isisdlu, akwon 1th gin ung-a il isdie un gum il kāalthanda; waigien kwulthkotis à nung isisdlu, akwon lth la ishin gum silthgang stilthanda.

32. Lot jā ai lth kēidū.

33. Hēnung-gang gwīgang kāalthdie da nung guduns $\bar{u} \bar{u}$ gudāashang; hēnung-gang nung gudasdlu tlou, il kogundāashang.

34. Gai sing-yē à-hun tl ilthansida stung tīdan ing-gu gut alth tīans gien, nung swon isdias gien, nung swon tl gūhun isisang dalung ga lth shugung.

35. Tl jāada stung gin hekogūduns gien, nung swon isdias gien, nung swon tl gūhun isisang. 
36. Tl ilthansida stung kwulth-kotajai $\bar{a}$ isisang; nung swon isdias gien, nung swon tl gūhun isisang, hin Jesus shouon.

37. Gitlānu, Shalàna? waigien hin hanglthang la tl shudaian. Giāgu tl kōt isis, gai gu ōt ishin gudung-alth gudā isisang, waigien hin tla il shudaian.

\section{Chapter 18.}

WAIGIEN kēgang alth tla ga il kēgang-an, singlthgang tl gushugis gien, gum tl ahgulthang-ai una;

2. Lana a-swonshung gu nung ging-kilisalang-lēga ijan, waigien gum Shanung-itlagadas ga il lthwaugans gien, tl haada alth ishin gum il ai-ū-ang-an ;

3. Waigien gai ilnagai gu nung dsūlthin-ga ijan; waigien la un il katla-hunsgadan gien, Di nung tloulth-dāung-gun alth di da tlāad, hin il shugang-an.

4. Waigien kwai-umdsiwun gum ging-an ūgai da il gudung-ang-an; waigien waudlā hin il gudang-an, Shanung-itlagadas ga gum di lthwaugans gien, tl haada alth ishin gum di ai-ū-uns kianan,

5. Anung dsūlthin-gas di gudung-ai lthkwidadas alth la da lth tläadshang, waigien di un il katlagis ai gum di ahgulthang-ai una, hin il gudang-an. 
6. Tliku nung ging-kilisalang-lëga dāung-as shouon lth gudung-ī.

7. Waigien gum gu Shanung-itlagadas ga kenstaian da ung-a tlāadang-ash, shantlan wautliwon isgien al wautliwon ishin la ga tl sailth-kiāgans gien, tla un il kung-gudung-as altha?

8. Hawidan tla da il tlāadshang dalung ga lth shugung. Nung haada Git katlasdlu tl gu, il kil tl yetdas il kēi-āash? waigien hin Shalana shouon.

9. Itil ĩ lāgung, tl kulat wautliwon tlou dāung-ung, hin ga gudang-an-gai ga adshi kēgang-ai alth il kēgang-an;

10. Tl haada stung singlthgang gushue un temple-gai ā istal-lthaian; nung swon Pharisee haadāgan gien, nung swon ishin giashouganung-lēgāgan.

11. Nung Pharisee haadas giāang-an gien, hin gudung-ung alth singlthgang il gushou-on, Shanung-itlagadas, gum tl haada kulat gingan di edans sing dung ā lth kil-lāgung; tla ga ging-kōnung-gung-usgai isgien, kwolth-dālēalung ishin, gū-inēl king-gogung-ai ga tlahōstagung-usgai ishin, anung giashouganunglēgas ging-an ishin gum di edang-gung.

12. Sundiga shoshgu gut lth kēsal-stunggung-gung; gin lth dāēlths wautliwon tich templegai ga lth isdagung-gung, hin il shouon. 13. Nung giāshouganung-lēgas tlou lthkiā- 
gwau āgiwi giāang-an, waigien gum shi ga kēilthie da il gudung-ang-an; hing-an kunung il shkwūdaian gien, Shanung-itlagadas di kalthshint lth gudung, di dāung-as altha, hin il shouon.

14. Nung swon telg Shanung-itlagadas unis kalthshint gudang-an dalung ga lth shugung, waigien nai à ung-a il kāalan; shā ung nung edas $\bar{u}$ hèt èlthses altha; het ung nung edasdlu tlou, sha èlthishang, hin Jesus shouon.

15. Waigien gitalung kutilas ishin la a tl isdaian, tla ing-gu il tldulthdie una; waigien tlistleuon ga disciplegasgai kang-andlu, tla il stidāwon.

16. Waigien Jesus tla un ai-ang-an gien, hin il shouon, Akwon lth tl āknutilas di à istald $\bar{u}$, waigien lth gum tla $\bar{a}$ stugadang- $\bar{u}$, Shanung-itlagadas gia kingdomgai hin ga etsgai gia isis altha.

17. Yenkien dalung ga lth shugung, Gum nung āknutsū ging-an Shanung-itlagadas gia kingdomgai nung isdāansdlu, gum ai il kudsang-ashang, hin il shouon.

18. Nung Itlagada las, di hēnung-a-swonungai un gūshū lth isdāashang? waigien hin nung itlagada la alth kianang-an.

19. Gushintlou, Nung Lā, hin di dung kyādang, gum nung swonshung-hun lā-unggung, tlan Shanung-itlagadas swonan-a.

20. King-goguns un dung ūnshīdung, Gum 
tl gin kulda-ung-ga, Gum tl haada tl tiiiangga, Gum tl kwolthda-ung-ga, Gum nung alth tl kilthgadung-ung-ga, Aung-ung ga tl guitgìda, ou-ung ishin, waigien hin Jesus il shudai-an.

21. Di dlailāgun sta-hun adshi wautliwon duman lth keng-ginni, waigien hin il shouon.

22. Waigien tlistleuon Jesus gudang-andlu, Gin swonshung gum hawun dung isdang-gung; gin dung daas wautliwon alth gia-dah-ū, waigien lth ka kong-ushgitaigasgai da tlāad, waigien sha-tligai gu gin kwoyā dung da-ēlthishang; ütla, di dla lth kā, hin la il shudaian.

23. Waigien tlistleuon adshi il gudangandlu, il gudung-ai sti-emonan, il gin-lthing-āeuonan altha.

24. Waigien tlistleuon Jesus il kang-andlu, hin il shouon, Ga ginlthing-asgai Shanungitla-gadas gia kingdomgai ai isdsie yenkien shing-idsāashang!

25. Nung ginlthing-a Shanung-itlagadas gia kingdomgai ai kudsie telg, camel stlinkōsi ai kashginai yang-alang, hin il shouon.

26. Gìshd tlou kogunāsīni? waigien ga gudang-an-gai hin shouon.

27. 'Tl haada gum gin isdalthing-ans $\overline{\mathrm{u}}$ Shanung-itlagadas isdalthing-āgung, waigien hin il shouon.

28. Gwokeng, gin wautliwon talung tustlagun, waigien dung dlā talung istalgun, waigien hin Peter shouon. 
29. Waigien hin tla il shudaian, Yenkien dalung ga lth shugung, Nai isgien, jä-ung ishin, kwai-alung ishin, yãalung ishin, gitalung ishin, Shanung-itlagadas gia kingdomgai agunan nung tustlasdlu,

30. La ū wēt kwunan wau telg da-ēlthishang, waigien tliga isangkasas gu il hēnung-aswonung-ashang, waigien hin tla il shudaian.

31. Waigien ga tläalth wangu stunsgai un il ai-ang-an gien, hin la il shudāwon, Gwokeng, Jerusalem à talung istal-lthangkashang, waigien ga prophetgasgai gin kālang-an wautliwon ging-an nung haada Git un etishang.

32. Tliga kulat haade ga la tl isdāases altha, waigien la alth tl kilthnans gien, ishin la tl tloulth-dāung-as gien, ishin la gwi tl lthtāgwong-ashang;

33. Waigien la tl shkiduns gien, ishin la tl tīiā-ashang; waigien shin lthonulthīlthsdlu housen il kalthū-ashang, waigien hin la il shudāwon.

34. Waigien adshi wautliwon gum un il ūnshi-dang-āwon; waigien adshi gushue la sta salgaāwon, waigien gin il shudaian da il kētusāwon.

35. Waigien Jericho un il dōng-ēlandlu, nung hang-kaga gin ginung-ai un keu-e in kou-aian;

36. Waigien tl shkül-euon la gūt istals il gudang-andlu tliku gin etgai da il kianang-an. 
37. Waigien Jesus Nazareth haadas kāang la ga tl shouon.

38. Jesus, David Git dung is, di kalthshint lth gudung, waigien hin kwunan il shouon.

39. Waigien tla kōnast ga istalan-gai il stidaian, kilthwol il itsgai una; David Git dung is, di kalthshint lth gudung, waigien hin kwunan wau telg il shouon.

40. Waigien Jesus giāagan gien, āgang la tla il isdā-alan; waigien tlistleuon il āanē-landlu,

41. Gūsh dung un th isdie da $\overline{\mathrm{u}}$ dung gudung-gung? hin la alth il kianang-an. Shalana, di hung-i helthilthgie da $\bar{u}$ di gudunggung; waigien hin il shouon.

42. Akwon lth dung hung-i helthilthgada; dung yetdie $\bar{u}$ dung kogundang, waigien hin Jesus il shudaian.

43. Waigien hawidan gin il keng-īdan, waigien la dlā il kāgan gien, Shanung-itlagadas $\bar{a}$ il kil-lāgan; waigien tlistleuon tl haada wautliwon kang-andlu, Shanung-itlagadas à tl kil-lāgan.

\section{Chapter 19.}

WAIGIEN Jericho ilnagai ai il kudsaian gien, kalthgut il kagan.

2. Waigien nung ilthing-a Zacchæus hin kya gu ijan; waigien giashouganung-lēalung: un il itlagadagan gien, il ginlthing-āgan. 
3. Waigien Jesus nung isis kang-gai da il gudang-an, waigien tl shkūlas alth odō il eshgaian, il dsūî-kwau-anan altha.

4. Waigien tla kōnast il odan gien, la il kang-gai un sycamore lthkai gwī il kulthaian; gai gut il ka-ungkashagan altha.

5. Waigien tlistleuon Jesus gu katlagandlu, shi ga il kēilthaian gien, Zacchæus, hawïdan lth käalth, aiat dung gia nai $\bar{a}$ lth isangkasas altha, hin la il shudaian.

6. Waigien il kutalan gien, guảung-ai lā dung-alth kwulthgang la il isdaian.

7. Waigien tlistleuon tl kang-andlı, tl gīdāung - ōdsou - on, Nung îlthing-a dāung-a kwulth il itsgai un ai il kudsang, waigien hin tl shouon.

8. Waigien Zacchæus giāagan gien, Gwokeng, Shalana, ga kong-ushgitaigasgai ga gin lth dāas gūdlū shäawă lth isda-ung-kashang; waigien ging-gang-an nung haada lth giashoudaiasdlu, wau telg etgai stunshung dlū la ga silthgang lth isdäashang, waigien hin Shalana il shudaian.

9. Waigien hin Jesus il shudaian, Aiat adshi nai $\vec{a}$ nung is kogun-gung, la ishin Abraham gitalung swon isis altha.

10. Nung haada Git ga gougasgai da kens gien, ishin il kogundie un katlas altha, hin la il shudaian.

11. Waigien hawun adshi tl gudung-gun- 
dān, kēgang alth ishin il kēgang-an, Jerusalem un il ãanas gien, ishin Shanung-itlagadas gia kingdomgai hawīdan kang-gēlthshang tl gudang-an altha.

12. Waigien hin il shouon, Nung itlagada ken-ung un kingdom isdas gien, silthgang il stelgai un tliga jīng-a à ijan.

13. Waigien gitsadalung shu sta tl tlāalth un il ai-ang-an gien, pound tlāalth la ga il isdāwon, Lth katlagai keu lth adshi gudista tla-kāwong, waigien hin la il shudāwon.

14. La kwulth-it ga nāansgai tlou la telg. kang-an gien, la dla gushou tl hundsūdaian, Gum anung îlthing-a isis itil un itladagai ga itil stalthang-gung, waigien hin tl shouon.

15. Waigien tlistleuon kingdomgai il isdaian gien, silthgang il stēlandlu, a ga gitsada ga dālē il isdaian un il lthai-i-halan, gudista gin il tla-kāawondlu tlistleu la gwi kāalthawē un il ūnshidai una.

16. Waigien nung swon la un katlagan gien, Nung itlagadas, dung gia poundgai gwī pound tlāalth kāalgun, hin il shouon.

17. Hou-a; gītsada lā dung ijung; gin gūdsū-dsū duman dung kang-gai lagun alth, lana a-tlāalth un dung lth itlagadēlth-dang, waigien hin la il shudaian.

18. Waigien nung swon ishin la un katlagan gien, Nung itlagadas, dung gia poundgai gwi pound tlēlth kāalgun, hin il shouon. 
19. Akwon lth dā ishin lana a-tlēlth un ung itlagadādi, waigien hin la ishin il shudaian.

20. Waigien nung kulat ishin katlagan gien hin il shouon, Nung itlagadas, $\bar{a} \overline{\mathrm{u}}$ dung gia poundgai ijung, giang- $\bar{u}$ ung-a $\bar{u}$ alth duman lth keng-gun;

21. Dung ga di lthwaugagun, nung ilthinga skulthja dung isis altha; gum hēt gin dung isdans hun dung isdas gien, gum gin dung tldsans hun ishin dung tliidung-gung, hin il shouon.

22. Waigien hin la il shudaian, Tliku dung shus alth tliku dung ets dung lth kendāashang, nung gitsada dāung-a isis. Nung ilthing-a skulthja di isis gien, gum hèt gin lth isdans hun lth isdas gien, gum gin lth tldsans hun ishin lth tliidung-us un dung ūnshidun;

23. Gushintlou gum dàla-nai ai dālē dīna dung isdang-ūjang, waigien lth katlasdlu, dālē isgien, wau gwī kāalthsi ishin lth isdashgaiahung-gung? hin la il shudaian.

24. La sta lth poundgai isdū, waigien lth pound tlāalth nung dāas ga isdū, hin la kwulth ga tljūgiaang-an-gai il shuduian.

25. Nung itlagadas, pound tlāalth il dāgīgung, waigien hin la tl shudaian.

26. Gin nung dāasdlu, la ga gin isdiāashang; gum gin nung dāansdlu tlou, gin il dāas huu la sta isdiāashang dalung ga lth shugung.

27. Waigien di telg ga kang-an-gai un lth 
itlagadagai ga gwou-aian lth althgwĩ isdū anga lth di hang-gu tl-dū, hin il showon, hin Jesus shouon.

28. Waigien tlistleuon adshi il shudagiggandlu, tla kōnast il kaidan gien, Jerusalem a il kulthai-an.

29. Waigien tlistleuon Bethphage isgien Bethany ishin tldou Olives tldäwe hin kyagan gu ijan un il döng-ēlandlu, gyang disciplesgai stung il kilistīidan gien hin il shouon,

30. Dalung hanltha lana isis $\bar{a}$ lth istiidu ; waigien ai dalung isdsasdlu, geudan giti gum hawun gwau tl kāwung-ung keutl-dajā dalung keng-ashang; la aista lth tläadì ang-a althgwi il isdū-wong.

31. Gushintlou la aista dalung tlä-ëdung? waigien hin dalung nung shudasdlu, Shalana ĩ. la ga stalthang, hin tl shü-wong; waigien hin la il shudäwon.

32. Waigien la ga kil-istiidan istīidan gien, tliku gin la ga il shudäwon ging-an gin il keng-āwon.

33. Waigien hawun geudan-gai giti aista il t]äadiigundān, Gushintlou geudan-gai gîti aista dalung tläēdung? hin la ga dāagan-gai il shudāwon.

34. Shalana ū la ga stalthang, waigien hir il shū-āwon.

35. Waigien Jesus ā la il isdāwon; waigien gin tl giandas geudan-gai giti ing-gwi tl 
danlthaian gien, Jesus la ing-gu tl koudaian.

36. Waigien hawun il kāgundān, gin tl giandas keu-e ai tl duntang-an.

37. Waigien il dōng-ēlandlu, Olives tldāwe kwul gu il ijandlu, ga disciplegas shkũlas wautliwon lthong-gwila dugwia tl kang-an wautliwon alth tlika tl gwūlā-ēdan, waigien. Shanung-itlagadas ā kwūnan tl kil-lā-êdan;

38. Nung King-ga Shalana kye $\bar{a}$ katlas $\bar{u}$ yenki lāgung; akwon lth shatligai gu gudungai dlaia isdū ang-a sha àgwīgai un yägudung$\bar{a} d u$, waigien hin tl shouon.

39. Nung Itlagadas, gyang disciplesgai lth stidi, waigien hin ga shkūlas shu sta Pharisee haade ilthi il shudaian.

40. Ashgai tlan gushusdlu, kwau-e ī gūshuiashang dalung ga lth shugung, waigien hin hanglthang il shouon.

41. Waigien tlistleuon il dông-elandlu, ilnagai il kang-an gien, kalthshint il sailthaian,

42. Waigien hin il shouon, Gin alth t] gudung-ai dlaias un wēt dung ünshidasdlu, yenkien dung un lashgaia-hung-gung! wèt tlou lung: sta salgāgung.

43. Dung telg ga kensgai dung odō gin lthetīgadulths gien, dung odō un $t 1$ gilthilthdas gien, dung tl keng-kēäwashang,

44. Waigien dung isgien dung gitalung dung $\vec{a}$ is dung-alth tligai ing-gu tl kêi-dlish- 
gutses shantlanai isisang; waigien gum kwan kē-swonshung un dung à gut ing-gu tl kēIthindadang-ashang; dung un nung katlagundlı gum un dung unshidang-gun altha, hin il shouon.

45. Waigien templegai ai il kudsaian, waigien a gin alth tl giadahwos kya ga il dangwau-ēdan,

46. Giagun nai singlthgang gushou nai $\bar{u}$ isisang, linnu kālung-āgung; dalung tlou kwolthdālēalung gia dīnai un tloulthagun, waigien bin tla il shudaian.

47. Waigien shantlan wautliwon gut templegai à gin tla il shkotadagang-an. Waigien liplēt un ga itlagadasgai isgien, tukālang-lēalung ishin, tl haada un ga itlagadasgai ishin, il tīie da gudang-an;

48. Waigien gum tliku la tl edalthing-e kang-gang-an; tl haada wautliwon il kil gudung-ai da gudung-euonan altha.

\section{Chapter 20.}

WAIGIEN shantlan swonshung gut, hawun templegai ā tl haada gin il shkotadas gien, ishin gialthang las alth tla geu gu il gushugundān, liplēt un ga itlagadasgai isgien tukā-lang-lèalung ishin, ga kèasgai dung-alth la un istlagan;

2. Waigien tl gushouon, Adshi lth itil ga 
shüdi; gūsh dugwīgai alth $\bar{u}$ adshi dung isdas gien, gishd ishinan $\bar{u}$ adshi dugwigai dung ga isdaiang? waigien hin la tl shudaian.

3. Ltha ishin gin da dalung alth kianungashang; di ga lth shūdū ;

4. John gia baptism-gai gu shatligai sta ija? tl haada sta gwau? waigien hin hanglthang tla il shudaian.

5. Waigien edē tl kāūgaian, Shatligai sta, hin talung shusdlu, Gushintlou gum la dalıng: yetdang-üjang? hin il shu-ashang.

6. Waigien, Tl haada sta-a, hin talung shusdlu, tl haada wautliwon itil kunlthūashang; John prophetgagan un tl unshits altha, hin gu tl shudaian.

7. Gum tliji sta isi un itil unshidang-gung, waigien hin hanglthang tl shouon.

8. Ltha ishin nung gia dugwigai alth adshi isdas gum dalung ga lth shudang-ashang, waigien hin Jesus tl shudaian.

9. Waigien adshi kēgang-ai alth tl haada ga il kēgang-īdan; Nung îlthing-a wine ānē Ithkai kwulth-kotajai tloulthaian gien, tōkānlëalung il shildadaian, waigien tliga kulat a il kaidan, gu il is-hunsgadai una.

10. Waigien ān nūtgēlandlu ga tōkanlēgasgai $\bar{a}$ nung gitsada il kil-kaidan, wine ānē kwulthkotajai sta ān la ga tl isdie una; waigien ga tôkanlēgasgai il shkidang-an gien, ān àdan la tl kaidaian. 
11. Waigien housen nung gìtsada $\bar{a}$ il kilkai-dan; waigien la ishin tl shkidang-an gien, la tl tloulth-dāung-euonon, waigien ān àdan la tl kaidaian.

12. Waigien housen nung il kilkaidan; waigien la ishin tl tligaidaian gien, kya ga la tl dang-an.

13. Gushinu lth etishang? gitang kwoyasi à lth kilkaidshang; la ga tlou tl gūtgutshahung-gung, waigien hin wine ānē kwulthkotajai un nung itlagadas shouon.

14. Waigien tlistleuon ga tōkanlēgasgai il kang-andlu, la edē gut ga tl kāūgaian, A ī la silth nalthing-ai ijung; la talung tiiadsun, adshi kwulthkotajai itil gia isēle una, waigien hin tl shouon.

15. Waigien wine ānē kwulthkotajai sta la tl dang-an gien, la tl tìi-aian. Gushinu waigien wine ānē kwulthkotajai un nung itlagadas tl et-dāashang?

16. Il katlas gien, a ga tôkanlēgas il hīlūdāashang, waigien wine ānē kwulthkotajai tl kulat ga il isdāashang, hin Jesus shouon. Waigien tlistleuon tl gudang-andlu, Akwon lth gum ging-an tl edang, hin tl shouon.

17. Waigien tla gwi il kēaian gien hin il shouon, Nuh tloulthalē-alung kwau dangwaian $\overline{\mathrm{u}}$ nai kwūngut un kudsēlgung, hin kālung-āsi gushinū èdung?

18. Wodshi kwau-e ing-gu nung dlu-issdlu, 
lē dsulthuldashang; nung ing-gwī hun kēwīsdlu tlou, kōī ging-an la ga kudsulthūsdāashang, hin il shouon.

19. Waigien tukālang-lēalung isgien, liplēt un ga itlagadasgai ishin, gai dlu il gitsgīlthdie da gudang-an; waigien tl haada ga tl lthwaugaian; tla edē adshi kēgang-ai il shudaian un tl ūnshidan altha.

20. Waigien la à tl lthotsaian gien, tl haada lā ging-an agung ga etdaian la à lthotsie un tl kil-istīidan, gin il kilshgūdasdlu lang-a tl guduns gien, nung governorgas gia dugwïgai $\bar{a}$ la tl isdie una.

21. Waigien hin la alth tl kianang-an, Nung itlagadas, yenkien gin dung shudas gien, ishin yenkien gin tla dung shkotadas gien, ishin gum tl lthū alth dung ai-ū-uns gien, tliku shanung-itlagadas guduns ging-an gin tla dung shkotadagung-us un itil ūnshidung;

22. Cæsar ga talung giashougung-us gu la? waigien hin la alth tl kianang-an.

23. Waigien gin agunan tl shus un il unnshidan gien,

24. Penny kēshgu lth di kendū. Gīshd da nījung-ai $\bar{u} \bar{a}$ ijung? gūsh k'älung-e ishinan $\bar{u}$ $\bar{a}$ jjung ? hin tla il shudaian. Cæsar gia, waigien hin tl shouon.

25. Cæsar gia isisdlu lth Casar ga isdū, waigien Shanung-itlagadas gia isisdlu lth Shanung-itlagadas ga isdū, waigien hin tla il shudaian. 
26. Waigien tl haada hang-gu gum tliku gin il ging-kilshgūdalthing-e kang-gang-an; waigien hanglthang il shouon un tl kuldung-àgan gien, gum tl kīlang-an.

27. Waigien Sadducee haade ilthi la un istlagan, gum kotal sta tl kalthougadung-ungashang ga gudang-an-gai-a; waigien hin la alth tl kianang-an,

28. Nung itlagadas, Nung ilthing-a kwai kotulthsdlu; waigien il jā gum git-kaiwons gien, il dōn il jā isdie lagung, waigien kwaiung un tl ăkutila il kaiwie una, hin Moses itil un kālang-an.

29. Waigien tl dsikwau gut alth istalgāgun; waigien nung kwai-as jadanēlgun gien, gitkai ädan il kotulgun;

30. Waigien la gūst nung kugas ishin il jã isdagun ;

31. Waigien la gūst lana ishin il isdagun ; waigien wai ging-an dsikwau-hun git-kai ādan kotulthgagun.

32. Waudla nung jadas ishin kotulgun.

33. Kotal sta tl kalthougadunsdlu, gīnas jā $\bar{u}$ il isisang? dsikwau-hun il inādalgun altha, hin la alth tl kianang-an.

34. Waigien hin Jesus tl shudaian, Adshi hēt-tligai haade jada-nēlths gien, ishin tl lainēl-gung-gung ;

35. Althsgai tligai gu itsgai un isgien, kotal sta kālthūe un ga lasgai ishin, gum jada- 
nēlthans gien, ishin gum lā-īnēlgung-unggung ;

36. Gum housen tl kotulthgalthing-anggung; angels ging-an tl ets altha; waigien Shanung-itlagadas gitalung $t \mathrm{l}$ isgien, kotal sta kalthūe gitalung ishin tl ijung.

37. Waigien Moses gin Lthkai edē kālangandlu, kotal sta tl kalthougadung-gung-us tla il kendaian, tlistleuon Abraham Itlagĩda, Isaac Itlagīda, Jacob Itlagīda, hin Shalana il kyādaiandlu-a.

38. Gum ga kotulthgiasgai un il Itlagadanggung, ga hēnung-asgai un tlou il Itlagadagung; tl wautliwon la hang-gu hēnung-as altha, hin Jesus tl shudaian.

39. Nung Itlagadas, gwaulthang-an dung shugung, waigien hin tukälung-lēalung ilthi hanglthang shouon.

40. Waigien waudla gin da la alth kianungai ga tl lthwaugalan.

41. Waigien hin tla il shudaian, Gushintlou nung Christgas David git ising tl shugung?

42. Sōolgūst lth di kwulth kou-i, dung telg ga kensgai dung stai keusi un lth isdie keu-a, 43. Hin Shalana giagun Shalana-gai shudaian, hin David kodan Psalms kōgīnē a shouon altha.

44. David Shalana hin il kyādasdlu, gushintlou il git il ijung? waigien hin tla il shudaian. 
45. Waigien tl wautliwon geu gu hin gyang disciplesgai il shudaian,

46. Tukälang-lëalung sta lth ga gütgung-ū, kōdats jinda hetgu istalgung-ai da tl guduns altha, gya gu tl wahdas gu ishin tla hung-a tl kwah-gulthdas gien, synagogue-nagai a ishin aulthgahung-ū ladsilthīs isgien, lagunang gu ishin aulthgahung-u ladsilthīs tl kwoyädagung-gung ;

47. Ga dsūlthin-gas gia ginagai tl kwolthdaódsūs gien, tl lang tla un tl gudung-ai un ishin singlthgang gushue ung-a tl jindadagunggung; ashgai $\overline{\mathrm{u}}$ tl kulat telg tlū-unshkotiāashang, hin tla il shudaian.

\section{Chapter 21.}

WAIGIEN il këilthaian gien, ga îtlagadasgai dāla ôdē ai dālē ung-a isdas il kang-an.

2. Waigien nung dsülthin-ga kong-ushgitaiga mite stung wē isdas il kang-an.

3. Waigien hin il shouon, yenkien dalung ga lth shugung, Tl wautliwon telg anung dsülthin-ga kong-ushgitaigas wē isdagun;

4. Atltas wautliwon gin kwon tl daas tich wē isdagun altha; la tlou kong-ushgitaigaskianan, gin alth il hēnung-as wautliwon wē il isdagun, hin il shouon.

5. Waigien kwau kwōyã alth isgien, gūdì 
gin $\mathrm{tl}$ isdaian alth ishin templegai las edē $\mathrm{tl}$ īlthi g'üshūgundān, hin il shouon,

6. Adlun gin dalung kens hilliashang, waigien gum adlun kwau kē-swonshung-hun gūt ing-gu isang-ases shantlanai isisang, wantliwon gūdista dang-wiāashang, hin il shouon.

7. Nung Itlagadas, gīshindū adshi isisang? waigien adshi isangkasasdlu, gūsh kang-ūdsada $\bar{u}$ isisang? waigien hin la alth tl kianang-an.

8. Waigien hin il shouon, Duman lth agung keng- $\mathrm{u}$, waigien gum dalung tl al-istīidg $\bar{u} d a n g-$ ung-ai una; tl kwon di kyē à istlas gien, Lā-ī di ijung, hin tl shūs gien, Time-gai āanāgung, hin ishin tl shū-ases altha; gum tla dlā tl istīidang-wong.

9. Waigien tlistleuon gu-isda isgien, gudan lthkwida alth gialthing-e ishin dalung gudunsdlu lth, gum lthwaugulthang-wong; adshi is-tlagang-ai las altha; hỉkwon gum hawun tlan gin elthang-ashang, hin il shouon.

10. Itan hin tla il shudaian, Gwaigang alth gwaigang gut hanltha elths gien, kingdom alth ishin kingdom gut hanltha elthishang;

11. Waigien tliga yildung-euons gien, tliga kwon ai ishin tou hīlūs gien, ishin itskāashang ; waigien gin ga Ithwaugigas gien, sha-tligai sta ishin gin kang-ūdsada euonda isisang.

12. Waigien a gin is wautliwon konast dalung tl gitsgilthdas gien, gin shing-idsa dalung tl ändung-dāashang, waigien synagogue-nagai 
$\bar{a}$ isgien kéchitnai $\bar{a}$ ishin dalung tl isdãashang, waigien ga king-gasgai isgien, ga governorgasgai hang-gu ishin di kyē agunan dalung tl isdāashang.

13. Gin unsta tl shuguns ging-an dalung un etishang.

i4. Althji alth tliku hanglthang dalung shūases gum wau kōnast adō dalung kwūlthilthang-ai un lth gudung-ung elthgīdu ;

15. Gushou isgien, kutung-a ishin dalung ga lth isdāases altba, waigien dalung hanltha ga etsgai wautliwon gum tliku hanglthang shoulthing-e kang-gans gien, dalung kil-kāalthdie odō ishin tl eshgāashang.

16. Waigien dalung aung-alung isgien, dalung kwai-alung ishin, dalung tō-alung yiki ishin, waigien tl kulat dalung tō-aldas hun, dalung unsta shu-ashang; waigien tl kil agunan dalung îlthi tl tldāashang.

17. Waigien di kyè agunan tl haada wautliwon dalung telg keng-ashang.

18. Waigien gum dalung kuts ou sa-swonshung-hun gū-ung-ashang.

19. Dalung gūtgulthdsū-wosdlu, dalung althandai dalung gwī ging-kāalthishang.

20. Waigien tlistleuon soldier haade kwon Jerusalem odō èlths dalung kensdlu, itan hawìdan ilnagai gūases un lth ūnshitwong.

21. Itan Judæa gu tl isis tldāwē ā knūstie lagung; ilnagai kalthgut tl isis ishin kya ga 
itsgai lāgung; ilnagai kyagust ga nāansgai ishin gum ai isdsie lā-ung-gung.

22. Ashgaitlin-unshkotashantlanai isis altha, gin kālung-as wautliwon wai ging-an etgai una.

23. Ga dulthgiasgai isgien, ga tutlindasgai un ishin ashgai shantlanai gut gin ga lthwaugi-gāashang! Tliga gut gudan lthkwida euon isgien, ashgai haadgai un ishin tl kalthiyildung-ases altha.

24. Waigien shich alth tla tl tldas gien, tliga kulat wautliwon ai tla tl haldang-adāashang; waigien tliga kulat haada Jerusalem ai tunanung-ashang, tliga kulat haada gia timegai tlan elgai keu-a.

25. Waigien jūyē ā isgien, kōng-ai $\bar{a}$ ishin, kailthdai à ishin kang-ūdsada isisang; waigien hēt-tligai gu gwaigang-ai gudung-ai lthkwīdāashang, tung-ai isgien ai-a-we ishin dumdagas alth tl gudung-ai hīluashang;

26. Waigien tl lthwaugas isgien, hēt-tligai gu gin isangkasas keu tl khatsus ishin tl gingkotulth-ga-kwūndāashang; sha-tligai dugwīgai yildung-ases altha.

27. Waigien itan nung haada Git gin dugn ia alth isgien, gin un yāgudung-a alth ishin yan kälthi a katlas tl keug-ashang.

28. Waigien tlistleuon a gin isis is-tlagañgidsdlu lth, kēitlāu ung-a sha-ung-ou-wong; dalung kogunai dōng-ëlthses altha, hin tla il shudaian. 
29. Waigien kégang alth tla ga il kègangan; Fig lthkai isgien, gin lthkai wautliwon ishin lth keng-ī ;

30. Tlistleuon hyilē kōstusdlu, dalung kenggung-gung, waigien sing kīna dōng-elths un dalung unshidung-gung.

31. Waigien dalung ishin, tlistleuon a gin isis dalung kensdlu, Shanung-itlagadas gia kingdomgai dong-êlths un lth unshitwong,

32. Yenkien dalung ga lth shugung, Gin wautliwon elthgïgai keu, adshi guidista kanggai gum tlan elthang-ashang.

33. Sha-tligai isgien, hêt-tligai ishin tlan elthshang; di kil tlou gum tlan elthang-ashang.

34. Duman th waigien agung keng-u, waigien lthdanū-euon isgien, lumga-euon ishin, adshi het-tligai gudan lthkwidie alth ishin gum dalung gudung-ai stahū-uns gien, gum shkum ging-an gai shantlanai hawidan dalung $\mathrm{g} w i \overline{\text { isang-ai una; }}$

35. Wai ging-an hēt-tligai ashkwau-hun gut ga nāansgai wautliwon ing-gu isises altha.

36. Waigien lth shanlthotsgi-u ang-a singlthgang güshūu, gin isangkasas wautliwon sta dalung koguns gien, nung haada Git hang-gu dalung tljügiãang-ai una, hin il shouon.

37. Waigien shantlan wautliwon ai templegai $\bar{a}$ gin tla il shkotadagang-an; waigien sing-yā wautliwon gut il kāgwūlths gien, tldou Olives hin kyāgan gu il aldagang-an. 
38. Waigien tl haada wautliwon sing-ai un il kil gudung-ai un templegai $\bar{a}$ la un istlagang-an.

\section{Chapter 22.}

W AIGIEN shibli tlukulthdāive gū hunt ladōng-ēlan.

gunung-ai, Passover hin kyāgang-an,

2. Waigien liplet un ga itlagadasgai isgien, ga tukālung-lēgasgai ishin tliku la tl tiitljoulthing-as edē tl kāügaian; tl baada ga tl lthwaugaian altha.

3. Waigien ga tlāalth waugu stunsgai swon, Judas Iscariot hin kyāgan, ai Satan kudsaian.

4. Waigien il kaidan gien, liplêt un ga itlagadasgai isgien, ga captain-gasgai $\bar{a}$ ishin tliku tla ga la il isda-tljoulthing-as il kilūlthaian.

5. Waigien tl gudung-ai lāgan, waigien dāla la ga $\mathrm{tl}$ isdasang-an.

6. Waigien il gwūlāgan, waigien tl shkūlas hung îlā tla ga il isdie il isdagūdang-an.

7. Waigien shibli tlukulthdāwe gū shantlanai ijan, tlistleuon passover lumadōgai tl tīie lāgandlu-a.

8. Waigien Peter isgien John ishin il kilistiidan, Passover-gai lth itil un tla-elthgiāī, talung tugai una, waigien hin il showon.

9. Gitlanu talung tla-elthgigai da dung gudung-gung? waigien hin la il shudāwon. 
10. Waigien hin la il shudāwon, Tlistleuon ilnagai ai dalung isdsasdlu, nung ilthing-a ga jijūs un dalung istlāashang; nuh ai il kudsas ai lth la dlā isdsawong.

11. Gitlānū lthdanou-roomgai ijung, a gyang disciplesgai alth passovergai th tugai una? hin nung Itlagadas dung shudang, hin nung nuh-lēgas dalung shudie lāgung.

12. Waigien nuh-shasi $\bar{a}$ room euon tlaelthgīgas dalung il kendāashang; gai à lth tla-elthgiwong, waigien hin la il shudāwon.

13. Waigien il istīidāwon gien, tliku la il shudāwon ging-an etsi il keng-āwon; waigien passorergai il tla-elthgī-āwon.

14. Waigien tlistleuon waudlu ëlandlu, ga apostlegasgai dung-alth il kāwon.

15. Di waugung-ai kōnast, adshi passovergai dalung alth tugai da di gudung-euon-gun;

16. Shanung-itlagadas gia kingdomgai gu ga elthgigai keu, gum housen dalung alth lth tã-ung-ashang dalung ga lth shis altha, waigien hin la il shudāwon.

17. Waigien shkutlang-ū il isdaian, waigien sing il kil-lāgigandlu hin il shouon, Adshi lth isdù, dalung wautliwon lth wausta hükadalū ;

18. Adshi sta Shanung-itlagadas gia kingdom-gai katlagai keu, gum housen vine ānē hou dalung alth lth hütlang-ashang dalung ga lth shus altha, hin il shouon.

19. Waigien shibli il isdaian, waigien sing 
il kil-lägigandlu ai il ginanang-an, waigien la ga il isdāwon, Adshi di lthū dalung un isdias $\bar{u}$ jjung; di à dalung gudanung-ai un lth hin waugung-wong, waigien hin il shouon.

20. Waigien il lthdanügiäwondlu, shkutlangwē ishin il isdaian gien hin il shouon, Adshi shkutlang-wẽ $\bar{u}$ di ai dalung un kwah-yangungkasas shkotaga outla ijung.

21. Waigien gwokeng, di alth nung gia-dahung-kasas stlai adshi tablegai ing-gu di alth ijung.

22. Tliku wau kōnast elthgīgun ging-an, yenki nung haada Git tligwi isang-kasas altha; nung haada di alth giadah-ung-kasas un tlou gin ga lthwaugigaaashang! waigien hin il shouon.

23. Waigien tliku nung et adshi isda-ungkasas da gut alth il kianung-idãwon.

24. Waigien la shū nung itlagadāwos edēishin il käūgāwondlu, gu kilth shontga il gūshuīiāwon.

25. Waigien hin la il shudāwon, Tliga kulat haade un ga king-gas tla un itlagadāgunggung; waigien tla un ga itlagadasgai tla da tläēdlēalung hin kyägung-gung.

26. Dalung tlou gum ging-an etgai lä-unggung; dalung shu sha nung ets tlou akwon dalung un il dönëlë lägung; dalung shu nung îtlagadas ishin dalung un gitsadāgai lāgung.

27. Ginasu sha ēdung? nung lthdanū-wos. gien, il gitsada ishin? gum gu nung lthdanūwos 
sha edang-ūsh ? ltha tlou dalung shu tl gitsadāguns ging-an edung.

28. Dalung ù di edē tl kil-kenskialthk di alth ijuni ;

29. Waigien kingdom dalung un lth elthgidagun, di Aung kingdom di un elthgìdagun ging-an-a ;

30. Giagun kingdomgai gu di alth dalung Ithdanūe una; waigien aulthgahung-ū las ing-gu dalung tlōdas gien, Israel gwaigang-ai t]āalth waugu stung dalung ging-kilisalung-ashang.

31. Simon, Simon, Satan dung isdie da kianung-gun, wheat tl whüstagung-us ging-an dung il isdie una ;

32. Waigien dung sing lth gushugun, dung yet-die gum tlan elthang-ai una; waigien housen dung stilthsdlu lth, kwai-alung tldugwiēlthang, hin la il shudaian.

33. Shalana, kechitnai à dung alth kogai isgien, dung alth kotulai un ishin di elthgīgàgung, waigien hin la il shudaian.

34. Peter, di un dung unshits da dung kudālthōnulē keu, gum aiat shkou ilthing-as kingung-ung-ashang dung ga lth shugung, waigien hin il shouon.

35. Dāla gwau-ale ādan isgien, tou gwaul-ale àdan ishin, stashgaga ādan ishin dalung lth kilistīidundlu gu, gin da dalung kē-ai-a ? waigien hin la il shudāwon. Gāanū, waigien hin il shū-ä̀on. 
36. Waigien hin la il shudāwon, Wet tlou dāla gwau-ale nung isdasdlu, il isdie lāgung, waigien tou gwau-ale ishin; waigien gum shich nung dāansdlu, kodatsai alth ung-a il giadahgai lāgung, waigien shich il dahgai una.

37. Gin dāung-a isdālē-alung alth il kwaiindiāgun, hin kālung-āsi ging-an lth etgai lang dalung ga lth shus altha; tliku di ede gin tl kālang-an ging-an etses altha, waigien hin la il shudāwon.

38. Shalana, a shich tl-stung ijung, waigien hin il shuāwon. Lāgung, waigien hin la il shudāion.

39. Waigien il kāgwaulan gien, tliku il ijang-an ging-an Olives tldāwe à il kaidan; waigien ga disciplegasgai ishin la dlā istalan.

40. Waigien tlistleuon gu il katlagandlu, Gum dalung edē tl kìl-keng-ung-ai un lth singlthgang gūshūū, hin la il shudāwon.

41. Waigien tlistleu kwau tl kātagung-us dlu tla sta il kāgan; waigien kwolong kutsung alth il kou-on gien, singlthgang il gushouon,

42. Aung-a, dung gwūlasdlu lth, adshi shkutlang-wē di sta isdi; waikian lth tliku lth guduns ging-an gum edang; tliku dung guduns ging-an lth et, waigien hin il shouon.

43. Waigien sha-tligai sta nung angel-ga agung il kendaian gien, la il tl-dugwiēlan.

44. Waigien il gudung-ai sti-euon-andlu, 
wau telg kwunan singlthgang il gushouon; waigien il dung-althdaiandlu, ai kwah-tuldangeuon-gung-us ging-an tligai ing-gu lang-a kwah-tuldang-an.

45. Waigien tlistleuon singlthgang il gushouon sta il giāgandlu, gyang disciplesgai un il katlagan, waigien il gudung-ai sti-wos tist il kou-os lang-a il keng-āwon,

46. Gushintlou dalung kogung? kālthū ung-a lth singlthgang güshüü, waigien gum dalung edè tl kil-keng-ung-ai una, waigien hin la il shudāwon.

47. Hawun il gushugundān, tl shkūl-euon istlagan, waigien ga tlāalth waugu stunsgai swon, Judas hin kyagan, tla un kutl-jāwon; waigien Jesus à il kāgan la il shkwūntlie una.

48. Judas, shkwüntla dung-alth gu nung haada Git alth dung giadah-shāūsh ? waigien Jesus hin il shudaian.

49. Waigien la odō ga ijan-gai tlistleuon tliku gin èdangkasas tl kang-andlu, Shalana, shich alth gu tla talung shoshgadash? hin tl shouon.

50. Waigien tl swon liplēt un nung itlagadas gitsada shoshgadan gien, sōolgūistgai il geu wau sta lang-a il shki-otlaian.

51. Kwai lth etdū, waigien hin Jesus hanglthang shouon. Waigien il geu lang-a il tlitlaian gien, lang-a il tla-ngistlaian. 
52. Waigien liplēt un ga ìtlagadasgai isgien, templegai un ga captain-gasgai ishin, ga kēasgai ishin, la hanltha etgai un la un istlagan Jesus hin shudaian, Nung kiwōlthdalēga hanltha etgai un tl istlaguns ging-an gu, shich dung-alth isgien, shkong-ū dung-alth ishin di un dalung istlā-ūsh ?

53. Shantlan wautliwon templegai ã dalung: alth lth ijundlu, gum di dalung gitsgilthdanggin̄i ; waigien dalung gia timegai $\bar{a}$ isis gien, algagai dugwigai ishin ā ijung, hin tla il shudaian.

54. Waigien la tl gitsgilthdaian gien la tl al-kaidan, waigien liplët un nung itlagadas gia nai ai la tl isdaian. Waigien Peter tla dlā āgwī kāgan.

55. Waigien tlistleuon nai kēū gu tl tsanouon gien tl wautliwon gudung-althan tläwondlu, Peter ishin tla shu kou-aian.

56. Waigien hawun tsanu-e otgagai $\bar{a}$ il kougundān, nung jada itan inā il kang-an, waigien la il khatsgilthdaian gien, Unis ishin la alth ijun, hin il shouon.

57. Waigien da il kudaian, Nung jadas, gum la un di ünshidang-gung, waigien hin il shouon.

58. Waigien gum jīng-ang-gundān nung kulat ishin il kang-an, $D_{a}$ ishin tl swon ijun, waigien hin il shouon. Nung ilthing-as, gum tl swon di isang-gung, waigien hin Peter shouon. 
59. Waigien wau sta ashkoshgit swonshangandlu, nung kulat ishin kwūnan gushouon, Yenkien anung ilthing-as ishin la alth ijun, il Galilee haadas altha, waigien hin il shouon.

60. Nung ilthing-as, gum tliku dung shus un di unshidang-gung, waigien Peter hin shouon. Waigien hawun il gushugundan, hawidan shkou-e king-ang-æxn.

61. Waigien Shalana gwī kĩ-aian gien, Peter il khatsgillthdaian. Waigien tliku Shalana shouon ai Peter kēidan, Aiat, shkou ilthing-as king-ung-ai kōnast di da dung kuda-lthōnulthshang, hin la il shudaian-a.

62. Waigien il kāgwaulan gien, il sailtheuon-an.

63. Waigien Jesus ga gitsgadan-gai la alth kilthnang-an gien, la tl shkidang-an.

64. Waigien il hung odō gin tl isdaian gien, Unsta lth shu; gishdü dung shkwudang? hin la alth tl kianang-an.

65. Waigien gin àda kwon la hanltha tl shudaian gien, gushou dāung-a la ga tl isdaian.

66. Waigien itan shantlan-gundān, ga kēasgai councilga un gudung-althan gudā ijan, waigien liplet un ga itlagadasgai isgien, ga tukālanglēgasgai ishin gu ijan; waigien councilgai à ung-a la tl alkaidan ;

67. Nung Christgas dung isdō lth, itil ga shudi, waigien hin tl shour. Waigien hin 
tla il shudaian, Dalung lth shudadōwan, gum dalung yetdang-ashang;

68. Waigien dalung alth lth kianung-dōwan, gum hanglthang dalung shu-ung-ashang.

69. Waigien adshi sta-hun nung haada Git Shanung-itlagadas dugwīgai sōolgūst kou-wauashang, waigien hin tla il shudaian.

70. Shanung-itlagadas Git gu dung isüsh? waigien hin $t \mathrm{l}$ wautliwon shouon. Dalung $\overline{\mathrm{u}}$ lth ising shugung, waigien hin tla il shudaian.

71. Gūsh-un-ū witness ga housen itil stalthang? Talung gudan helthang ai sta il shus gudung-gun altha, waigien hin tl shouon.

\section{Chapter 23.}

WAIGIEN tl wautliwon-hun tljūgiaian gien, Pilate à la tl isdaian.

2. Waigien la hanltha tl gushuīidan, Anung ilthing-as itil gia haadgai shu gudan lthkwīda tloulthas gien, Cæsar ga tl giashougai sta tla il stidas gien, Christ nung king-ga il ising il shus talung kēigun, waigien hin tl shouon.

3. Dung gu Jews haade un king-ga-ūsh ? waigien hin Pilate la alth kianang-an. Dung kil-lthyetdang, waigien hin hanglthang la il shudaian.

4. Anung îlthing-as gum gin tlishgūdangung lth gudung-gung, waigien hin Pilate 
liplēt un ga itlagadasgai isgien, tl shkulas ishin, shudaian.

5. Waigien wau telg tl gushūīdan, Tl haada gin dāung-a il kil-isdagung-gung, waigien Judæa tligai ashkwau-hun gut gin tla il shkotadagung-gung, waigien Galilee tligai gu il istlagang-gun sta, adshi tligai un ishīn il katlang, waigien hin tl shouon.

6. Waigien tlistleuon Pilate gudang-andlu, il Galilee haadāgai da tla alth il kianang-an.

7. Waigien tlistleuon Herod gia tligai sta il ijan un il ūnshidalandlu, Herod à la il kilkaidan, la ishin waudlu Jerusalem gu ijan altha.

8. Waigien tlistleuon Herod Jesus kangandlu, il gudung-ai lā-euon-an ; il kang-gai da la il gudung-hunsgadan altha, la alth gialthing-e il gudung-gang-an altha; waigien gin unkuldung-a il isdas lang-a kang-gai da il gudang-an.

9. Waigien gin kwon da lia alth il kianangan ; waigien gum hanglthang la il shudang-an.

10. Waigien liplēt un ga itlagadasgai isgien, ga tukālung-lēgasgai ishin tljūgiang-an, waigien la hanltha tl gushu-euon-an.

11. Waigien Herod gyang soldiers-gai dungalth la alth nang-asilang-an gien, la alth il kilth-nang-an, waigien gin giandalthing-e lā ai la il kudsadaian gien, Pilate à silthgang la il kaidaian. 
12. Waigien gai sing-ai gut Herod isgien Pilate ishin gut un gudang-ai ligalan; wau kōnast gu telg il keng-ūgang-an altha.

13. Waigien Pilate liplet un ga itlagadasgai isgien, ga itlagadasgai ishin, tl haada ishin un ai-ang-an gien, hin tla il shudaian,

14. Anung ilthing-as di $\bar{a}$ dalung isdagun gien, tl haada gin dāung-a il kil-isdang dalung shugun; waigien gwokeng, dalung hang-gu la lth ging-kilisalung-gun, waigien la hanltha gin dāung-a dalung shudas gum il isdang-an un di ünshidulgun ;

15. Herod ishin ging-an shugung; itil $\bar{a}$ housen silthgang la il kilkaidan altha ; waigien gwokeng, gin agunan la tl tīi-althing-e las gum il isdang-an.

16. Althji alth la lth shkiduns gien, la lth kaidāashang, waigien hin tla il shudaian.

18. Anung ilthing-as lth dang, waigien Barabbas tl lth itil un kāgwulthda, waigien gudung-althan hin kwunan tl shouon;

19. Althunis ilnagai gu gudan lthkwīda tloulthaian gien, ishin tla il tldaian tāa kechitnai ai tl isdaian.

20. Waigien housen Pilate tla à gushou-on, Jesus kāgwulthdie da il gudang-an altha;

21. La lth jidūdlishgut, la lth jidūdlishgut, waigien hin kwunan tl shouon.

22. Gushintlou, gūsh dāung-a ū anung îlthing-as isdā-üchang? Gin agunan la tl tîie las HA. 
gum lth këi-ung-gun; althji alth la lth shkiduns gien, la lth kāgwulth-dāashang, waigien hin tla il shuda-lthonalan.

23. Waigien wau telg kwunan tl kilthgūlaian gien la tl tiie da tl kianang-an. Waigien tl kilth agunan tla gwĩ käalan.

24. Waigien gin da tl kianang-an ging-an tl ūgai un Pilate tuking-gogang-an.

25. Waigien gudan lthkwīda nung tloulthaian gien, tl tldaian tāa kechitnagai ai tl isdaian il kagwulthdaian, Jesus tl ginang-an la tl isdie un tla ga il isdaian.

26. Waigien tlistleuon la tl alkaidandlu, Simon, Cyrene haadas, ilnagai kyāgūst katlagan tl gitsgilthdaian gien, shkau-amagai la tl shkeulthgaidaian, Jesus dlā il shkeulth-gagundalai una.

27. Waigien tl shkūl-euon la dlā istalan, waigien tl jāada ishin saigaian gien, la silth tl shouon.

28. Waigien Jesus tla gwī kè-aian gien, hin il shouon, Jerusalem gūjang-alung isis, gum lth di kalthshint saigang-ū ; kalthshintgang isgien, gitalung kalthshint ishin lth saigū.

29. Ga kekulasgai isgien, gum ga kaiwonsgai ishin, waigien gum kun-ung alth ga tutlindansgai ishin, lagung, hin tl shū-ases shantlanai isangkasas altha.

30. Itil gwi lth tl-dulthdi, hin wau dlu 
tldāwe tl shūdiids gien, Itil lth sal, hin ishin kēi-âwons tl shudīidshang.

31. Khait hēnung-a hin tl isdasdlu, gushinu khait yhilgala tl isda-tljū-ashang? hin tla il shudaian.

32. Waigien gin dāung-a isdālē-alung stung la alth tl al-istalan, la tl tl-dāwe una.

33. Waigien tlistleuon Kuts Shkwuds hin tliga kyāgan un tl istlagandlu, la tl jitūdlishgadan, waigien gin dāung-a isdalēalung ishin tl jitūhashgadan, nung swon sōolgūst tl isdaian gien, nung swon ishin stlan-gūst $\mathrm{tl}$ isdaian.

34. Aung-a, tla kalthshint lth gudung; gum tliku tl wos un ung-a tl ūnshidans altha, waigien hin Jesus shouon. Waigien gin il giandaian gūdī tl tläadīgan gien, lots tl isdaian.

35. Waigien tl haada tljūgiang-an gien, la tl khä-ü-lang-an. Waigien ga itlagadasgai ishin la alth kilthnang-an, Tl kulat il kogundagini; akwon lth agung il kogundadi, Shanung-itlagadas gia Christgai il isis gien, nung il gwülāgan ishin il isisdlu-a, waigien hin tl shouon.

36. Waigien ga soldiergasgai ishin la alth shou-nang-an, waigien la un tl istlagan gien, vinegar la ga tl king-āwon,

37. Jews haade un nung king-gas dung isisdlu lth, agung kogundi, waigien hin tl shonon.

38. UNIS JEWS HAADE UN NUNG 
KING-GAS IJUNG, waigien hin la sha-it gin kālung-ăgan.

39. Waigien gin dāung-a isdalē-alung swon dliiwaian la alth kilthnang-an, Gum gu nung Christgas dung isang-ūsh ? agung lth kogundi, waigien itil ishin, waigien hin il shouon.

40. Nung swon tlou hanglthang shouon gien, la il stidaian, Gum gu Shanung-itlagadas ga hun dung lthwaugang-üsh, la ging-an dung tlū-unshkotias altha?

41. Itil $\bar{u}$ tlūi-un-shkotiāgai lāgung; gin talung isdagini itil ga shougas altha; unis tlou gum gin dāung-a isdang-gun, waigien hin il shouon.

42. Jesus, di ai lth kēidung, tlistleuon kingdomgai dung-alth ung-a dung katlasdlu-a, waigien hin il shouon.

43. Yenki dung ga lth shugung, Aiat, Paradise gu di alth dung isisang, waigien hin la il shudaian.

44. Waigien waudla ashkoshgît tlū-unilthēlan, waigien ashkoshgīt tlāalth-swonshung-gūelē keu tliga ashkwau-hun gut algāgan,

45. Jūyē otgagai gū-êlan altha; waigien templegai giang-u alth roomgagan gũdista āgitajan.

46. Waigien tlistleuon Jesus kwūnan sailthaiandlu, Aung-a, dung stlai ai althandang lth isdang, hin il shouon; waigien hin il shugigandlu, il althandai la sta ijan. 
47. Waigien tlistleuon nung centurion-gas tliku gin edan kang-andlu, Shanung-itlagadas $\bar{a}$ il kil-lāgan, Yenki unis nung îlthing-a lā ijan, waigien hin il shouon.

48. Waigien ga shkūlasgai adshi kang-gai un gūdā ijan wautliwon tliku gin ets kangandlu, kun-ung tl shkwūdang-an gien, tl stëlan.

49. Waigien la un ga ūnshidan-gai wautliwon isgien, Galilee sta tl jāadas la dlā istalan ishin, wochgwau tljūgiang-an gien, adshi tl keng-gang-an.

50. Waigien nung îlthing-a Joseph hin kyāgan council haadāgan, waigien nung ilthing-a lā il ijan gien, yenki gin et swonan ishin il isdagang-an.

51. (Althunis gin tl kilth-elthgiggan isgien, gin tl isdaian ishin gūdāung-aian;) Jews haade gia ilnagai swon, Arimathæa hin kyāgan sta il ijan, waigien Shanung-itlagadas gia kingdomgai keu il khatsūgang-an ;

52. Unis Pilate à kāgan gien, Jesus kōt il ginang-an.

53. Waigien het ga la il isdaian gien, la odō gīang- $\bar{u}$ il isdaian, waigien shalthung-nai tish ai jit-helthiladsaian ai la il isdaian, waigien gum hawun à nung kōt isang-an.

54. Waudlu gin tla-elthgi shantlanai ijan, waigien sabbath shantlanai dōng-ēlan.

55. Waigien tl jāada Galilee sta la alth ijan tla dlā istalan, waigien shalthung-nagai

HA.

K 3 
isgien, tliku il kōt tl dla-āwon ishin il kengāwon.

56. Waigien il stīlthāwon gien, gin shgūnūla isgien, hyil tō-a ishin, il tloulthāwon. Waigien sabbath shantlanai ai, tliku king-gogung-ai shus ging-an, agung il shandsū dāwon.

\section{Chapter 24.} WIGIEN sabbath shantlanai sta shantlan
tlagang-andlu, sing-ai un shalthungnagai un il istla-âwon gien, gin shgūnūla il tloulthāwon il giālatla-āwon.

2. Waigien shalthung-nagai sta kwau-e shkōkēstliāgan il keng-āwon.

3. Waigien ai il isdsāwon gien, gum Shalana Jesus kōt il keng-ang-āwon.

4. Waigien hawun odō il kwūlthilthūgundān, tl ilthansida stung gin otga-euon gianda la kwulth tljūgi-ai-āwon;

5. Waigien il lthwaugulth-ā won gien, tligai ing-gu il tīgūstlū-âwondlu, hin la il shudāwon, Gushintlou ga kotultbgiasgai shu-wīt nung bēnung-as da dalung dì-ing-gung?

6. Gum adlun il isang-gung, il kālthūgun altha; Galilee gu hawun il ijandlu, tliku dalung $\bar{a}$ il gushouon $\bar{a}$ lth gūshgadū,

7. Nung haada Git tl haada dāung-a stlai ai tl isdas gien, ishin la tl jitūdlishguts gien, shantlan lthonultbīlthsdlu housen il kālthūe 
lāgung, hin il shouon, waigien hin la il shudāwon.

8. Waigien tliku il shouon ai il kēidāwon,

9. Waigien shalthung-nagai sta il stīlthāwon gien, ga tlāalth waugu swonshunsgai ga isgien, tl kulat wautliwon ga ishin, adshi wautliwon il shudāwon.

10. Waigien Mary Magdalene isgien, Joanna ishin, Mary James ou jjan ishin, tl jāada kulat la alth isãwon ishin, a gin isis ga apostlegasgai ga shudaian

11. Waigien adshi gushue ging-gang-an gushou èduns ging-an la un edä̀won; waigien gum il yetdang-āwon.

12. Peter tlou giāgan gien, shalthung-nagai $\bar{a}$ il odan; waigien hēt il gūjalan gien ai il kēidsai-an, waigien giang-we swonan wau isī il kang-an; waigien gai sta nai à ung-a il kaidan gien tliku gin edan un il kuldung-āgan.

13. Waigien tl stung $\bar{u}$ gai sing-ai ai lana Emmaus hin kyāgan à istīidan; waigien althji ilnagai furlong thäale tlu-unilth Jerusalem sta jīng-āgan.

14. Waigien tliku gin edan wautliwon edë il kāūgāwon.

15. Waigien hawun edē il kāūgūu-wos gien, ishin il whūndūgundan, Jesus lit un katlaàwon gien la alth il kāa-âwon.

16. Waigien gum agung la il shkotangàwon, gum la un il ūnshidang-awe una. 
17. Gūshū dalung istals in ā gut ga dalung kāūgang? waigien hin la il shudāwon. Waigien il tljūgia-āwon gien, il gudung-ai stihunggung-āwon.

18. Waigien nung swon, Cleopas hin kỹāgan, hin hanglthang il shudaian, Jerusalem gu dung nas-kianan gu, tliku itan gu gin ëdun gum un dung ūnshidang-ūsh? hin il shouon.

19. Gūshū ? waigien hin la il shudāwon. Jesus Nazareth haadas alth gialthing-e-a, Shanung-itlagadas hang-gu isgien, tl haada wautliwon hang-gu ishin, gin isdie un isgien, gin shudie un ishin, nung prophetga dugwia il ijun;

20. Waigien liplēt un ga itlagadasgai isgien, itil un ga itlagadargai ishin nung governorgas il kotulai lang shougai un la à la tl isdagun waigien la tl jitūdlishgīdun.

21. Waigien Israel haade nung kogundaung-kashagun il ising talung gudung-gun. $\bar{A}$ ng, waigien adshi wautliwon îlā, aiat adshi ijun sta shin lthonulgung.

22. Waigien itil tō-alung jāadas ilthi itil kil-kuldung-gun, sing-ai un shalthung-nagai gu il isāwon altha;

23. Waigien tlistleuon gum il kōt il kengung-āwondlu, il istlā-ūgun, waigien angels ishin il keng-äwon gien, il hēnung-ang la ga il shu-āwon il shū-ūgun.

24. Waigien itil alth ga ijun-gai ilthi shalth- 
ung-nagai $\bar{a}$ istīidan, waigien tliku tl jāadas shugun ging-an etsi il keng-āwon; la tlou gum il keng-ung-āwon, waigien hin la il shudāwon.

25. Tl ilthansida kōnung-a dalung isis gien, tliku ga prophetgas-gai gin shudaian wautliwon yetdie un dalung gūt-hōlang-gung!

26. Gum gu Christ a gin shing-idsas ānduns gien, gyang unyāgudung-gai ai il kudsie lāung-ūjā? waigien hin la il shudāwon.

27. Waigien Moses gia kōgīnē à isgien, ga prophetgas wautliwon gia kōgīnē à ishin, biblegai $\bar{a}$ kōgīn kulat wautliwon $\bar{a}$ ishin, la edē gin kālung-as la ga il shudāwon.

28. Waigien lana à il istalāron un il dôngelthāwon; waigien tl kā-swonung-ases ging-an il edan.

29. Waigien la os il gudung-ầ won gien, Itil $\mathrm{kwulth}$ lth is, sing-idalths altha, waigien hawìdan algēlthshang, hin il shu-āwon. Waigien la kwulth il isawe un il kudsaian.

30. Waigien la alth il lthdanū-ūwe un il kou-ondlu, shibligai il isdaian gien, sing il killägan, waigien ai il ginanang-an gien, la ga il isdāwon.

31. Waigien il hung-i helthilthgāwon gien, la un il ūnshidāwon; waigien umlthīstan gum il kang-gang-an.

32. Hawun keu-e gut talung gundalgnu gien, bible kālung-e itil un wausta il da-ostla- 
gundlu, gum gu itil gūdung-ai kīn-euon-ungūja? waigien hin gu il shudāwon.

33. Waigien gai ashkoshgadai gut il tljūgiāwon gien, Jerusalem gwī il stīlthāwon, waigien ga tlaalth waugu swonshunsgai isgien, la alth ga isāwon-gai ishin gudung-althan gudā ijan il keng-āwon,

34. Yenkien Shalana kotal sta kalthāwon gien, Simon agung il kendaian, waigien hin il shu-āwon.

35. Waigien keu-e gut il istalāwondlu tliku gin edan alth isgien, tliku shibligai ai il ginanang-an alth la un il ūnshidāwon alth ishin, tla ga il gialthindāwon.

36. Waigien hawun adshi il shūdūgundān, la yākosīa la kodan gia-āwon, Akwon lth gudung-ung dlaidū, waigien hin la il shudāwon.

37. Waigien il gudung-ai lthkwīdāwon gien, il lthwaugāwon, waigien hants il keng-wong il gudung-āwon.

38. Waigien hin la il shudāwon, Gushintlou dalung gudung-ai lthkwīdang? gushintlou dalung kwulthilth-euon-gung?

39. Di stlai lth keng-ū, di stai ishin, lthā- $\bar{u}$ ijung; di lth tlikūdung-ū ang-a di keng-ū ; tliku di ets dalung kens ging-an, gum hants kyā-uns gien, ishin gum shkwüjāgung-uns altha, hin la il shudāwon.

40. Waigien tlistleuon hin il shouondlu, stlang isg en, stang ishin, la il kendāwon. 
41. Waigien il gudung-ai lā-wos tista gum hawun il yetdang-wos gien, ishin il kuldung-āūgundān, Gum gu gin talthing-e dalung dāung? hin la il shudāwon.

42. Waigien shkolthung gittsā la ga il isdāwon.

43. Waigien il isdaian gien, la hang-gu il tā̄āwon.

44. Waigien hin la il shudāwon, Hawun dalung kwulth lth ijundlu, tliku dalung ga lth shugun $\overline{\mathrm{u}}$ aे ijung; Moses gia king-gogung-ai $\bar{a}$ isgien, ga prophetgasgai gia kōgīnē $\bar{a}$ ishin, psalm kōgīnē à ishin, tliku di edē gin kālungas wautliwon wai ging-an etgai lagung, waigien hin la il shudāwon.

45. Itan il gudung-ai il helthilthgadāwon, tliku bible kālung-ai ets un il ūnshidawē una;

46. Waigien hin la il shudāwon, Nung Christgas wauguns gien, shin lthōnulth-illthsdlu housen kotal sta il kālthue lagung;

47. Waigien il kyē agunan, gudung-ung stîlthda isgien, gin dā-ung-a da kēshgade alth ishin, gwaigang-ai wautliwon geu gu tl gushue lagung, waigien Jerusalem gu tl shuda-tlagangai lagung, hin kālung-āgung.

48. Dalung $\bar{u}$ adshi un ūnshitlēalung ijung.

49. Waigien di Aung gin shudagun dalung: à lth hundsūdāashang; waigien sha-tligai sta dugwia is dalung giandile keu lth ilnagai gu iswong, waigien hin la il shudāwon. 
50. Waigien Bethany hanltha il istlāawe keu la il alistalāwon; waigien shi ga stlang il isdaian gien, gushou lā la ā il shudāwon.

51. Waigien hawun gushou lā la à il shūdūgundān, la sta il isāwon gien, shi ga il kulthaian.

52. Waigien la à singlthgang il gushu-āwon gien, gudan lā-euon dung-alth Jerusalem gwī il stîlthāwon;

53. Waigien temple-gai $\bar{a}$ il isgiūgang-an gien, Shanung-itlagadas ā il kil-lā-ūgang-an. 





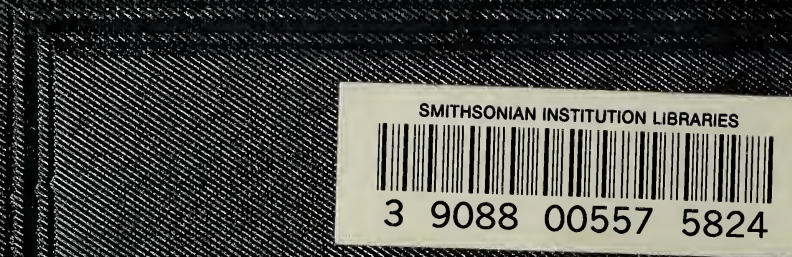

